A\&A 659, A145 (2022)

https://doi.org/10.1051/0004-6361/202142648

(c) ESO 2022

\title{
A WISE view on extreme AGB stars ${ }^{\star, \star \star}$
}

\author{
M. A. T. Groenewegen (
}

Koninklijke Sterrenwacht van België, Ringlaan 3, 1180 Brussels, Belgium

e-mail: martin.groenewegen@oma.be

Received 12 November 2021 / Accepted 31 December 2021

\begin{abstract}
Context. Variability is a key property of stars on the asymptotic giant branch (AGB). Their pulsation period is related to the luminosity and mass-loss rate (MLR) of the star. Long-period variables (LPVs) and Mira variables are the most prominent of all types of variability of evolved stars. However, the reddest, most obscured AGB stars are too faint in the optical and have eluded large variability surveys.

Aims. Our goal is to obtain a sample of LPVs with large MLRs by analysing WISE $W 1$ and $W 2$ light curves (LCs) for about 2000 sources, photometrically selected to include known C-stars with the $11.3 \mu \mathrm{m}$ silicon carbide dust feature in absorption, and Galactic O-stars with periods longer than 1000 days.

Methods. Epoch photometry was retrieved from the AllWISE and NEOWISE database and fitted with a sinus curve. Photometry from other variability surveys was also downloaded and fitted. For a subset of 316 of the reddest stars, spectral energy distributions (SEDs) were constructed, and, together with mid-infrared (MIR) spectra when available, fitted with a dust radiative transfer programme in order to derive MLRs.

Results. WISE based LCs and fits to the data are presented for all stars. Periods from the literature and periods from refitting other literature data are presented. The results of the spatial correlation with several (IR) databases is presented. About one-third of the sources are found to be not real, but it appears that these cannot be easily filtered out by using WISE flags. Some are clones of extremely bright sources, and in some cases the LCs show the known pulsation period. Inspired by a recent paper, a number of nonvariable OH/IRs are identified. Based on a selection on amplitude, a sample of about 750 (candidate) LPVs is selected of which 145 have periods $>1000$ days, many of them being new. For the subset of the stars with the colours of C-rich extremely red objects (EROs) the fitting of the SEDs (and available MIR spectra) separates them into C- and O-rich objects. Interestingly, the fitting of MIR spectra of mass-losing C-stars is shown to be a powerful tracer of interstellar reddening when $A_{V} \gtrsim 2$ mag. The number of Galactic EROs appears to be complete up to about $5 \mathrm{kpc}$ and a total dust return rate in the solar neighbourhood for this class is determined. In the LMC 12 additional EROs are identified. Although this represents only about $0.15 \%$ of the total known LMC C-star population adding their MLRs increases the previously estimated dust return by $8 \%$. Based on the EROs in the Magellanic Clouds, a bolometric period luminosity is derived. It is pointed out that due to their faintness, EROs and similar O-rich objects are ideal targets for a NIR version of Gaia to obtain distances, observing in the $K$-band or, even more efficiently, in the $L$-band.
\end{abstract}

Key words. stars: variables: general - infrared: stars - stars: AGB and post-AGB

\section{Introduction}

At the end of their lives almost all low- and intermediatemass stars (with initial masses from $\sim 0.9$ to $\sim 10 M_{\odot}$ ) will go through the (super)-asymptotic giant branch ((S-)AGB) phase. They end up as $\sim 0.55-1.4 M_{\odot}$ white dwarfs which implies that a large fraction of the initial mass of a star is returned to the interstellar medium (ISM). Pulsation is an important characteristic of AGB stars, and they are typically divided into stars with small amplitudes (the semi-regular variables, SRVs) and the large-amplitude Mira variables. The term long-period variable (LPV) is now commonly used for a pulsating AGB star, regardless of pulsation amplitude. The most promising mechanisms to explain wind driving are pulsation-induced shock waves and radiation pressure on dust, especially regarding the more evolved AGB stars with low effective temperatures, large pulsa-

* Full Tables 2, A.1-A.6, and C.1 are only available at the CDS via anonymous ftp to cdsarc.u-strasbg. fr (130.79.128.5) or via http://cdsarc.u-strasbg.fr/viz-bin/cat/J/A+A/659/A145

$\star \star$ Figures B.1-B.6, and C.2 are available at https ://doi .org/10. $5281 /$ zenodo. 5825878 tion amplitudes, and high mass-loss rates (MLRs; see the review by Höfner \& Olofsson 2018).

Analysis of the MLRs of essentially complete samples of AGB stars in the Magellanic Clouds (MCs) has shown that the gas and dust return to the ISM is dominated by a small percentage of stars with the highest MLRs (e.g. Matsuura et al. 2009; Boyer et al. 2012; Nanni et al. 2019, and references therein). These stars are often characterised by the longest pulsation periods.

Current surveys in the optical domain (including OGLE and Gaia) will, however, miss the reddest, most obscured AGB stars. At the very end of the AGB the (dust) MLR may become so high that the object becomes very faint, beyond the OGLE $I$-band detection limit of about $21 \mathrm{mag}$ or the Gaia G-band limit of about $21.5 \mathrm{mag}$. The dust grains in the circumstellar envelope (CSE) scatter and absorb the emission in the optical to re-emit it in the NIR and mid-infrared (MIR), where they become bright sources. These stars are known to exist in the MCs. They were initially selected and identified as having Infrared Astronomical Satellite (IRAS) colours similar to obscured AGB stars in our Galaxy, and later on photometric and spectroscopic observations with the Spitzer Space Telescope 
(SST, Werner et al. 2004) confirmed this and added additional examples of this class of extreme mass-losing objects, mostly being carbon-rich AGB stars (Gruendl et al. 2008; Sloan et al. 2016; Groenewegen \& Sloan 2018).

In an earlier related work, Groenewegen et al. (2020) presented a sample of 217 likely LPVs in the MCs. This paper investigated the variability of 1299 objects in the $K$-band, based on VISTA Magellanic Cloud (VMC) survey data (Cioni et al. 2011), supplemented with literature data. The aim of that paper was also to find red AGB stars with long periods, although potentially not as red as the sources studied here as the very reddest sources will also be faint or invisible even in the $K$ band. Although the VMC data are of high quality the sampling is not optimal for detecting LPVs (typically 15 data points spread over 6 months ordinarily). Although $K$-band data from the literature was added (e.g. 2MASS Cutri et al. 2003 , 2MASS 6X Cutri et al. 2012, IRSF Kato et al. 2007, DENIS DENIS Consortium 2005, as well as the pioneering monitoring works of Wood et al. 1992; Wood 1998; Whitelock et al. 2003), in some cases, no unique period could be derived and several periods could fit the $K$-band data.

The Wide-field Infrared Survey Explorer (WISE; Wright et al. 2010) and the Near-Earth Object WISE (NEOWISE) and NEOWISE Reactivation mission (Mainzer et al. 2011, 2014) are ideal surveys to study LPVs. The total time span covered is about $9 \mathrm{yr}$ which covers two or more pulsation cycles even for extremely long periods. Other advantages are that they survey at wavelengths where the reddest objects are the brightest, and they survey the entire sky.

Previous studies already explored the time variability offered by the WISE mission. Chen et al. (2018) presented a catalogue of $\sim 50000$ periodic variables with periods shorter than 10 days, Petrosky et al. (2021) presented a similar catalogue of $\sim 63500$ periodic variables with periods shorter than 10 days (using different criteria), while Uchiyama \& Ichikawa (2019) studied the MIR variability in massive young stellar objects (YSOs).

The outline of the paper is as follows. Section 2 introduces the sample of known very red C- and O-rich AGB stars that will serve as templates to select candidates based on photometric selection criteria using AllWISE data. Section 3 describes the selection of the time series data both from the WISE mission and other literature data, and the analysis and fitting of the time series data. Section 4 outlines the results of an extensive literature study into the classification of the objects. Section 5 briefly describes the various tables that contain the results of the literature search and the period analysis. Section 6 discusses these results by addressing various topics in more detail, including the discovery of new LPVs with periods over 1000 days and new AGB stars with extremely large MLRs.

\section{The template sample and source selection}

This section describes the template sources of very long-period variables and very red sources that were used to create a WISE colour-selected sample of candidate very evolved AGB stars.

\subsection{Carbon-rich $A G B$ stars}

The term extreme AGB star is not well defined. It was probably first used by Volk et al. (1992) in connection with carbon-rich AGB stars (hereafter C-stars). Their investigation was spurred by the fact that previous surveys in the infrared, such as the Two-micron sky survey (Neugebauer \& Leighton 1969) and the Air Force Geophysics Lab (AFGL, Price \& Walker 1976) sur- vey discovered C-stars with unusually thick dust shells, such as IRC +10216 (CW Leo) or AFGL 3068. They selected a group of 31 stars based on certain spectral characteristics observed in 8-23 $\mu \mathrm{m}$ Low Resolution Spectrograph (LRS) data taken during the IRAS mission. Independently, Groenewegen et al. (1992) listed eight sources (out of 109, their 'group V' class) in their flux-limited (IRAS $S_{12}>100 \mathrm{Jy}$ ) sample of C-stars with very similar properties to those in Volk et al. (1992) based on the IRAS colour-colour diagram and LRS types. Later, Speck et al. (2009) studied ten of these sources (one new) using superior Infrared Space Observatory (ISO) Short Wavelength Spectrometer (SWS) data. Many of these sources displayed the silicon carbide ( $\mathrm{SiC}) 11.3 \mu \mathrm{m}$ dust feature in absorption, indicating a very large optical depth as the feature is normally seen in emission in C-stars. The sample of seven known C-stars with SiC in absorption is listed in Table 1, together with the WISE magnitude, the error in the magnitude, and the signal-to-noise (some being negative) in the four bands of WISE ( $W 1$ at $3.4 \mu \mathrm{m}, W 2$ at $4.6 \mu \mathrm{m}$, $W 3$ at $12 \mu \mathrm{m}$, and $W 4$ at $22 \mu \mathrm{m})$.

Then, Gruendl et al. (2008) discussed a dozen sources in the direction of the Large Magellanic Cloud (LMC) characterised by extremely red MIR colours ([4.5]-[8.0] > 4.0) based on SST colours and spectral energy distributions (SEDs), peaking between 8 and $24 \mu \mathrm{m}$. Seven of those show a flat red continuum or $\mathrm{SiC}$ in absorption based on Infrared Spectrograph (IRS; Houck et al. 2004) data. They introduced the term extremely red objects (EROs). They did not discuss any link with the known similar objects in the Milky Way.

Table 1 lists the properties of those seven sources together with four other LMC sources with $\mathrm{SiC}$ in absorption based on other IRS programmes, see Sloan et al. (2016) and Groenewegen \& Sloan (2018). Interestingly, no known EROs exist in the SMC. Ventura et al. (2016) explained the fact that the reddest $\mathrm{C}$-stars in the $\mathrm{LMC}$ are redder than the reddest $\mathrm{C}$ stars in the Small Magellanic Cloud (SMC), which is related to a difference in initial mass $\left(2.5-3 M_{\odot}\right.$, respectively, $\left.\sim 1.5 M_{\odot}\right)$, consistent with the difference in star formation histories between the two galaxies.

To complete the description of the terminology, the term extreme AGB stars (often designated X-AGB stars or X-stars) is also used in the literature based on photometric criteria (and it can refer to C-stars or oxygen-rich AGB stars (hereafter Ostars)), for example Blum et al. (2006) who used a limit of $J-[3.6]>3.1$. As x-AGB stars can be invisible in the $J$-band, other criteria have been adopted, for example [3.6]-[8.0] $>0.8$ (Boyer et al. 2011) or [3.6]-[4.5]>0.1 (Boyer et al. 2015). As discussed in Sloan et al. (2016), this terminology is something of a misnomer as sources with such colours produce an appreciable amount of dust, but this is a common phenomenon as stars evolve on the AGB, and they are not 'extreme' in that sense. The $\mathrm{C}$-stars with a red flat continua or $\mathrm{SiC}$ in absorption are a subset of x-AGB stars, and they represent the reddest colours, for example [3.6]-[4.5] $\gtrsim 1.5$ (Sloan et al. 2016).

\subsection{Oxygen-rich $A G B$ stars}

As a class, the $\mathrm{OH} / \mathrm{IR}$ stars (see Hyland 1974 for an early review) come closest to being called extreme O-stars, as they can be very red $(K-L>7$, Jones et al. 1982) and are recognised as the O-stars with the largest MLRs (Herman \& Habing 1985). In $\mathrm{C}$-stars $\mathrm{SiC}$ is a minor dust species compared to amorphous carbon and so for the $11.3 \mu \mathrm{m}$ to go into absorption very high dust densities are required. On the other hand, silicates are the dominant species in the CSEs around O-stars and therefore stars 
Table 1. Template sample of extreme AGB stars.

\begin{tabular}{|c|c|c|c|c|c|c|c|c|c|c|c|c|}
\hline Name & $\begin{array}{r}W 1 \\
(\mathrm{mag})\end{array}$ & $\begin{array}{l}\text { Error } \\
(\mathrm{mag})\end{array}$ & $S / N$ & $\begin{array}{r}W 2 \\
(\mathrm{mag})\end{array}$ & $\begin{array}{r}\text { Error } \\
(\mathrm{mag})\end{array}$ & $S / N$ & $\begin{array}{r}W 3 \\
\text { (mag) }\end{array}$ & $\begin{array}{r}\text { Error } \\
(\mathrm{mag})\end{array}$ & $S / N$ & $\begin{array}{r}W 4 \\
(\mathrm{mag})\end{array}$ & $\begin{array}{r}\text { Error } \\
\text { (mag) }\end{array}$ & $S / N$ \\
\hline \multicolumn{13}{|c|}{ Known EROs in the Galaxy } \\
\hline AFGL 190 & 7.445 & 0.025 & 42.7 & 3.264 & 0.255 & 4.3 & -1.449 & 0.346 & 3.1 & -3.137 & 0.002 & 575. \\
\hline AFGL 3068 & 4.689 & 0.288 & 3.8 & -0.085 & - & 0.9 & -3.063 & - & 0.6 & -3.975 & 0.002 & 625. \\
\hline AFGL 3116 & -0.480 & - & 0.4 & 1.721 & - & -9.3 & -2.966 & - & 1.3 & -3.493 & 0.002 & 703. \\
\hline IRAS 08171-2134 & 7.340 & 0.053 & 20.5 & 3.795 & 0.313 & 3.5 & -0.755 & 0.395 & 2.8 & -2.766 & 0.001 & 816. \\
\hline IRAS $19075+0921$ & 6.802 & 0.064 & 16.9 & 3.044 & 0.416 & 2.6 & -1.165 & 0.354 & 3.1 & -3.105 & 0.002 & 462. \\
\hline IRAS $15471-5644$ & 6.063 & 0.097 & 11.2 & 1.907 & - & 1.9 & -1.333 & 0.386 & 2.8 & -3.191 & 0.001 & 865. \\
\hline IRAS $21318+5631$ & 6.276 & 0.037 & 29.3 & 1.707 & - & 1.8 & -1.859 & 0.349 & 3.1 & -3.263 & 0.002 & 580. \\
\hline \multicolumn{13}{|c|}{ Known EROs in the LMC } \\
\hline ERO 0502315 & 17.233 & 0.067 & 16.2 & 12.693 & 0.021 & 51.7 & 5.553 & 0.012 & 73.7 & 3.131 & 0.015 & 72.7 \\
\hline ERO 0504056 & 18.692 & - & 1.9 & 13.058 & 0.023 & 47.0 & 5.919 & 0.014 & 77.5 & 3.653 & 0.014 & 77.9 \\
\hline ERO 0518117 & 14.752 & 0.026 & 42.6 & 11.481 & 0.020 & 53.9 & 5.366 & 0.014 & 76.2 & 2.887 & 0.0 & 98.8 \\
\hline ERO 0518484 & 16.293 & 0.476 & 2.3 & & 0.036 & & 5.597 & 0.014 & 79.2 & 3.406 & & 55.0 \\
\hline ERO 0525406 & 16.831 & - & -0.5 & 13.427 & 0.040 & 27.1 & 6.136 & 0.010 & 105. & 3.855 & 0.020 & 54.5 \\
\hline ERO 0529379 & 13.672 & 0.023 & 47.0 & 10.259 & 0.020 & 54.4 & 5.491 & 0.014 & 76.0 & 3.649 & 0.017 & 64.2 \\
\hline ERO 0550261 & 14.939 & 0.031 & 35.3 & 10.787 & 0.020 & 54.9 & 4.848 & 0.015 & 74.6 & 2.793 & 0.012 & 89.6 \\
\hline IRAS 05133-6937 & 19.082 & - & -48 & 14.289 & 0.042 & 26.0 & 5.955 & 0.018 & 59.7 & 3.444 & 0.023 & 47.7 \\
\hline IRAS 05315-7145 & 13.910 & 0.026 & 41.2 & 11.992 & 0.022 & 49.9 & 5.779 & 0.014 & 76.2 & 2.939 & 0.0 & 82.5 \\
\hline IRAS 05495-7034 & 15.733 & 0.030 & 36.1 & 13.558 & 0.024 & 45.7 & 5.864 & 0.012 & 87.7 & 2.608 & 0.009 & 116. \\
\hline IRAS $05568-6753$ & 11.113 & 0.023 & 47.6 & 8.238 & 0.020 & 53.1 & 4.316 & 0.014 & 77.0 & 2.787 & 0.011 & 95.5 \\
\hline \multicolumn{13}{|c|}{ Known Galactic O-stars with $P>1000$ days (Menzies et al. 2019) } \\
\hline V1360 Aql, OH 30.7+0.4 & 5.360 & 0.075 & 14.5 & 1.309 & - & 1.4 & 0.237 & 0.423 & 2.6 & & 0.009 & 119.6 \\
\hline V1362 Aql, OH 30.1-0.7 & 6.935 & 0.143 & 7.6 & 1.625 & - & 1.6 & -0.659 & 0.477 & 2.3 & -3.453 & 0.006 & 188.8 \\
\hline V1363 Aql, OH 32.0-0.5 & & 0.027 & 40.1 & & 0.173 & 6.3 & 0.282 & 0.083 & 13.1 & -1.654 & & 325.9 \\
\hline V1365 Aql, OH 32.8-0.3 & 6.908 & 0.062 & 17.4 & 3.010 & 0.356 & 3.0 & 0.085 & 0.406 & 2.7 & -2.751 & 0.010 & 103.7 \\
\hline V1366 Aql, OH 39.7+0.5 & 1.522 & - & 1.2 & -1.304 & - & 0.8 & -1.603 & 0.368 & 3.0 & -3.138 & 0.003 & 345.8 \\
\hline V1368 Aql, OH 42.3-0.1 & 7.686 & 0.024 & 44.7 & 3.814 & 0.040 & 26.9 & 0.407 & 0.030 & 35.9 & -1.480 & 0.006 & 186.1 \\
\hline V669 Cas, OH $127.8-0.0$ & 3.829 & 0.334 & 3.3 & -0.393 & - & 0.8 & -1.031 & 0.311 & 3.5 & -2.948 & 0.003 & 421.2 \\
\hline OH 104.9+2.4, AFGL 2885 & 2.695 & 0.016 & 68.2 & 1.647 & 0.028 & 39.3 & -1.963 & 0.235 & 4.6 & -4.075 & 0.001 & 1601.2 \\
\hline IRAS $03293+6010$, OH $141.7+3.5$ & 4.602 & 0.215 & 5.0 & 1.675 & - & 2.0 & -0.209 & 0.380 & 2.9 & -2.347 & 0.001 & 768.4 \\
\hline IRAS 05131+4530, AFGL 712 & 3.291 & 0.527 & 2.1 & 0.190 & - & 0.8 & -0.214 & 0.359 & 3.0 & -2.307 & 0.002 & 674.7 \\
\hline IRAS 07222-2005 & 4.518 & 0.257 & 4.2 & 3.240 & 0.236 & 4.6 & 1.656 & 0.018 & 59.0 & 0.435 & 0.007 & 150.3 \\
\hline V1185 Sco, OH 357-1.3 AFGL 5379 & 4.524 & 0.275 & 4.0 & -1.250 & - & 0.5 & -3.008 & - & 1.1 & -3.513 & 0.011 & 103.1 \\
\hline V437 Sct, OH 26.5+0.6 & -0.614 & - & 0.3 & -2.449 & - & 0.5 & -2.837 & - & 1.4 & -3.879 & 0.016 & 69.2 \\
\hline V438 Sct, OH 26.2-0.6 & 4.213 & 0.273 & 4.0 & 0.749 & - & 1.2 & -0.448 & 0.345 & 3.1 & -2.539 & 0.003 & 320.2 \\
\hline V441 Sct, OH $21.5+0.5$ & 9.035 & 0.025 & 44.3 & 4.047 & 0.051 & 21.2 & 0.130 & 0.022 & 49.5 & -1.761 & 0.010 & 109.1 \\
\hline IRAS $03206+6521$, OH $138.0+7.2$ & 4.186 & 0.267 & 4.1 & 0.443 & - & 0.9 & -0.341 & 0.334 & 3.3 & -2.540 & 0.001 & 815.0 \\
\hline
\end{tabular}

with the $9.8 \mu \mathrm{m}$ silicate feature in absorption are not uncommon (although in most O-rich stars it is seen in emission). At even larger densities the silicate $18 \mu \mathrm{m}$ feature also goes into absorption, and these sources have been called extreme OH/IR stars (Justtanont et al. 2015), and $\mathrm{OH} 26.5+0.6$ is a prime example (Etoka \& Diamond 2007).

Early on it was also recognised that (extreme) OH/IR stars are associated with Mira-like large-amplitude variability with (very) long periods (Engels et al. 1983; e.g. OH 26.5+0.6 has a period of 1559 days, Suh \& Kim 2002). The template sample for extreme $\mathrm{O}$-stars that is used to define selection criteria in WISE colours is the compilation of known Galactic O-stars with periods over 1000 days from Menzies et al. (2019), and the WISE properties of these stars are listed in Table 1. Menzies et al. (2019) also lists SMC and LMC variables with periods over 1000 days. These have not been used to define WISE colour-based criteria, but are all included in the final sample (except for two supergiants).

\subsection{WISE colour selection}

The AllWISE source catalogue (Cutri 2014) contains over 747 million sources. Based on the colours and signal-to-noise ratios (SNs) in Table 1 , a query ${ }^{1}$ was run on the AllWISE source catalogue as available through the IPAC Infrared Science Archive

\footnotetext{
1 The SQL query was:

WHERE

( ( ( (w2mpro-w3mpro) $>2.2$ and (w3mpro-w4mpro) $>1.4$ and $\mathrm{w} 2 \mathrm{snr}>20$. and $\mathrm{w} 3 \mathrm{snr}>50$. and $\mathrm{w} 4 \mathrm{snr}>40$.) or

((w1mpro-w2mpro) $>2.8$ and (w2mpro-w3mpro) $>2.2$ and (w3mpro-w4mpro) $>1.4$ and w1snr $>2.0$ and $\mathrm{w} 2 \mathrm{snr}>0.75$ and $\mathrm{w} 3 \mathrm{snr}>0.5$ and $\mathrm{w} 4 \mathrm{snr}>100$.) or

$(($ w1mpro-w4mpro $)>3.0$ and $w 4 \mathrm{snr}>60))$.
} 
(IRSA) $)^{2}$ to select a sub-sample of about 60000 sources, containing all of the 34 sources in Table 1.

Figure 1 shows the colour-colour diagrams (CCDs) of that sample in WISE colours. The sources from Table 1 are plotted as red triangles. To help identify the location of (post-) AGB (P-AGB) stars in these CCDs dust radiative transfer calculations were performed with the code More of DUSTY (MoD, Groenewegen 2012), which is an extension of the radiative transfer code DUSTY (Ivezić et al. 1999). This was done by using combinations of the effective temperature and temperature at the inner dust radius of $\left(T_{\mathrm{eff}}, T_{\mathrm{inn}}\right)(2600,1000),(3300,800)$, and $(4000,400 \mathrm{~K})$, representative of late-AGB and early P-AGB evolution. This was done for $\mathrm{C}$-stars, with model atmospheres from Aringer et al. (2009) and a dust mixture of $\mathrm{SiC}$ and amorphous carbon, and O-stars, with MARCS model atmospheres (Gustafsson et al. 2008) and a dust mixture of silicate and metallic iron, for 20 optical depths at $0.55 \mu \mathrm{m}$ ranging from 0.001 to 1000 . The WISE magnitudes were calculated from the SEDs and the resulting colours were plotted using different colours and symbols (see the figure caption). As expected the sequences start at blue colours and then become increasingly red as the optical depth increases.

Based on the location of the known sources and the sequence of theoretical colours, the following further selection was applied:

$$
\begin{aligned}
& -(W 2-W 3)>-2.0 \cdot(W 1-W 2)+9.0 \\
& \quad \text { or } \\
& -(W 2-W 3)<+2.0 \cdot(W 1-W 2)-3.0
\end{aligned}
$$

and

$$
\begin{aligned}
& -(W 3-W 4)<0.333 \cdot(W 2-W 3)+1.0 \\
& \quad \text { or } \\
& -(W 2-W 3)<2.3 \text { and }(W 3-W 4)<2.8 .
\end{aligned}
$$

The sources fulfilling these conditions were plotted as small triangles in Fig. 1. To avoid cluttering in the plot, the non-selected sources (small dots) were only plotted when additional criteria were fulfilled ( $S / N>45$ in all four WISE filters). As one can notice, some known sources (big red triangles) were not selected by these conditions (they are not over plotted by a small black triangle). In most cases, this is due to the extreme brightness of these sources (e.g. OH 21.5 and AFGL 3068), corrupting their colours. These sources were added to the sample manually. As discussed below, the (NEO)WISE epoch databases often do not contain useful data for these types of very bright sources, but they often have parasitic sources for which a period can be derived. Figure 2 shows the distribution on the sky with the sources in the direction of the MCs and in the Galactic plane showing up prominently.

To this pure WISE colour-selected all-sky sample the sample of 217 likely LPVs in the MCs from Groenewegen et al. (2020) was added. As mentioned in the introduction, this sample is based on the analysis of the $K$-band from the VMC survey (Cioni et al. 2011), supplemented with literature data. In some cases no unique period could be derived and several periods could fit the $K$-band data. The WISE time series data will allow one to independently determine these periods.

The total sample for which the WISE time series will be studied is 1992 objects. It is stressed that the sample (in particular the sample of about 1750 Galactic objects) should not be considered as a complete sample. The selection on the colour and $\mathrm{SN}$ will introduce biases.

\footnotetext{
2 https://irsa.ipac.caltech.edu/Missions/wise.html
}
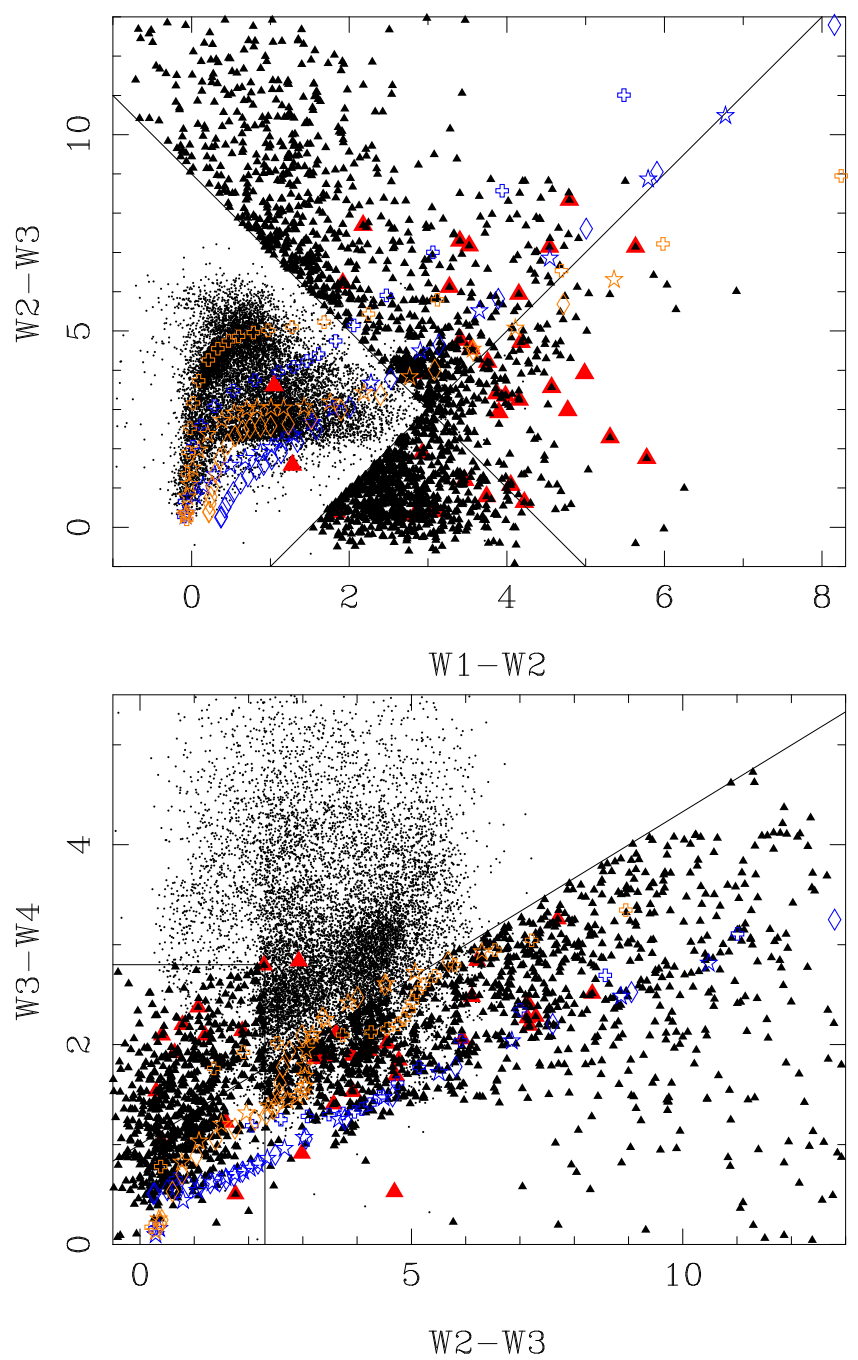

Fig. 1. $[W 2-W 3]-[W 1-W 2]$ and $[W 3-W 4]-[W 2-W 3]$ CCDs. Red triangles are the sources from the template sample in Table 1. Small black triangles are selected sources (see main text). Small dots are nonselected sources; to avoid cluttering stricter SN criteria were applied for them to be plotted (see main text). Other symbols indicate sequences of increasing MLRs (see main text). Blue colours represent C-star models while orange colours represent $\mathrm{O}$-star models for $\left(T_{\mathrm{eff}}, T_{\mathrm{inn}}\right)$ combinations of $(2600,1000),(3300,800)$, and $(4000,400 \mathrm{~K})$ in diamonds, five-pointed stars, and open plus signs, respectively.

\section{Time series data and analysis}

\subsection{WISE}

From the AllWISE multi-epoch photometry table and the NEOWISE-R single exposure source table all entries within $1^{\prime \prime}$ of the AllWISE coordinates were downloaded in the $W 1$ and $W 2$ filters with the additional constraint that the error bars on the magnitudes were $<0.25$ mag and applying the flags saa_sep $>5$ and moon_masked $=0$ (e.g. Uchiyama \& Ichikawa 2019). No other flags were applied (see Sect. 6.1).

At the bright end the WISE and NEOWISE data suffer from saturation that influences the photometry, and a correction was applied. Table 2 in Sect. II.1.c.iv.a of the NEOWISE Explanatory Supplement ${ }^{3}$ contains correction tables in the $W 1$ and $W 2$ filters for all phases of the mission. The corrections are negligible to

\footnotetext{
3 http://wise2.ipac.caltech.edu/docs/release/neowise/ expsup/sec2_1civa.html
} 


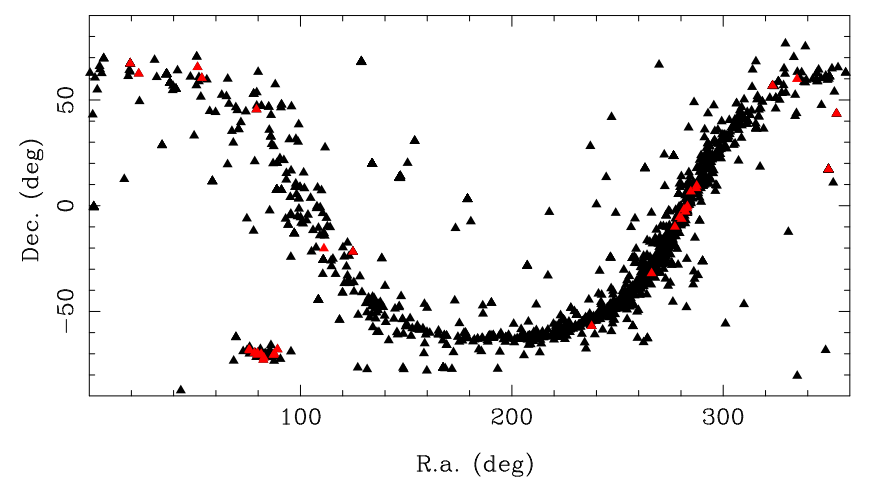

Fig. 2. Distribution on the sky of the sample. Symbols are as in Fig. 1.

small at $W 1$ and $W 2 \sim 7-8$ mag and reach almost 1.5 mag in $W 2$ in NEOWISE-R. For stars brighter than the brightest entry in the tables the corresponding correction was kept without attempting any extrapolation. The Explanatory Supplement furthermore states that even with a correction, no useful information is available for sources brighter than $W 1 \lesssim 2$ and $W 2 \lesssim 0$ mag.

\subsection{SAGE-VAR}

For stars in the direction of the MCs the WISE $W 1$ and $W 2$ data were combined with IRAC $3.6 \mu \mathrm{m}$ and IRAC $4.5 \mu \mathrm{m}$ data, respectively, from the SAGE-VAR survey (Riebel et al. 2015) that adds four epochs from the warm SST mission at 3.6 and $4.5 \mu \mathrm{m}$ for portions of the LMC and SMC. The filters of the IRAC 3.6 and 4.5 bands are similar but not identical to the WISE $W 1$ and $W 2$ filters and the transformation from Sloan et al. (2016) was used to bring the IRAC photometry to the WISE system.

\subsection{Other time series data}

As will be discussed below in detail, the literature was searched for known periods of the stars in the sample. However, next to quoting these periods, it turned out useful or even necessary to refit the original data in many cases for several reasons. In some cases the period in the literature seemed inconsistent with that expected for an LPV or inconsistent with that derived from the WISE data. In cases with multiple periods available from the literature these were sometimes inconsistent with each other. Also in the case of no or insufficient WISE data it seemed valuable to provide a pulsation period based on other data to the community. The major sources of additional time series photometry are described below.

The All-Sky Automated Survey for SuperNovae (ASAS-SN) (Shappee et al. 2014; Kochanek et al. 2017; Jayasinghe et al. 2018) identified 666502 variables. From the survey website ${ }^{4}$ the basic data of these variables was downloaded which included the coordinates and the pulsation period. A search radius of $2^{\prime \prime}$ was used to correlate it with the target list. In case the LC was refitted the $V$-band data were retrieved from this website as well.

The Asteroid Terrestrial-impact Last Alert System (ATLAS) (Tonry et al. 2018) published data on over 4.3 million candidate variable objects (Heinze et al. 2018). This dataset is available through the VizieR database ${ }^{5}$ and it was correlated with the target list using a search radius of $2^{\prime \prime}$. The ATLAS team

\footnotetext{
4 https://asas-sn.osu.edu/variables

J/AJ/156/241/table4.
}

derived periods in several different ways and two are quoted, called $f p$-LSper ('original period from fourierperiod's LombScargle periodogram') and fp-lngfitper ('final master period from the long-period Fourier fit'). The original data were retrieved via a website ${ }^{6}$ following the instructions in Appendix B in Heinze et al. (2018). ATLAS observed in two bands and the redder one (the $o$-band peaking at $0.68 \mu \mathrm{m}$ ) is used to refit the LC.

Data from the Zwicky Transient Facility (ZTF) (Masci et al. 2019; Bellm et al. 2019) was downloaded following the suggestions on their website ${ }^{7}$. This involved user-customised scripts using wget and a query to select the data ${ }^{8}$. For the sources in the target list data is available in the Sloan $g$ - and $r$-filters, and the redder one was used to refit the LC.

The VISTA Variables in the Vía Láctea (VVV) ESO Public Survey (Minniti et al. 2010) has been mapping the NIR variability in the $K_{s}$-band of the Milky Way Bulge and the adjacent southern disk. Recently, Ferreira Lopes et al. (2020) published the VVV Infrared Variability Catalogue (VIVA-I) containing data on almost 45 million variable star candidates. The catalogue contains periods based on five different methods, and also a 'best period', bestPeriod. From the VISTA Science Archive $(\mathrm{VSA})^{9}$ the basic data of the 6.7 million sources in VIVA-I with a bestPeriod $>0.5$ days were downloaded, which was then cross-matched with the target sample using a search radius of $3^{\prime \prime}$. In case the LC was refitted, the timeseries data were downloaded from a website ${ }^{10}$ using a dedicated query ${ }^{11}$ to obtain the publically available data.

The analysis of $3.6 \mathrm{yr}$ of data from the Diffuse Infrared Background Experiment (DIRBE) provided a list 597 (candidate) variables (Price et al. 2010). The data are available through Vizie $\mathrm{R}^{12}$ and this includes coordinates, mean magnitudes and errors, amplitudes, and periods in four photometric bands. A pairing radius of $8^{\prime \prime}$ was used. The VizieR table also includes a link to the time series data, which is used when the DIRBE data are refitted. In those cases the data at $4.9 \mu \mathrm{m}$ was used as they are typically the brightest for the sources in the target list.

Data from other surveys has been analysed for a handful of sources, namely the Optical Monitoring Camera (OMC) data on board INTEGRAL (Alfonso-Garzón et al. 2012; one

\footnotetext{
6 http://mastweb.stsci.edu/ps1casjobs/

7 https://irsa.ipac.caltech.edu/docs/program interface/ztf_lightcurve_api.html

8 For example wget https://irsa.ipac.caltech.edu/cgi-bin/ ZTF/nph_light_curves?POS=CIRCLE+352.573853+53.883614+ 0.00028\&NOBS_MIN=3\&BAD_CATFLAGS_MASK $=32768 \& F O R M A T=$ ipac_table-0352.573853.tbl to select data within $0.00028^{\circ}\left(1^{\prime \prime}\right)$ around $(\mathrm{RA}, \mathrm{Dec})=(352.573853,53.883614)$ filtering out bad data and with at least three observations.

9 http://surveys.roe.ac.uk/vsa/index.html

${ }^{10}$ http://horus.roe.ac.uk: 8080/vdfs/VcrossID_form.jsp? disp=adv

11 After preparing a file with RA and Dec, choosing a pairing radius of $3^{\prime \prime}$, and selecting 'all nearby sources' as option the query is:
}

SELECT \#upload.*, \#proxtab.distance, d.RA, d.Dec, d.filterID, d.mjd, d.aperMag3, d.aperMag3err, d.ppErrBits FROM \#upload left

outer join \#proxtab on \#upload.upload_id=upid left outer join vvvDetection as $d$ on d.objID=archiveID left outer join multiframe on multiframe.multiframeId=d.multiframeID where frametype like 'tilestack' order by upload_id

12 J/ApJS/190/203/var. 
Table 2. Fit results of the C-star sample (first entries).

\begin{tabular}{|c|c|c|c|c|c|c|c|c|c|c|c|c|}
\hline $\begin{array}{l}\text { RA } \\
\text { (deg) }\end{array}$ & $\begin{array}{r}\text { Dec } \\
(\mathrm{deg}) \\
\end{array}$ & $\begin{array}{r}\text { Period } \\
\text { (days) }\end{array}$ & $\begin{array}{r}d \\
(\mathrm{kpc}) \\
\end{array}$ & $\begin{array}{r}A_{V} \\
(\mathrm{mag}) \\
\end{array}$ & $\begin{array}{l}T_{\text {eff }} \\
(\mathrm{K})\end{array}$ & $\begin{array}{r}L \\
\left(L_{\odot}\right) \\
\end{array}$ & $\tau_{0.5}$ & $\begin{array}{r}T_{\mathrm{c}} \\
(\mathrm{K})\end{array}$ & $f$ & $p$ & $f$ & $\begin{array}{r}\dot{M} \\
\left(M_{\odot} \mathrm{yr}^{-1}\right)\end{array}$ \\
\hline 19.463942 & 67.231445 & 1047 & 2.74 & 3.73 & 3300 & $13214 \pm 75$ & $170 \pm 1.5$ & $1000 \pm 0$ & 0 & $2.0 \pm 0.0$ & 0 & $0.262 \mathrm{E}-04$ \\
\hline 349.802521 & 17.192619 & 746 & 0.77 & 0.23 & 2700 & $6661 \pm 108$ & $95 \pm 0.9$ & $1000 \pm 0$ & 0 & $2.0 \pm 0.0$ & 0 & $0.893 \mathrm{E}-05$ \\
\hline 353.599762 & 43.504013 & 629 & 0.67 & 0.36 & 2500 & $4729 \pm 66$ & $15 \pm 0.2$ & $716 \pm 7$ & 1 & $2.0 \pm 0.0$ & 0 & $0.206 \mathrm{E}-05$ \\
\hline 124.826309 & -21.737400 & 939 & 2.80 & 0.20 & 3300 & $10621 \pm 219$ & $136 \pm 3.9$ & $1000 \pm 0$ & 0 & $2.0 \pm 0.0$ & 0 & $0.167 \mathrm{E}-04$ \\
\hline 287.486908 & 9.447611 & 1071 & 2.32 & 5.45 & 2800 & $13014 \pm 341$ & $99 \pm 1.6$ & $1201 \pm 0$ & 0 & $2.0 \pm 0.0$ & 0 & $0.977 \mathrm{E}-05$ \\
\hline 237.773834 & -56.890007 & 951 & 2.51 & 1.54 & 2500 & $10605 \pm 262$ & $57 \pm 0.5$ & $655 \pm 17$ & 1 & $2.0 \pm 0.0$ & 0 & $0.149 \mathrm{E}-04$ \\
\hline 323.345001 & 56.743065 & 930 & 2.03 & 2.54 & 2600 & $10334 \pm 187$ & $109 \pm 2.6$ & $1000 \pm 0$ & 0 & $2.0 \pm 0.0$ & 0 & $0.122 \mathrm{E}-04$ \\
\hline 75.631233 & -68.093285 & 1884 & 50.00 & 0.22 & 2700 & $7897 \pm 44$ & $251 \pm 1.8$ & $1000 \pm 0$ & 0 & $2.0 \pm 0.0$ & 0 & $0.304 \mathrm{E}-04$ \\
\hline 76.023376 & -68.394501 & - & 50.00 & 0.22 & 3100 & $5992 \pm 10$ & $248 \pm 0.9$ & $1000 \pm 0$ & 0 & $2.0 \pm 0.0$ & 0 & $0.262 \mathrm{E}-04$ \\
\hline 79.548790 & -70.507469 & - & 50.00 & 0.22 & 3200 & $9496 \pm 18$ & $273 \pm 1.0$ & $1000 \pm 0$ & 0 & & 0 & $0.330 \mathrm{E}-04$ \\
\hline 79.701599 & -69.55956 & - & 50.00 & 0.22 & 310 & $6935 \pm 10$ & $189 \pm 0.6$ & $1000 \pm 0$ & 0 & & 0 & $0.199 \mathrm{E}-04$ \\
\hline 81.419411 & -70.14087 & - & 50.00 & 0.2 & 30 & $4000 \pm 10$ & $205 \pm 1.1$ & $1000 \pm 0$ & 0 & $2.0 \pm 0.0$ & 0 & $0.167 \mathrm{E}-04$ \\
\hline 82.407959 & -72.831322 & 678 & 50.00 & 0.2 & 280 & $5498 \pm 18$ & $125 \pm 0.7$ & $1000 \pm 0$ & 0 & $2.0 \pm 0.0$ & 0 & E-04 \\
\hline 87.60 & -69.934212 & 1110 & 50.00 & 0.2 & 2600 & $10351 \pm 12$ & $111 \pm 3.8$ & $743 \pm 15$ & 1 & $2.0 \pm 0.0$ & & $0.245 \mathrm{E}-04$ \\
\hline 78.257469 & -69.564110 & - & 50.00 & 0.2 & 2600 & $6381 \pm 21$ & $274 \pm 1.4$ & $1000 \pm 0$ & 0 & $2.0 \pm 0.0$ & 0 & $0.307 \mathrm{E}-04$ \\
\hline 82.684006 & -71.716766 & 954 & 50.00 & 0.22 & 3600 & $8233 \pm 29$ & $46 \pm 1.8$ & $269 \pm 5$ & 1 & $2.0 \pm 0.0$ & 0 & $0.103 \mathrm{E}-03$ \\
\hline 87.249886 & -70.556229 & 3434 & 50.00 & 0.22 & 3000 & $12280 \pm 28$ & $85 \pm 1.4$ & $283 \pm 3$ & 1 & $2.0 \pm 0.0$ & 0 & $0.182 \mathrm{E}-03$ \\
\hline 89.161446 & -67.892776 & 1220 & 50.00 & 0.22 & 4000 & $20161 \pm 136$ & $74 \pm 0.8$ & $1000 \pm 0$ & 0 & $2.0 \pm 0.0$ & 0 & $0.121 \mathrm{E}-04$ \\
\hline 5.961981 & 62.636379 & 1065 & 4.98 & 2.21 & 2800 & $13619 \pm 261$ & $93 \pm 2.3$ & $1000 \pm 0$ & 0 & $2.0 \pm 0.0$ & 0 & $0.116 \mathrm{E}-04$ \\
\hline 38.251453 & 58.035065 & 827 & 2.33 & 1.69 & 2400 & $8209 \pm 803$ & $46 \pm 1.5$ & $642 \pm 33$ & 1 & $2.0 \pm 0.0$ & 0 & $0.119 \mathrm{E}-04$ \\
\hline 39.529259 & 54.587803 & 905 & 4.63 & 1.04 & 2400 & $9856 \pm 223$ & $75 \pm 2.7$ & $1000 \pm 0$ & 0 & $2.0 \pm 0.0$ & 0 & $0.770 \mathrm{E}-05$ \\
\hline 41.103512 & 55.187542 & 477 & 3.95 & 1.64 & 2400 & $2691 \pm 78$ & $78 \pm 5.6$ & $514 \pm 32$ & 1 & $2.0 \pm 0.0$ & 0 & $0.187 \mathrm{E}-04$ \\
\hline 47.976738 & 60.956123 & 1163 & 8.11 & 3.52 & 3300 & $16391 \pm 266$ & $68 \pm 1.9$ & $1000 \pm 0$ & 0 & $2.0 \pm 0.0$ & 0 & $0.899 \mathrm{E}-05$ \\
\hline 57.080860 & 44.701607 & & & & & & & $842 \pm 19$ & 1 & & 0 & $0.266 \mathrm{E}-05$ \\
\hline 72.919174 & & & & & & & & & 0 & & 0 & $0.729 \mathrm{E}-05$ \\
\hline 74.69 & & & & & & & $40 \pm 1.5$ & $736 \pm$ & 1 & $2.0 \pm 0.0$ & 0 & $859 \mathrm{E}-05$ \\
\hline 76.270149 & -68.963379 & 938 & 50.00 & 0.22 & 3000 & $9645 \pm 154$ & $83 \pm 2.0$ & $1000 \pm 0$ & 0 & $2.0 \pm 0.0$ & 0 & $0.850 \mathrm{E}-05$ \\
\hline 76.646332 & -70.280640 & 554 & 50.00 & 0.22 & 5000 & $8943 \pm 92$ & $22 \pm 0.8$ & $682 \pm 15$ & 1 & $2.0 \pm 0.0$ & 0 & $0.592 \mathrm{E}-05$ \\
\hline 78.003212 & -70.540047 & 1182 & 50.00 & 0.22 & 4000 & $15047 \pm 77$ & $55 \pm 0.5$ & $1000 \pm 0$ & 0 & $2.0 \pm 0.0$ & 0 & $0.740 \mathrm{E}-05$ \\
\hline 82.525955 & -70.511375 & 807 & 50.00 & 0.22 & 3200 & $9845 \pm 115$ & $64 \pm 1.1$ & $1200 \pm 0$ & 0 & $2.0 \pm 0.0$ & 0 & $0.474 \mathrm{E}-05$ \\
\hline 85.336433 & -69.078796 & 895 & 50.00 & 0.22 & 2700 & $9363 \pm 84$ & $63 \pm 2.7$ & $764 \pm 23$ & 1 & $2.0 \pm 0.0$ & 0 & $0.108 \mathrm{E}-04$ \\
\hline 87.485626 & -70.886604 & 1041 & 50.00 & 0.22 & 2600 & $17597 \pm 264$ & $27 \pm 2.1$ & $794 \pm 26$ & 1 & $2.0 \pm 0.0$ & 0 & $0.543 \mathrm{E}-05$ \\
\hline 91.000160 & 7.431088 & 696 & 1.28 & 0.83 & 2400 & $5818 \pm 103$ & $31 \pm 0.5$ & $775 \pm 19$ & 1 & $2.0 \pm 0.0$ & 0 & $0.353 \mathrm{E}-05$ \\
\hline 91.039764 & 47.795067 & 934 & 5.15 & 0.43 & 2400 & $10507 \pm 225$ & $86 \pm 3.0$ & $1000 \pm 0$ & 0 & $2.0 \pm 0.0$ & 0 & $0.935 \mathrm{E}-05$ \\
\hline 95.182762 & -4.558214 & 1795 & 23.15 & 0.77 & 2700 & $39535 \pm 1639$ & $257 \pm 11.1$ & $1000 \pm 0$ & 0 & $2.0 \pm 0.0$ & 0 & $0.700 \mathrm{E}-04$ \\
\hline 99.256760 & -1.450483 & 854 & 4.36 & 2.71 & 2400 & $8762 \pm 243$ & $42 \pm 2.3$ & $1000 \pm 0$ & 0 & $2.0 \pm 0.0$ & 0 & $0.371 \mathrm{E}-05$ \\
\hline
\end{tabular}

Notes. Columns 1 and 2 give the RA and Dec in decimal degrees, Col. 3 gives the pulsation period in days, Col. 4 gives the distance in kpc, Col. 5 gives the IS reddening $A_{V}$, Col. 6 gives the effective temperature in Kelvin, Col. 7 gives the luminosity and error in $L_{\odot}$, Col. 8 gives the optical depth at $0.5 \mu \mathrm{m}$, Col. 9 gives the condensation temperature with error in Kelvin, Col. 10 indicates if the condensation temperature was fitted (1), or fixed (0). In the latter case the error is set to $0 \mathrm{~K}$, Col. 11 gives the slope of the density law with error, Col. 12 indicates if the slope was fitted (1), or fixed (0). In the latter case the error is set to 0, Col. 13 gives the gas MLR in $M_{\odot} \mathrm{yr}^{-1}$. The first entries are the template ERO sources from Table 1 . The remainder are listed in order of RA. The full table is available at the CDS.

source), from the Catalina Sky Survey (CSS; Drake et al. 2009; three sources), the Bochum Galactic Disk Survey (GDS; Hackstein et al. 2015; two sources), and $K$-band photometry from Kerschbaum et al. (2006) with photometry from the literature being added (two sources). In addition, VMC $K$-band data from Groenewegen et al. (2020) were refitted with an improved initial period from the present work.

\subsection{Period analysis and LC modelling}

The automatic analysis of the LCs was carried out with the Fortran codes available in numerical recipes (Press et al. 1992) as described in Appendix A of Groenewegen (2004) and modified to analyse the VMC $K$-band data as described in Groenewegen et al. (2020). The Fourier analysis was done using the subroutine FASPER. However, as a cross-check, most of the stars in the sample were analysed manually with the code
PERIOD04 (Lenz \& Breger 2005) as well. After an initial guess for the period was determined (either through the automatic routine, a period found in the literature, or from the manual fitting of the LC), a function of the form

$m(t)=m_{0}+A \sin \left(2 \pi t e^{f}\right)+B \cos \left(2 \pi t e^{f}\right)$,

was fitted to the LC using the weighted linear least-squares fitting routine MRQMIN. This results in the parameters listed in Tables A.2, A.4, and A.6, namely mean magnitudes $\left(m_{0}\right)$, periods $(\exp (-f))$, and amplitudes $\left(\sqrt{A^{2}+B^{2}}\right)$ with their associated uncertainties. Equation (1) implies that the LC can be described by a single period. It is well known that the LCs of LPVs are not strictly single-periodic (as many of the fitted LCs show). However with the limited number of data points available one is in general not able to comment on the presence of more than one period.

A comparison of the LC with the fit sometimes suggested that alternative periods may be possible as well. These 
cases were inspected by the manual fitting of the LC using PERIOD04, and alternative periods (denoted Palt) are sometimes indicated in the comments for Tables A.2, A.4, and A.6. The tables also include the reduced $\chi^{2}$, defined as $\chi_{\mathrm{r}}^{2}=$ $\sum_{i}\left(\left(\left(m_{i}-o_{i}\right) / \sigma_{o_{i}}\right)^{2}\right) /\left(N-N_{\mathrm{p}}\right)$, with $m, o$ and $\sigma_{o}$ indicating the model magnitude, the observed magnitude, and the error, respectively, $N$ is the number of data points, and $N_{\mathrm{p}}=1$ or 4 , depending on whether Eq. (1) is fitted without or with the period.

The Fourier analysis and the LC modelling were done on the $W 1$ and $W 2$ data separately. If the total time span of the data is less than 400 days, or the total number of data points is less than eight, the LC fitting process was terminated. This resulted in cases where only one LC was generated and these cases were inspected more closely. In most cases $(\sim 100$ stars $)$ the data refer to a fake source in the AllWISE catalogue (see Sect. 6.1) and that source was removed from the fitting all together; in $\sim 15$ cases, the fit seemed reliable and in those cases the fitting process was carried out in the other filter using the available data, even if there were fewer than eight data points covering a shorter time span.

\section{Literature data}

To characterise the sources better, the target list was correlated with other databases. From the SIMBAD database, some common names and the object type were retrieved using a search radius of $3^{\prime \prime 13}$.

Real sources that were detected in the $W 1$ and $W 2$ filters are expected to have been detected in other IR bands as well. To verify that, the target list was correlated with the following photometric catalogues which are all available through VizieR: 2MASS (Cutri et al. 2003) using a search radius of 1.0"; the Akari/IRC MIR all-sky survey (Ishihara et al. 2010) using a search radius of 3.0"; the Midcourse Space Experiment (MSX) (Egan et al. 2003) using a search radius of 5.0"; the Herschel infrared Galactic Plane Survey (Hi-GAL) $70 \mu \mathrm{m}$ catalogue (Molinari et al. 2016), using a search radius of 4.5"; the Galactic Legacy Infrared Midplane Survey Extraordinaire (GLIMPSE) (Benjamin et al. 2003; Spitzer Science Center 2009) using a search radius of $3.0^{\prime \prime}$, and the MIPSGAL survey at $24 \mu \mathrm{m}$ (Gutermuth \& Heyer 2015) using a search radius of 2.0". The first three surveys are all-sky, while the latter three are surveys mainly of the galactic plane.

Figure 2 shows that many sources are located in the galactic plane which has been surveyed extensively for $\mathrm{OH}$ maser emission. A double-peaked $\mathrm{OH}$ profile is a characteristic of evolved $\mathrm{O}$-stars. The target list was correlated with the $\mathrm{OH}$ database of Engels \& Bunzel (2015) using a search radius of 3.5". OH maser sources in the MCs (Goldman et al. 2017, 2018) were also considered. The target list is also correlated with the classification of over 11000 sources from IRAS LRS spectra (Kwok et al. 1997) that contains information on the dust species and continuum shape in the $8-23 \mu \mathrm{m}$ region using a search radius of $12^{\prime \prime}$, and with the compilation of spectral types (Skiff 2014) using a search radius of $3.2^{\prime \prime}$.

Finally, the target list was correlated with a number of catalogues containing extra galactic sources. There are no matches with the catalogues of 'Quasars and Active Galactic Nuclei' (13th ed., Véron-Cetty \& Véron 2010), the 'Large Quasar Astrometric Catalogue 4' (Gattano et al. 2018), and

\footnotetext{
13 With an exception for one well-known source which was located at 4. $1^{\prime \prime}$ from the AllWISE coordinates. The SIMBAD query was done in June 2020.
}

the 'SDSS quasar catalogue' (DR16, Lyke et al. 2020). There are two matches in 'The Million Quasars' catalogue (version 7.2, April 2021; Flesch 2015), that lists a source at $\mathrm{RA}=286.187653$, Dec $=+48.885826$ as having a $91 \%$ probability of being a QSO with a redshift of 0.700 , and at $\mathrm{RA}=80.513848$, Dec $=-68.322622$ as having a $69 \%$ probability of being a QSO with a redshift of 0.900 .

There are more matches in catalogues listing AGN and QSO candidates, such as the 'Gaia DR2 quasar and galaxy classification' (Bailer-Jones et al. 2019, 60 matches), the ,QSO candidates catalogue with APOP and ALLWISE, (Guo et al. 2018, 3 matches), and the 'The WISE AGN candidates catalogues' (the R90 90\% reliability catalogue, Assef et al. 2018, 2 matches). The recent Gaia EDR3 list of AGN and QSOs (Gaia Collaboration 2021a,b) contains 3 matches within 1 arcsec. These matches are listed in Tables A.1, A.3, and A.5 from which it is clear that many are actually stellar sources. For example, of the 60 candidate QSOs in Bailer-Jones et al. (2019) 39 show LPV-like pulsations (see Sect. 6.4). The target list was also correlated with the catalogue of Solarz et al. (2017). They used a novel approach (one-class support vector machines, OCSVM) to identify anomalous patterns in AllWISE colours. Their method allowed them to detect anomalies (e.g. objects with spurious photometry), and also real sources such as a sample of heavily reddened AGN/quasar candidates.

\section{Results}

The results of the literature search and the period analysis are compiled in Tables A.1-A.6. There is a table listing the results of the literature search and a table listing the results of the period analysis for three classes of objects: 1224 bona fide stellar sources with a period analysis based on WISE data (Tables A.1 and A.2); 118 bona fide stellar sources without sufficient WISE data for the LC analysis in both filters, but possibly with a period from the literature or analysis of literature data (Tables A.3 and A.4); and 650 other sources that may contain a few extra galactic objects, but most are not bona fide sources (Tables A.5 and A.6).

The distinction between the bona fide stellar sources and those that (very likely) are not is based on the number of associations with a SIMBAD object and the other external catalogues mentioned in Sect. 4, an inspection of the LC, and the result of the LC fitting. Signatures of a fake source are no, or only one association with an external catalogue (often close to the limit of the search radius used), and a LC with a few points. They are further discussed in Sect. 6.1.

Tables A.1, A.3, and A.5 contain the results of the literature search and they include the distance to the closest SIMBAD object and the other photometric catalogues discussed in Sect. 4. This also includes the blue and red velocity of any $\mathrm{OH}$ maser emission, the IRAS LRS classification, and the spectral type. Tables A.2, A.4, and A.6 contain the periods quoted in the literature, the periods derived from fitting literature data, and the results of fitting the WISE data (period with error, amplitude with error, mean magnitude with error, and the reduced $\chi^{2}$ in the $W 1$ and $W 2$ filters). Examples of the lightcurve and the fits are shown for both the WISE data (Fig. B.1) and the other fitted data from the literature (Figs. B.2-B.6). The complete set of fitted LCs is available online ${ }^{14}$. Figures B.7-B.11 show the LCs for the datasets with a limited number of matches that can fit in a single figure.

${ }^{14}$ https://doi.org/10.5281/zenodo. 5825878 


\section{Discussion}

\subsection{AIIWISE sources that are very likely not real}

In inspecting the coordinates of the mostly fake objects (Table A.5) it is striking to observe that they can be very similar, and in fact many are related to very bright objects (hereafter, 'clones'). The most conspicuous example are the clones of CW Leo. CW Leo is located at $(\mathrm{RA}, \mathrm{Dec})=(146.989193$, +13.278768 ) and it is not present in the AllWISE catalogue. In the target list, there are 42 sources located up to $17^{\prime}$ from this position not associated with any other known source. Other well-known IR bright sources have clones, including AFGL $3068(\mathrm{RA}, \mathrm{Dec}=349.802533,+17.192628, W 1=$ $4.7 \mathrm{mag}, W 2$ is unreliable; 16 sources up to $9^{\prime}$ ), VY CMa (RA, Dec $=110.7430362,-25.7675659, W 1 \sim 1.7 \mathrm{mag}$ and $W 2 \sim$ $3.4 \mathrm{mag}$ but both are unreliable; nine sources up to $10^{\prime}$ ), or IRC +10420 (RA, Dec $=291.700408,+11.354634$, not in AllWISE; seven sources up to $\left.9^{\prime}\right)$. For a slightly fainter source such as IRAS 08171-2134 (RA, Dec $=124.8263077,-21.737400$, $W 1=7.3 \mathrm{mag}$ and $W 2=3.8 \mathrm{mag})$, the number is reduced to three sources up to $1.2^{\prime}$ distance.

Some of these clones have LCs that are periodic (see Fig. 3) with periods in agreement with the literature values. These are CW Leo $P=643 \pm 1.4$ days (the weighted mean of the periods obtained in the $W 1$ and $W 2$ bands, cf. Table A.6) compared to $P=639 \pm 4$ (e.g. Groenewegen et al. 2012 and references therein), IRAS 15194-5115 $P=565 \pm 2.3$ days compared to $P=580$ (Le Bertre 1992) and $P=576$ (Whitelock et al. 2006), IRC $+20326 P=549 \pm 3.6$ days compared to $P=540$ (Uttenthaler et al. 2019), AFGL $3116 P=630 \pm 3.3$ days compared to $P=620$ (Jones et al. 1990) or $P=599$ (Drake et al. 2014), and AFGL $2135 P=619 \pm 13$ days compared to $P=655$ (Whitelock et al. 2006).

The number of fake sources is almost one-third of the sample and one may wonder if these could have been filtered out using flags available in the AllWISE catalogue. Both Chen et al. (2018) and Uchiyama \& Ichikawa (2019) used additional selection criteria, for example on the photometric quality ( $\left.p h \_q u a l\right)$, contamination and confusion flag (cc_flags), variability flag (var_flag), fraction of saturated pixels (w? sat), or poor PSF profile fitting ( $w$ ? rchi2), where '?' stands for ' 1 ' or ' 2 ' depending on the filter.

Among the fake sources $86 \%$ have a $c c \_f l a g$ in the $W 1$ and $W 2$ filter which is not equal to '00', but so do $77 \%$ of the bona fide sources. The fake sources also do not necessarily have poor photometric quality flags $(77 \%$ in fact have a ph_flag in the $W 1$ and $W 2$ filter of 'AA'). Other flags were inspected, but in conclusion, many bona fide sources with good quality data would be eliminated by applying stricter selection criteria, although this implies including a significant number of fake sources.

\subsection{Sources in the VVV survey}

Of the 122 sources analysed by Ferreira Lopes et al. (2020), 51 are listed with a period of 1 day (when rounded to one digit) and another ten with periods below 10 days. Out of the remaining 61 , only nine periods agree to within $10 \%$ with the periods derived here; while in 32 cases, the difference in period is more than a factor of two, and up to a factor of ten. The reason for this large discrepancy is very likely related to the frequency range that was explored in Ferreira Lopes et al. (2020), which is namely larger than $\frac{2}{T} d^{-1}$, with $T$ being the time span (see Sect. 4.2 of that paper). This time span is not explicitly given and likely varies from source to source, but it probably leads to
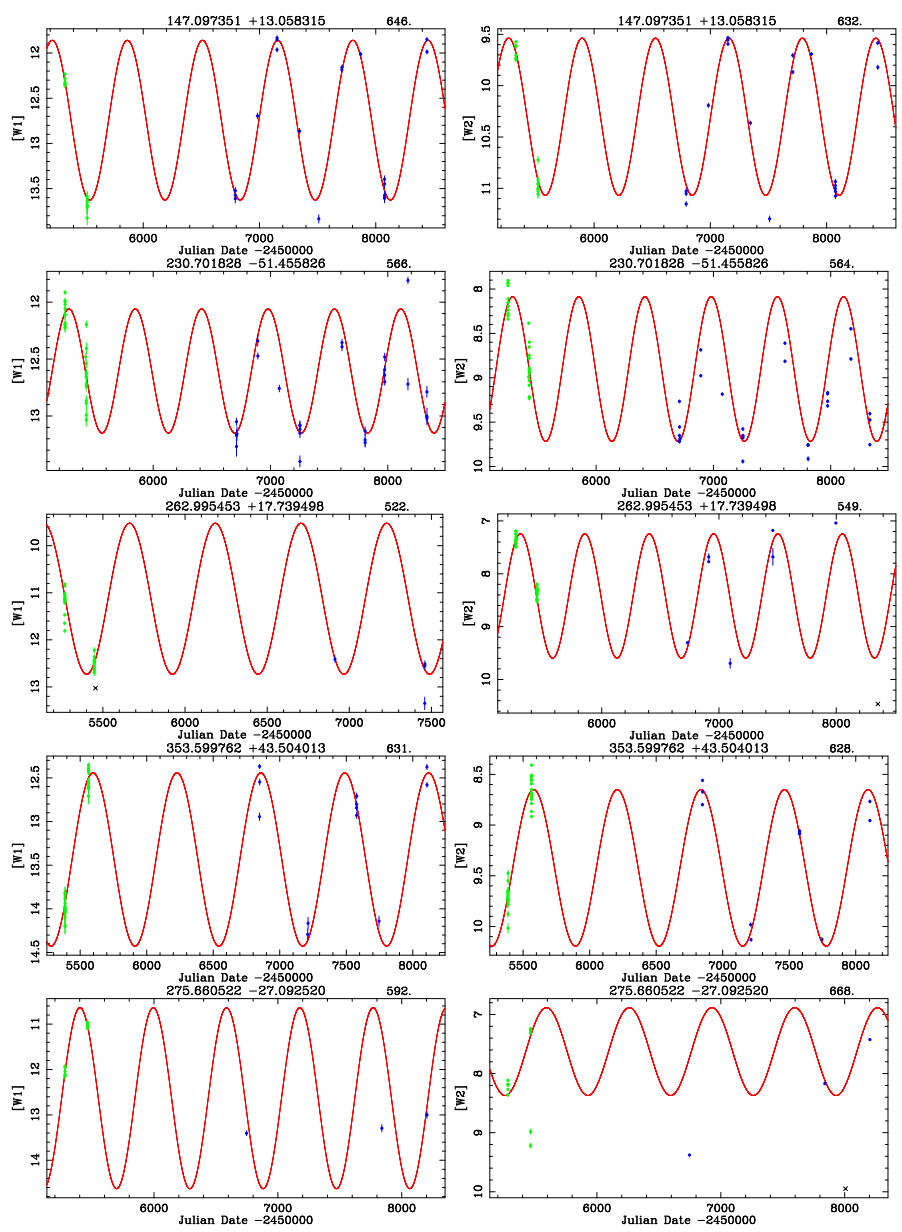

Fig. 3. Examples of LC fits ( $W 1$ in the left-hand panel and $W 2$ in the right-hand panel) to clones of CW Leo (RA = 147.097351), IRAS 15194-5115 $(\mathrm{RA}=230.701828), \quad \mathrm{IRC}+20326(\mathrm{RA}=262.995453)$, AFGL 3116 (RA = 353.599762), and AFGL 2135 (RA = 275.660522). The identifier is listed on top of each panel, with the period to the right.

a lower frequency limit that is too large. In fact, an earlier paper by the VVV team (Contreras Peña et al. 2017) that searched for high-amplitude infrared variable stars lists different periods for a number of stars (they do not state what frequency range they searched). Out of the ten sources in the sample with periods in Contreras Peña et al. (2017), nine have a period from WISE data of which seven agree to within $10 \%$ and all nine to within $20 \%$. The periods listed in Ferreira Lopes et al. (2020) for those stars are all incorrect (three have a period of one day or periods are too small by factors of 1.7 to 3.2 ).

As an additional check, for a sample of 49 stars the $K$-band data from the VVV sources were reanalysed using the publically available data. For 42, the periods derived in this way agreed to within $10 \%$ with the period from the WISE data. All the revised periods and LCs are available in the Appendices, as explained at the end of Sect. 5.

\subsection{Non-variable OHIR stars}

In a recent paper, Kamizuka et al. (2020) investigated the NIR brightening of non-variable $\mathrm{OH} / \mathrm{IR}$ stars. The $\mathrm{OH}$ maser emission of $\mathrm{OH} / \mathrm{IR}$ stars on the AGB is expected to follow the pulsation period of the underlying star, see Sect. 2.2. However, non-variable $\mathrm{OH} / \mathrm{IR}$ stars are known to exist (Herman \& Habing 1985) and this is expected to happen in the transition from 
the AGB to the P-AGB phase when large-amplitude pulsations stop.

Kamizuka et al. (2020) selected 16 stars from the sample in Herman \& Habing (1985), which had the smallest variability amplitudes in their OH/IR maser emission. They established NIR multi-epoch data for six objects, based on archival data from 2MASS (Cutri et al. 2003), UKIDSS (Lucas et al. 2008), and data taken with the Okayama Astrophysical Observatory Wide Field Camera (OAOWFC; Yanagisawa et al. 2019). For all six stars, they derived a brightening in the $K$-band in the range

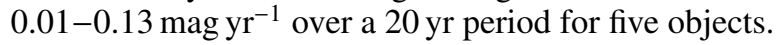

Of the 16 objects studied in Kamizuka et al. (2020), four are in the WISE sample: $\mathrm{OH} 17.7-2.0(\mathrm{RA}=277.627928)$ and $\mathrm{OH} 31.0+0.0(\mathrm{RA}=281.921407)$ that are not among the six for which Kamizuka et al. (2020) determined a NIR brightening, and $\mathrm{OH} 31.0-0.2(\mathrm{RA}=282.179217)$ and $\mathrm{OH} 37.1-0.8$ $(\mathrm{RA}=285.526137)$ for which they determined a brightening of $2.04 \mathrm{mag}$ over 2250 days $\left(0.33 \mathrm{mag} \mathrm{yr}^{-1}\right)$ and $0.35 \mathrm{mag}$ over 2170 days $\left(0.06 \mathrm{mag} \mathrm{yr}^{-1}\right)$, respectively, however based on only two data points in both cases.

The $W 1$ and $W 2$ LCs for these four objects are shown in the top four panels of Fig. 4. We note that OH 17.7-2.0 is becoming fainter by $\sim 0.7(W 1)$ and $\sim 0.4$ (W2) mag over $\sim 3200$ days

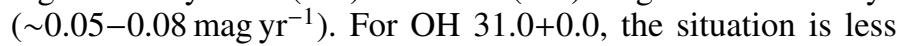
clear in $W 1$, but in the $W 2$ filter there is a faintening by $\sim 0.4$ mag. Also, $\mathrm{OH} 31.0-0.2$ is not clearly brightening or faintening. The formal LC fitting gives very long periods, which must be taken with caution as the time span of the WISE observations covers less than half of the putative pulsation period. The two $K$-band data points considered by Kamizuka et al. (2020) were taken at epochs 2453536 and 2455787. The WISE LCs do show a brightening between 2455200 and the last $K$-band epoch. Furthermore, $\mathrm{OH} 37.1-0.8$ shows a marginal brightening of order $0.2 \mathrm{mag}$ over $8.7 \mathrm{yr}$ in both filters.

Along similar lines, Lewis (2002) observed the $\mathrm{OH}$ maser emission of 328 stars after $12 \mathrm{yr}$ again to find four with undetectable emission at re-observation, and one in 'terminal decline'. One of these five stars is in the sample (IRAS 18455+0448, RA = 282.009613) and its WISE LC is shown in the bottom panels of Fig. 4. Its WISE emission is consistent with no variation.

To investigate this more systematically, the light curves of all stars in the sample were inspected that either have a SIMBAD classification as an OH/IR star (Col. 5 in Tables A.1, A.3, and A.5) or an entry in the database of Engels \& Bunzel (2015) (Col. 12/13 in those tables). All stars with a period from analysis of the WISE data, a period from the literature or from refitting literature data, as well as stars not detected in $\mathrm{OH}$ and classified different from an O-rich star, were removed. Twenty new candidate non-variable $\mathrm{OH} / \mathrm{IR}$ stars were identified. They are labelled with 'nvoh' in Col. 20 in Tables A.2 and A.4. The five objects in the sample previously identified in the literature are labelled with 'NVOH' in those tables.

\subsection{Selecting LPVs}

The selection of (candidate) LPVs from the WISE data is based on the amplitude. The geometric mean of the amplitude in the $W 1$ and $W 2$ filters $(\mathrm{AmpW})$ and the errors therein $\left(\sigma_{\mathrm{AmpW}}\right)$ were calculated. LPV candidates are those with AmpW $>0.2$ mag, AmpW $/ \sigma_{\mathrm{AmpW}}>2.5$ and a $S / N>6$ in the amplitude detection in either the $W 1$ or $W 2$ filter. One well-known LPV (AFGL 3068, $\mathrm{RA}=349.802533$ ) was added manually to this list. The cut in amplitude is chosen to correspond roughly with typical cut-off
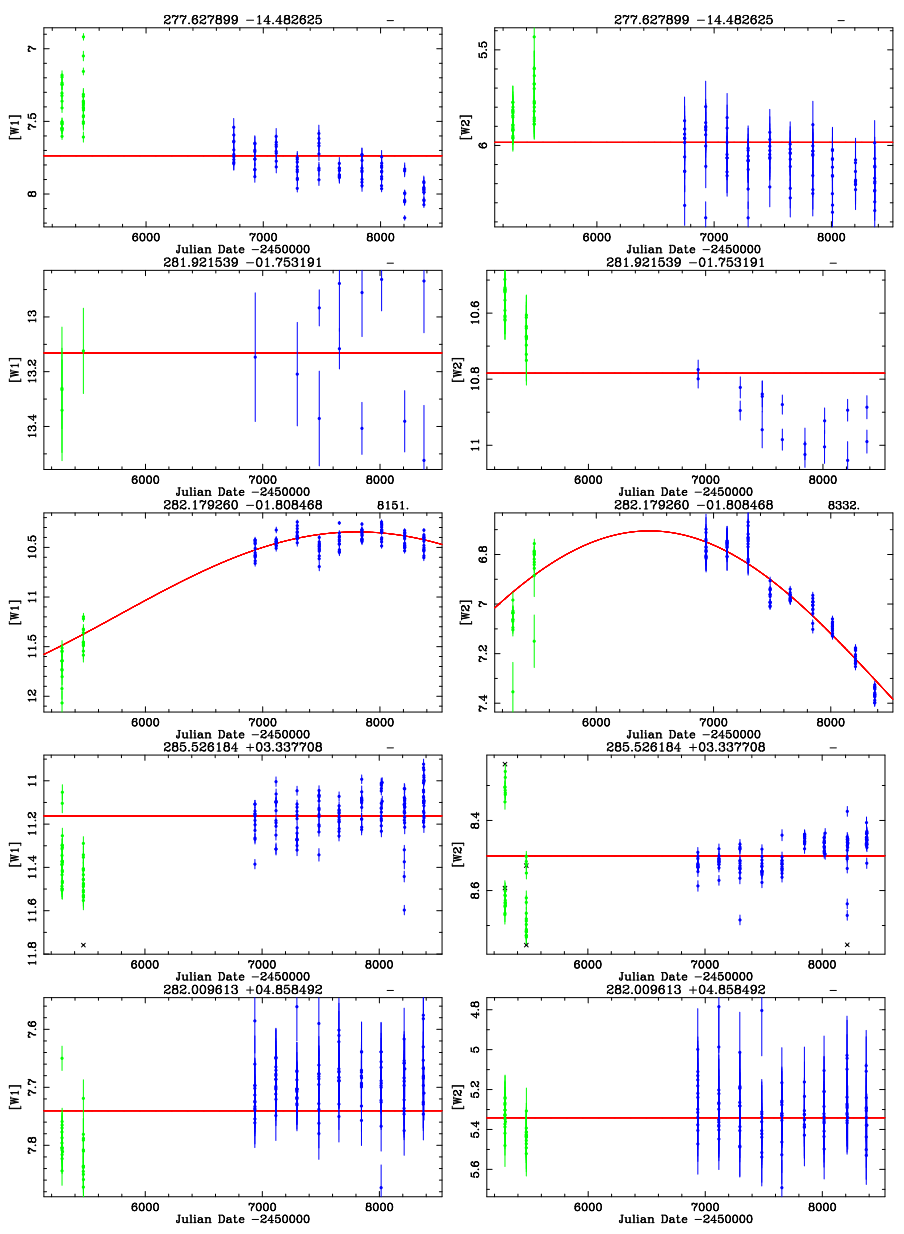

Fig. 4. WISE LCs of non-variable OH/IR stars in the sample.

values of about 0.45 mag used in the $I$-band and of about 0.2 mag in the and $K$-band. The cuts on the $\mathrm{SN}$ ratio were determined empirically by visually inspecting the LCs and fits to the LCs of stars selected in this way, and those that are not. In this way 752, LPVs were selected, of which 356 appear to be newly classified as such. They are marked 'LPV' in Tables A.1 and A.3. However, there always remain some borderline cases where the LC of an LPV candidate appears noisy, and only higher precision photometry over a sufficiently long time span may resolve the variable nature of some sources. One hundred fourty-five sources have periods longer than 1000 days $^{15}$ of which 109 are new (13 had previously quoted periods below 1000 , typically 160-700 days), which is a significant increase in the 16 template sources in Table 1 from Menzies et al. (2019). The referee directed us to the paper by Chen et al. (2020) which analysed and classified a large number of variable stars based on ZTF data. A comparison with the periods derived in the present paper based on WISE data and ZTF data is presented in Appendix E.

The list of non-LPV candidates selected in this way (the complementary sample) contains interesting sources, some of which are periodic, but with a smaller amplitude, or where a good LC fit is obtained in one filter only, or they show peculiar LCs. These sources are marked 'PER' in Tables A.2 and A.4. They include known OH/IR stars, also sometimes with a period derived from the literature, but with poor WISE data in one filter, in addition to Sakurai's object (see Evans et al. 2020 for a

${ }^{15}$ Sources $(81.850469,-69.662488)$ and $(83.154874,-67.115672)$ have periods close to this limit of 993 and 988 days, respectively. 
detailed discussion on its $K$ and WISE LC). Examples of LCs for such sources are displayed in Fig. B.12.

\subsection{EROs and the mass return to the ISM}

As the (rare) C-rich stars with the highest MLRs dominate the mass return by AGB stars to the ISM (see references in the introduction) it is of interest to identify new objects in this class, both in the Galaxy and the MCs. The template sample in Table 1 of EROs is based on the shape of the spectrum in the MIR (a red flat continuum or the $\mathrm{SiC}$ feature in absorption), but spectral data are generally not available, only photometric data are. Based on the colours in Table 1, the spectral energy distributions (SEDs) of the 316 objects with $W 2-W 3>3.0$ mag were constructed using data in the literature. For a subset of 141 stars, MIR spectra were available. The SEDs were fitted with the dust radiative transfer code more of DUSTY (MoD, Groenewegen 2012), which is an extension of the radiative transfer code DUSTY (Ivezić et al. 1999), allowing the derivation of luminosities and MLRs; the details are given in Appendix C.

Distances to the galactic sources were derived as follows. Based on the C-rich objects in the MCs the following periodluminosity (PL) relation was derived (see details in Appendix C and shown in Fig. C.1),

$M_{\mathrm{bol}}=-5.07 \cdot(\log P-2.8)-4.47 \mathrm{mag}$,

based on 31 objects and with an rms of 0.31 mag. This PL relation was then applied to the Galactic objects for which a period was available, $\mathrm{O}$ - and $\mathrm{C}$-rich alike. The PL relation was derived using stars with periods up to $\log P \approx 3.1$ (about 1260 days), while the longest period for which it has been applied has a period of about 2600 days $(\log P \approx 3.4$ ). For $\mathrm{C}$-stars without a period the median luminosity of $7100 L_{\odot}$ of the MCs objects with a period was used; for the O-rich objects without a period an arbitrary distance of $2.0 \mathrm{kpc}$ was adopted. Interstellar (IS) reddening was included (see Appendix C). That notion that the PL relation derived for ERO C-stars in the MCs would hold for Galactic C-stars, and for O-stars, is an assumption made here. Data for less reddened and shorter period ( $P \lesssim 400$ days) Miras are consistent with the premise that any differences are small (Whitelock et al. 2008). We note that, to first order, ignoring the dependence of the reddening on distance, $L \sim d^{2}$ and $\dot{M} \sim d$ if the reader prefers another distance.

Based on the MIR spectra and the fitting of the SED, the sample was divided into $197 \mathrm{C}$-rich and 119 O-rich sources. Of the C-rich sources, 18 belong to the sample of Galactic and LMC template sources of EROs (and are labelled ERO in Tables A.1 and A.3), 65 sources (including eight in the LMC) are EROs with MIR spectra (and are labelled eroS in these tables), 110 sources (including two in the direction of the SMC, and 26 in the direction of the LMC) are candidate EROs based on the fitting of the SEDs (and are labelled eroP in these tables), and the remaining four are classified as C-rich non-ERO sources (and are labelled sedC in these tables). The O-rich sources appear to be a mixture of O-rich AGB and P-AGB stars, HII regions, planetary nebulae and YSOs (and are labelled sedO in these tables).

Table 2 shows the results for the C-stars and Table C.1 for the O-stars. Only the first entries are shown, and the full tables are available at the CDS. Displayed are the adopted distance and reddening and the results of fitting the SEDs. The last column shows the total MLR, which assumes spherical symmetry of the CSE, a dust-to-gas (DTG) ratio of 0.005, and a CSE expansion velocity of $10 \mathrm{~km} \mathrm{~s}^{-1}$ for every star.
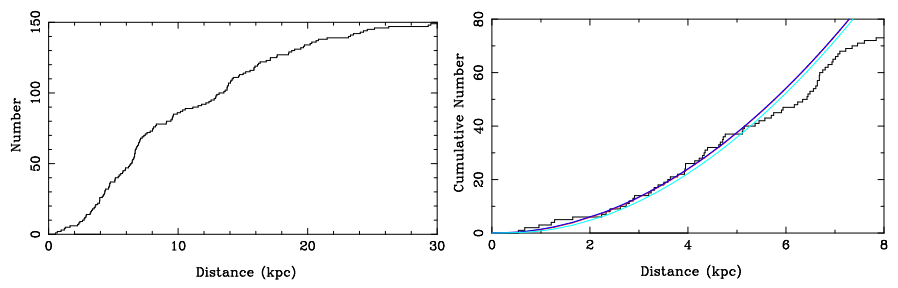

Fig. 5. Cumulative number distribution of ERO (candidates) versus distance. Right-hand panel: is a zoom, and it shows model predictions for combinations of local space density and vertical scale height of $1.6 \mathrm{kpc}^{-3}, 150 \mathrm{pc}$ (dark blue); $0.8 \mathrm{kpc}^{-3}, 300 \mathrm{pc}$ (red); and $0.30 \mathrm{kpc}^{-3}$, $800 \mathrm{pc}$ (light blue), respectively.

Figure 5 shows the cumulative number distributions of ERO (candidates) versus distance up to $30 \mathrm{kpc}$ on the left, and up to $8 \mathrm{kpc}$ on the right. When the number density of stars is assumed to depend exponentially on the height above the Galactic plane the number of stars within a certain radius can be calculated analytically, see Eq. (19) in Groenewegen et al. (1992), while Eq. (20) in that paper can be used to determine the scale height. The right-hand panel of Fig. 5 shows some models for different scale heights $(H)$ and local space densities $\left(\rho_{0}\right)$. The number of objects scales to first order with $\left(H \cdot \rho_{0}\right)$ and the three models that show a very similar behaviour all have $H \cdot \rho_{0}=0.24 \mathrm{kpc} \mathrm{kpc}^{-3}$. Groenewegen et al. (1992). based on a very small number of EROs derived $H=195 \pm 20 \mathrm{pc}$, and $H \cdot \rho_{0}=0.23-0.27 \mathrm{kpc} \mathrm{kpc}^{-3}$.

The predicted number of stars versus distance suggests that the sample of EROs may be complete up to $5 \mathrm{kpc}$. There are 36 stars in a cylinder around the Sun with this distance with a total estimated MLR of $4.1 \times 10^{-4} M_{\odot} \mathrm{yr}^{-1}$ (or $5.2 \times$ $10^{-6} M_{\odot} \mathrm{yr}^{-1} \mathrm{kpc}^{-2}$ ) and an estimated scale height of $180 \mathrm{pc}$. The average MLR is this sample is $1.1 \times 10^{-5} M_{\odot} \mathrm{yr}^{-1}$. As the DTG ratio is an assumed quantity (1/200), a more certain number is the dust-production rate (DPR) which is $2.0 \times$ $10^{-6} M_{\odot} \mathrm{yr}^{-1}$ (or $2.6 \times 10^{-8} M_{\odot} \mathrm{yr}^{-1} \mathrm{kpc}^{-2}$ ). To estimate an uncertainty on these numbers, Monte Carlo calculations were performed generating samples with other distances and MLRs, assuming Gaussian distributions with a width of 0.3 mag in $M_{\text {bol }}$ (which leads to a change in distance and in MLR), a condensation temperature taking the estimated error from Table 2 (with a minimum of $50 \mathrm{~K}$ ), and an optical depth taking the estimated error from Table 2. The $2.7 \%, 97.3 \%$, and $50 \%$ percentiles (corresponding to $\pm 2 \sigma$ in a Gaussian distribution and the median) indicate a number of stars of $37\left(33-41 ; 0.4-0.5 \mathrm{kpc}^{-2}\right)$, a cumulative MLR of $4.0\left(3.3-6.0 \times 10^{-4} M_{\odot} \mathrm{yr}^{-1}\right)$, and a $H=190(156-224 \mathrm{pc})$. Changing the limiting distance to $3 \mathrm{kpc}$ has some impact on the estimated DPR per unit surface area from $2.6\left(2.1-3.8 \times 10^{-8} M_{\odot} \mathrm{yr}^{-1} \mathrm{kpc}^{-2}\right)$ to $2.3(1.7-3.0 \times$ $\left.10^{-8} M_{\odot} \mathrm{yr}^{-1} \mathrm{kpc}^{-2}\right)$. The number of stars is reduced to the range 13 to 18 with a median of 16 . All (dust) MLRs quoted above are based on an average expansion velocity of $10 \mathrm{~km} \mathrm{~s}^{-1}$. If this were $15 \mathrm{~km} \mathrm{~s}^{-1}$ (as assumed in e.g. Jura \& Kleinmann 1989), all MLRs would increase by a factor of 1.5 .

The cumulative mass-loss return of the 45 ERO sources in the LMC is $1.0 \times 10^{-3} M_{\odot} \mathrm{yr}^{-1}$ (or $5.0 \times 10^{-6} M_{\odot} \mathrm{yr}^{-1}$ in dust). Thirty-three were modelled by Nanni et al. (2019), finding a total MLR of $7.8 \times 10^{-3} M_{\odot} \mathrm{yr}^{-1}$ (and $3.3 \times 10^{-6} M_{\odot} \mathrm{yr}^{-1}$ in dust $)^{16}$ and implying an average gas-to-dust ratio of 240 . Here we find $7.3 \times 10^{-3} M_{\odot} \mathrm{yr}^{-1}$ (or $3.7 \times 10^{-6} M_{\odot} \mathrm{yr}^{-1}$ in dust) for that sub-sample, which is in good agreement. What is interesting

${ }^{16}$ Using the J1000 set of models in Nanni et al. (2019). 
and in highlighting, again, the importance of the EROs is the impact of the only 12 stars not included in the study by Nanni et al. (2019). The total dust return by C-stars for the entire LMC is $16.0 \times 10^{-6} M_{\odot} \mathrm{yr}^{-1}$ (J1000 models; Nanni et al. 2019) from 8239 stars, of which $82 \%\left(13.1 \times 10^{-6} M_{\odot} \mathrm{yr}^{-1}\right)$ are by the $16 \%$ (1332) classified as X-stars. The sub-sample of 33 stars $(0.4 \%)$ already contributes $21 \%$ to the total dust return. Adding the other 12 stars $(0.15 \%)$ augments the total dust return by $8 \%$ to about $17.3 \times 10^{-6} M_{\odot} \mathrm{yr}^{-1}$.

\subsection{The nature of the EROs}

Although the C-rich EROs are thought to be major contributors to the dust and mass return of AGB stars to the ISM the nature of these objects is not fully understood. Many are clearly pulsating with large amplitudes and are LPVs. They follow a well-defined PL relation up to about 1260 days (see Fig. C.1). These objects definitely show the characteristics of AGB evolution. However, there are also objects that are classified as EROs based on the SEDs and MIR spectra that show no clear evidence for pulsation or variability, or with different properties.

This was first recognised in Sloan et al. (2016) where it is remarked that some of the embedded sources are relatively nonvariable and that some have relatively blue colours (compared to other embedded sources) at shorter wavelengths and that this can be interpreted as the central star revealing itself. They conclude that some deeply embedded stars may be evolving off of the AGB and/or they may have non-spherical dust geometries.

One of the parameters derived from the SED fitting is the temperature at the inner radius. In most cases, a standard value (800-1200 K, consistent with the condensation temperature of amorphous carbon dust) is sufficient to fit the data. However, for a non-negligible fraction of objects, a lower value has to be adopted, and this can be due to non-spherical dust geometries, or a spherical shell that expands, consistent with the drop in MLR when the AGB star evolves into the P-AGB phase. Of the 133 objects with a condensation temperature consistent with $800 \mathrm{~K}$ or more, 116 show a plausible pulsation period, and only $12 \%$ show no obvious variability or a period longer than 1300 days. For one-third of the sample (64 stars), a lower dust temperature at the inner radius is inferred of which $73 \%$ show no obvious variability or a period longer than 1300 days. This implies that lower temperatures at the inner radius are found for a nonnegligible number of objects and that these show, on average, less pronounced variability.

However, one issue with the interpretation of some of these stars evolving from the AGB into the P-AGB phase is the timescale. To investigate this further the SEDs and MIR spectra were calculated for two objects under the assumption that the MLR drops abruptly to zero and that the CSE then expands at a velocity of $10 \mathrm{~km} \mathrm{~s}^{-1}$. The results are shown in Fig. 6. The models in Fig. 6 were calculated for an effective temperatures typical for AGB stars (2600-2800 K). The P-AGB models of Miller Bertolami (2016) indicate that the effective temperature of stars with an initial mass of 2 and $3 M_{\odot}$ is about $3800 \mathrm{~K}$ at an envelope mass of $0.01 M_{\odot}$. Similar calculation were done for $T_{\text {eff }}=3800 \mathrm{~K}$ and are shown in Fig. D.1. The differences are small. The change in effective temperature at that phase of the evolution is also small, $0.22-0.52 \mathrm{~K} \mathrm{yr}^{-1}$. A first indication that the central star becomes slightly visible is present already after the order of 20-30 yr. When the dynamical time increases the central star becomes increasingly visible, until after about $500 \mathrm{yr}$ one has the classical SED of a P-AGB star with a double-peaked
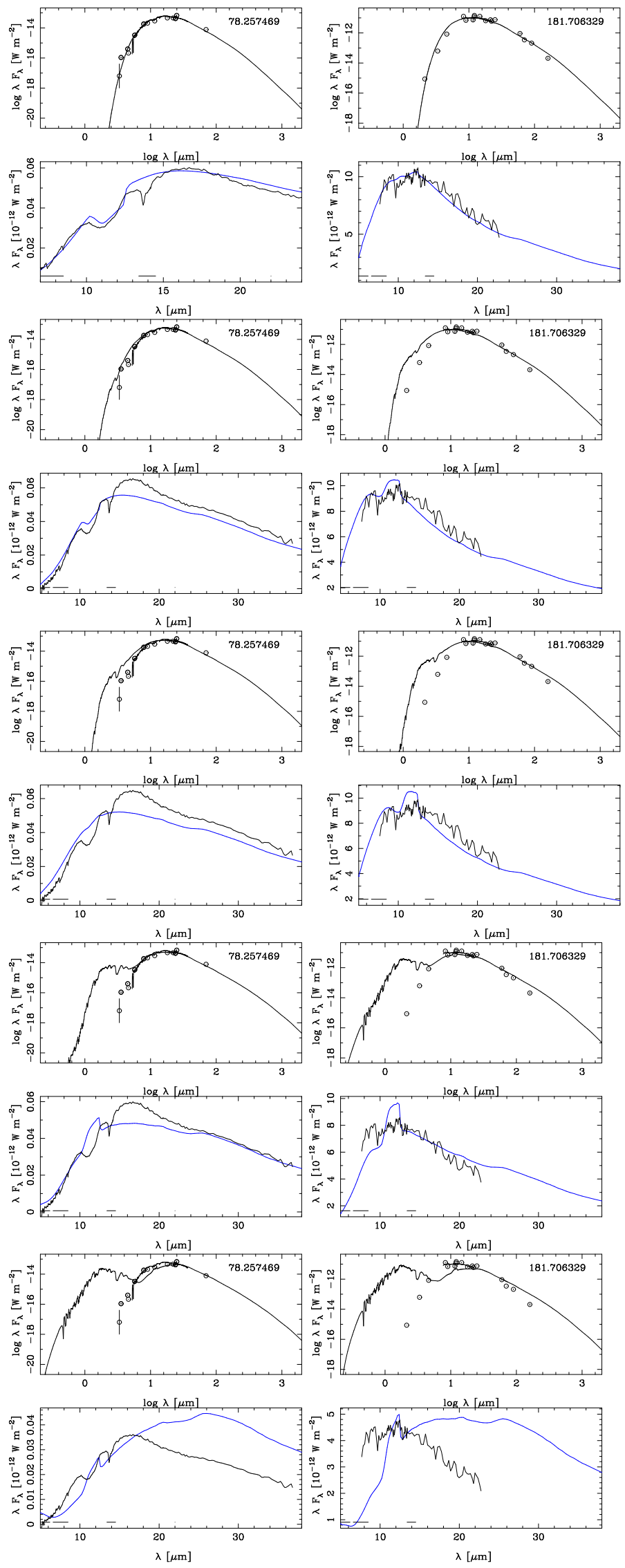

Fig. 6. Predicted SEDs and MIR spectra as a function time for two stars (left-hand and right-hand panels) when the MLR stops abruptly and the CSE expands at $10 \mathrm{~km} \mathrm{~s}^{-1}$. From top to bottom the SED at $t=0$, and after $30,50,200$, and $500 \mathrm{yr}$. 
SED. Important here is that the MIR bump remains bright and red, and so any selection of a sample based on MIR colours and magnitudes would be relatively insensitive to the expanding shell. One would therefore expect more objects in the sample of (candidate) EROs which show hints of a double-peaked SED, and this is not the case.

Recently Dell'Agli et al. (2021) propose that binary interaction mechanisms that involve common envelope evolution (CEE) could be a possible explanation, and that these stars could possibly hide binaries with orbital periods of the order of days. Their main argument is that single-star stellar evolution models combined with dust formation models could not produce the location of the EROs in certain colour-colour diagrams, and that this implies MLRs of $1-2 \times 10^{-4} M_{\odot} \mathrm{yr}^{-1}$ or larger. A binary scenario involving CEE might trigger the amount of dust to produce the observed colours. For the 11 stars in Table 1 in Dell'Agli et al. (2021), MLRs of $(0.7-3.3) \times 10^{-5} M_{\odot} \mathrm{yr}^{-1}$ are found for nine in the present study, that is significantly lower than $10^{-4} M_{\odot} \mathrm{yr}^{-1}$ (for our choice for the DTG ratio and expansion velocity). For SSID $125(\mathrm{RA}=82.684006)$ and SSID $190(\mathrm{RA}=87.249886)$, very large MLRs of 1.0 and $1.8 \times 10^{-4} M_{\odot} \mathrm{yr}^{-1}$ were indeed derived, respectively. The MLR in the latter source is the largest for all (candidate) EROs in the MCs, and only two show larger MLRs in our Galaxy, namely $2.1 \times 10^{-4} M_{\odot} \mathrm{yr}^{-1}$ $(\mathrm{RA}=283.812347)$, and $3.1 \times 10^{-4} M_{\odot} \mathrm{yr}^{-1}(\mathrm{RA}=328.768372)$.

In the sample of EROs in the MCs that define the PL relation, two objects were excluded as their periods (1884 and 3434 days but with large uncertainty) and luminosity did not match the relation. Similarly, among the Galactic ERO candidates, there are a few sources with (uncertain) periods in the range of 2000-5000 days where the PL relation was not applied. The longest period for which it was applied was about 2600 days. For longer periods, the implied luminosity would no longer be compatible with the AGB $\left(L \gtrsim 100000 L_{\odot}\right)$. For a few stars with shorter periods (1000-2000 days) located close to the Galactic plane, the PL relation is also unlikely to be valid. The implied luminosities from the PL relation are compatible with the AGB, but they lead to large distances $(\gtrsim 20 \mathrm{kpc})$ that imply large reddenings $\left(A_{V} \gtrsim 10 \mathrm{mag}\right)$ that are incompatible with the observed SEDs that show less extinction.

In summary, the nature of the EROs remain uncertain. Many show properties that are consistent with the properties expected for evolved AGB stars, but a significant fraction of them do not. The P-AGB channel may apply to some, but the time evolution of an expanding (spherical) shell would predict more objects with a classical double-peaked SED. The CEE channel is interesting, but it will be hard to prove the predicted binary period of the order of days. The derived MLRs are in general lower than predicted, although this depends on the assumed DTG ratio and expansion velocity, but in addition to the adopted MLR formalism in the stellar evolution models, which Dell' Agli et al. (2021) acknowledge might be too high. The effect of non-spherical CSEs is also a realistic option that needs to be investigated. Although challenging, high angular resolution observations in the MIR and the mm (with ALMA) might shed light on the morphology of the CSE. For an typical ERO at a $3 \mathrm{kpc}$ distance, the inner dust radius is predicted to be at about 10 mas, while the total CSE is of order $10^{\prime \prime}$.

\subsection{MIR spectra of mass-losing carbon stars as a tracer of interstellar extinction}

As part of the fitting of the SEDs and MIR spectra, the observed photometry and spectra were corrected for IS extinc- tion, including, in the MIR regime, the local ISM model of Chiar \& Tielens (2006), using a ratio of $A_{V} / A_{K}=0.118$ to scale it to the adopted reddening law of Cardelli et al. (1989) with the improvements by O'Donnell (1994) from the UV to the NIR in $\mathrm{MoD}$ (Groenewegen 2012). In this model, the extinction is smallest near $7.5 \mu \mathrm{m}\left(A_{7.5 \mu \mathrm{m}} / A_{K}=0.38\right)$ and has a peak at the silicate feature $\left(A_{9.8 \mathrm{um}} / A_{K} \approx 1.0\right)$. In other words, for $A_{V} \approx 8.5$ the extinction becomes $A_{9.8 \mu \mathrm{m}} \approx 1.0$, which should be noticeable in an MIR spectrum.

Figure 7 shows this very clearly where the SEDs and MIR spectra are shown for three stars with estimated $A_{V} \mathrm{~s}$ (see Appendix $\mathrm{C}$ on how $A_{V}$ was determined) of 2.4, 4.3, and $5.7 \mathrm{mag}$, respectively, and with no correction. Not only is the IS $9.8 \mu \mathrm{m}$ feature very evident, but the MIR spectrum is better fit over the entire wavelength range. The difference in the SEDs in the upper panels appears quite small in the optical, and this is due to the fact that luminosity and optical depth were refitted in the models with no IS extinction.

The principle of using MIR spectra to trace (MIR) interstellar extinction works for every type of star of course but the highly mass-losing C-stars have an advantage as they are bright in the MIR and have no strong intrinsic features near $10 \mu \mathrm{m}$. The same three stars without mass loss would be 4.0-4.6 mag fainter in the $N$-band. The same would be the case when using normal (O-rich) red giants, while when using MIR brighter masslosing O-rich stars one would have to distinguish between the circumstellar and IS silicate features which would be extremely challenging.

\section{Final remarks}

The period analysis of the WISE/NEOWISE time series data of 1775 objects colour selected to include known C-rich objects with flat MIR continua or $\mathrm{SiC}$ in absorption and known Galactic O-rich AGB stars with pulsation periods over 1000 days, supplemented with 217 AGB stars in the MCs previously studied in the $K$-band, is presented. In addition, periods from the literature and in many cases a reanalysis of time series data from other surveys is presented. The SEDs and MIR spectra were modelled with a dust RT programme for a subset of 316 stars as well. The results are presented in the seven subsections of Sect. 6. This includes the detection of new C-rich EROs and new LPVs, of which 145 have periods $>1000$ days.

The nature of the C-rich EROs remains uncertain. Most of the objects in the MCs follow a PL relation and this relation was used to estimate the distance to the Galactic EROs with a period. However, a significant fraction of galactic sources show variability not consistent with an AGB nature (implausibly long periods or no variability). A P-AGB nature for some EROs is difficult to exclude, but given the lifetimes, one would expect a larger number of SEDs with double-peaked SEDs. A possibility is that the shape of some of the SEDs is linked to an aspherical CSE, which is possibly linked to binarity. High spatial resolution line and continuum observations in the MIR or (sub-)mm would be helpful to better characterise the CSE of some of these objects.

In general, distance determinations to these red sources are crucial as well as is investigating and understanding their nature, in particular for the ERO sources. The PL relation is the only available method at the moment, but this has clear limitations. The targets studied here are ideal candidates for an IR astrometric mission (Hobbs et al. 2021). Of the 150 (candidate) EROs in our Galaxy about $85 \%$ have $K<20 \mathrm{mag}$. Going to even slightly redder wavelengths would be even more advantageous as all Galactic ERO candidates have $L<14.3$ for example. 
M. A. T. Groenewegen: A WISE view on extreme AGB stars
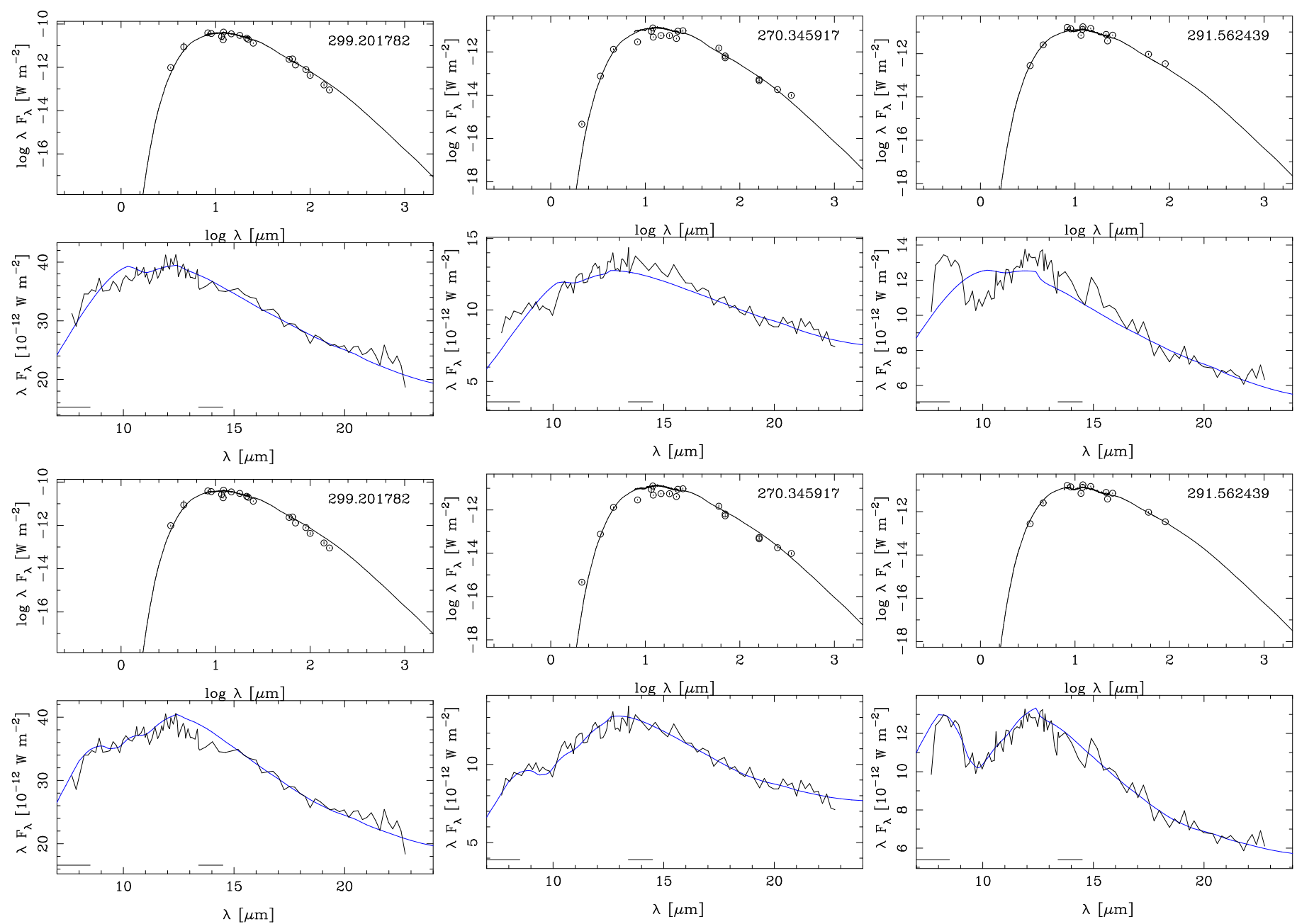

Fig. 7. Effect of $9.8 \mu \mathrm{m}$ silicate IS extinction feature. Top panels: fits to the SEDs and MIR spectra without IS extinction correction $\left(A_{V}=0\right)$, bottom panels: fits for three stars with, from left to right, $A_{V}=2.4,4.3$, and $5.7 \mathrm{mag}$, respectively. The luminosity and optical depth were refitted in the case of $A_{V}=0$ and that is the reason that the difference in the SEDs appears small.

Acknowledgements. The referee is thanked for pointing out the paper by Chen et al. (2020). This research has made use of the SIMBAD database, and the VizieR catalogue access tool, operated at CDS, Strasbourg, France (DOI: $10.26093 / \mathrm{cds} /$ vizier). The original description of the VizieR service was published in Ochsenbein et al. (2000). This publication makes use of data products from the Wide-field Infrared Survey Explorer, which is a joint project of the University of California, Los Angeles, and the Jet Propulsion Laboratory/California Institute of Technology, funded by the National Aeronautics and Space Administration. This publication also makes use of data products from NEOWISE, which is a project of the Jet Propulsion Laboratory/California Institute of Technology, funded by the Planetary Science Division of the National Aeronautics and Space Administration. Based on observations obtained with the Samuel Oschin 48-inch Telescope at the Palomar Observatory as part of the Zwicky Transient Facility project. ZTF is supported by the National Science Foundation under Grant No. AST-1440341 and a collaboration including Caltech, IPAC, the Weizmann Institute for Science, the Oskar Klein Center at Stockholm University, the University of Maryland, the University of Washington, Deutsches Elektronen-Synchrotron and Humboldt University, Los Alamos National Laboratories, the TANGO Consortium of Taiwan, the University of Wisconsin at Milwaukee, and Lawrence Berkeley National Laboratories. Operations are conducted by COO, IPAC, and UW.

\section{References}

Alfonso-Garzón, J., Domingo, A., Mas-Hesse, J. M., \& Giménez, A. 2012, A\&A, 548, A79

Aringer, B., Girardi, L., Nowotny, W., Marigo, P., \& Lederer, M. T. 2009, A\&A, 503, 913

Assef, R. J., Stern, D., Noirot, G., et al. 2018, ApJS, 234, 23
Bailer-Jones, C. A. L., Fouesneau, M., \& Andrae, R. 2019, MNRAS, 490, 5615 Bellm, E. C., Kulkarni, S. R., Graham, M. J., et al. 2019, PASP, 131, 018002 Benjamin, R. A., Churchwell, E., Babler, B. L., et al. 2003, PASP, 115, 953 Blum, R. D., Mould, J. R., Olsen, K. A., et al. 2006, AJ, 132, 2034 Boyer, M. L., Srinivasan, S., van Loon, J. T., et al. 2011, AJ, 142, 103 Boyer, M. L., Srinivasan, S., Riebel, D., et al. 2012, ApJ, 748, 40 Boyer, M. L., McQuinn, K. B. W., Barmby, P., et al. 2015, ApJ, 800, 51 Cardelli, J. A., Clayton, G. C., \& Mathis, J. S. 1989, ApJ, 345, 245 Chen, X., Wang, S., Deng, L., de Grijs, R., \& Yang, M. 2018, ApJS, 237, 28

Chen, X., Wang, S., Deng, L., et al. 2020, ApJS, 249, 18

Chiar, J. E., \& Tielens, A. G. G. M. 2006, ApJ, 637, 774

Cioni, M.-R. L., Clementini, G., Girardi, L., et al. 2011, A\&A, 527, A116

Contreras Peña, C., Lucas, P. W., Minniti, D., et al. 2017, MNRAS, 465, 3011

Csengeri, T., Urquhart, J. S., Schuller, F., et al. 2014, A\&A, 565, A75

Cutri, R. M. 2014, VizieR Online Data Catalog: II/328

Cutri, R. M., Skrutskie, M. F., van Dyk, S., et al. 2003, VizieR Online Data Catalog: II/246

Cutri, R. M., Skrutskie, M. F., van Dyk, S., et al. 2012, VizieR Online Data Catalog: II/281

de Grijs, R., \& Bono, G. 2015, AJ, 149, 179

de Grijs, R., Wicker, J. E., \& Bono, G. 2014, AJ, 147, 122

Dell'Agli, F., Marini, E., D’Antona, F., et al. 2021, MNRAS, 502, L35 DENIS Consortium 2005, VizieR Online Data Catalog: II/263

Drake, A. J., Djorgovski, S. G., Mahabal, A., et al. 2009, ApJ, 696, 870

Drake, A. J., Graham, M. J., Djorgovski, S. G., et al. 2014, ApJS, 213, 9

Eden, D. J., Moore, T. J. T., Plume, R., et al. 2017, MNRAS, 469, 2163

Egan, M. P., Price, S. D., Kraemer, K. E., et al. 2003, VizieR Online Data Catalog: V/114

Elia, D., Molinari, S., Schisano, E., et al. 2017, MNRAS, 471, 100

Engels, D., \& Bunzel, F. 2015, A\&A, 582, A68 
Engels, D., Kreysa, E., Schultz, G. V., \& Sherwood, W. A. 1983, A\&A, 124, 123

Engels, D., Etoka, S., Gérard, E., \& Richards, A. 2015, in Why Galaxies Care about AGB Stars III: A Closer Look in Space and Time, eds. F. Kerschbaum, R. F. Wing, \& J. Hron, ASP Conf. Ser., 497, 473

Etoka, S., \& Diamond, P. 2007, in Why Galaxies Care About AGB Stars: Their Importance as Actors and Probes, eds. F. Kerschbaum, C. Charbonnel, \& R. F. Wing, ASP Conf. Ser., 378, 297

Evans, A., Gehrz, R. D., Woodward, C. E., et al. 2020, MNRAS, 493, 1277

Ferreira Lopes, C. E., Cross, N. J. G., Catelan, M., et al. 2020, MNRAS, 496, 1730

Flesch, E. W. 2015, PASA, 32, e010

Gaia Collaboration (Brown, A. G. A., et al.) 2018, A\&A, 616, A1

Gaia Collaboration (Brown, A. G. A., et al.) 2021a, A\&A, 649, A1

Gaia Collaboration (Klioner, S. A., et al.) 2021b, A\&A, 649, A9

Gattano, C., Andrei, A. H., Coelho, B., et al. 2018, A\&A, 614, A140

Goldman, S. R., van Loon, J. T., Zijlstra, A. A., et al. 2017, MNRAS, 465, 403

Goldman, S. R., van Loon, J. T., Gómez, J. F., et al. 2018, MNRAS, 473, 3835

Green, G. M., Schlafly, E., Zucker, C., Speagle, J. S., \& Finkbeiner, D. 2019, ApJ, 887, 93

Groenewegen, M. A. T. 2004, A\&A, 425, 595

Groenewegen, M. A. T. 2012, A\&A, 543, A36

Groenewegen, M. A. T., \& Sloan, G. C. 2018, A\&A, 609, A114

Groenewegen, M. A. T., de Jong, T., van der Bliek, N. S., Slijkhuis, S., \& Willems, F. J. 1992, A\&A, 253, 150

Groenewegen, M. A. T., Whitelock, P. A., Smith, C. H., \& Kerschbaum, F. 1998, MNRAS, 293, 18

Groenewegen, M. A. T., Barlow, M. J., Blommaert, J. A. D. L., et al. 2012, A\&A 543, L8

Groenewegen, M. A. T., Nanni, A., Cioni, M. R. L., et al. 2020, A\&A, 636, A48

Gruendl, R. A., \& Chu, Y.-H. 2009, ApJS, 184, 172

Gruendl, R. A., Chu, Y. H., Seale, J. P., et al. 2008, ApJ, 688, L9

Guo, S., Qi, Z., Liao, S., et al. 2018, A\&A, 618, A144

Gustafsson, B., Edvardsson, B., Eriksson, K., et al. 2008, A\&A, 486, 951

Gutermuth, R. A., \& Heyer, M. 2015, AJ, 149, 64

Hackstein, M., Fein, C., Haas, M., et al. 2015, Astron. Nachr., 336, 590

Hauschildt, P. H., Allard, F., \& Baron, E. 1999, ApJ, 512, 377

Heinze, A. N., Tonry, J. L., Denneau, L., et al. 2018, AJ, 156, 241

Herman, J., \& Habing, H. J. 1985, Phys. Rep., 124, 255

Herschel Point Source Catalogue Working Group (Marton, G., et al.) 2017, VizieR Online Data Catalog: VIII/106

Hobbs, D., Brown, A., Høg, E., et al. 2021, Exp. Astron., 51, 783

Houck, J. R., Roellig, T. L., van Cleve, J., et al. 2004, ApJS, 154, 18

Höfner, S., \& Olofsson, H. 2018, A\&ARv, 26, 1

Hyland, A. R. 1974, in Galactic Radio Astronomy, eds. F. J. Kerr, \& S. C. Simonson, IAU Symp., 60, 439

Ishihara, D., Onaka, T., Kataza, H., et al. 2010, A\&A, 514, A1

Ivezić, Ž., Nenkova, M., \& Elitzur, M. 1999, Astrophysics Source Code Library [record ascl:9911.001]

Jayasinghe, T., Kochanek, C. S., Stanek, K. Z., et al. 2018, MNRAS, 477, 3145

Jiménez-Esteban, F. M., García-Lario, P., Engels, D., \& Manchado, A. 2006, A\&A, 458, 533

Joint IRAS Science Working Group 1986, VizieR Online Data Catalog: II/125

Jones, T. J., Hyland, A. R., Caswell, J. L., \& Gatley, I. 1982, ApJ, 253, 208

Jones, T. J., Bryja, C. O., Gehrz, R. D., et al. 1990, ApJS, 74, 785

Jura, M., \& Kleinmann, S. G. 1989, ApJ, 341, 359

Justtanont, K., Barlow, M. J., Blommaert, J., et al. 2015, A\&A, 578, A115

Kamizuka, T., Nakada, Y., Yanagisawa, K., et al. 2020, ApJ, 897, 42

Kato, D., Nagashima, C., Nagayama, T., et al. 2007, PASJ, 59, 615

Kato, D., Ita, Y., Onaka, T., et al. 2012, AJ, 144, 179

Kerschbaum, F., Groenewegen, M. A. T., \& Lazaro, C. 2006, A\&A, 460, 539

Kiss, L. L., Derekas, A., Szabó, G. M., Bedding, T. R., \& Szabados, L. 2007, MNRAS, 375, 1338

Kochanek, C. S., Shappee, B. J., Stanek, K. Z., et al. 2017, PASP, 129, 104502

Kwok, S., Volk, K., \& Bidelman, W. P. 1997, ApJS, 112, 557

Lallement, R., Capitanio, L., Ruiz-Dern, L., et al. 2018, A\&A, 616, A132

Le Bertre, T. 1992, A\&AS, 94, 377

Le Bertre, T. 1993, A\&AS, 97, 729

Lebouteiller, V., Barry, D. J., Spoon, H. W. W., et al. 2011, ApJS, 196, 8

Lenz, P., \& Breger, M. 2005, Commun. Asteroseismol., 146, 53

Lewis, B. M. 2002, ApJ, 576, 445

Lucas, P. W., Hoare, M. G., Longmore, A., et al. 2008, MNRAS, 391, 136
Lyke, B. W., Higley, A. N., McLane, J. N., et al. 2020, ApJS, 250, 8

Macri, L. M., Ngeow, C.-C., Kanbur, S. M., Mahzooni, S., \& Smitka, M. T. 2015, AJ, 149, 117

Mainzer, A., Bauer, J., Grav, T., et al. 2011, ApJ, 731, 53

Mainzer, A., Bauer, J., Cutri, R. M., et al. 2014, ApJ, 792, 30

Masci, F. J., Laher, R. R., Rusholme, B., et al. 2019, PASP, 131, 018003

Matsuura, M., Barlow, M. J., Zijlstra, A. A., et al. 2009, MNRAS, 396, 918

Meixner, M., Gordon, K. D., Indebetouw, R., et al. 2006, AJ, 132, 2268

Menzies, J. W., Whitelock, P. A., Feast, M. W., \& Matsunaga, N. 2019, MNRAS, 483, 5150

Miller Bertolami, M. M. 2016, A\&A, 588, A25

Minniti, D., Lucas, P. W., Emerson, J. P., et al. 2010, New Astron., 15, 433

Minniti, D., Lucas, P., \& VVV team 2017, VizieR Online Data Catalog: II/348

Molinari, S., Schisano, E., Elia, D., et al. 2016, A\&A, 591, A149

Moshir, M. 1990, IRAS Faint Source Catalogue, version 2.0

Nakashima, J.-I., Jiang, B. W., Deguchi, S., Sadakane, K., \& Nakada, Y. 2000, PASJ, 52, 275

Nanni, A., Groenewegen, M. A. T., Aringer, B., et al. 2019, MNRAS, 487, 502

Neugebauer, G., \& Leighton, R. B. 1969, Two-micron Sky Survey. A Preliminary Catalogue (Washington: NASA)

Ochsenbein, F., Bauer, P., \& Marcout, J. 2000, A\&AS, 143, 23

O’Donnell, J. E. 1994, ApJ, 422, 158

Olivier, E. A., Whitelock, P., \& Marang, F. 2001, MNRAS, 326, 490

Petrosky, E., Hwang, H.-C., Zakamska, N. L., Chandra, V., \& Hill, M. J. 2021, MNRAS, 503, 3975

Pojmanski, G. 2002, Acta Astron., 52, 397

Press, W. H., Teukolsky, S. A., Vetterling, W. T., \& Flannery, B. P. 1992, Numerical Recipes in FORTRAN. The Art of Scientific Computing (Cambridge: University Press)

Price, S. D., \& Walker, R. G. 1976, The AFGL Four Color Infrared Sky Survey: Catalog of Observations at 4.2, 11.0, 19.8 and 27.4 Micrometers (Interim Report Air Force Geophysics Lab)

Price, S. D., Smith, B. J., Kuchar, T. A., Mizuno, D. R., \& Kraemer, K. E. 2010, ApJS, 190, 203

Riebel, D., Boyer, M. L., Srinivasan, S., et al. 2015, ApJ, 807, 1

Seale, J. P., Meixner, M., Sewiło, M., et al. 2014, AJ, 148, 124

Shappee, B. J., Prieto, J. L., Grupe, D., et al. 2014, ApJ, 788, 48

Skiff, B. A. 2014, VizieR Online Data Catalog: B/mk

Skowron, D. M., Skowron, J., Udalski, A., et al. 2021, ApJS, 252, 23

Sloan, G. C., Kraemer, K. E., Price, S. D., \& Shipman, R. F. 2003, ApJS, 147, 379

Sloan, G. C., Kraemer, K. E., McDonald, I., et al. 2016, ApJ, 826, 44

Solarz, A., Bilicki, M., Gromadzki, M., et al. 2017, A\&A, 606, A39

Speck, A. K., Corman, A. B., Wakeman, K., Wheeler, C. H., \& Thompson, G. 2009, ApJ, 691, 1202

Spitzer Science Center 2009, VizieR Online Data Catalog: II/293

Suh, K.-W., \& Kim, H. Y. 2002, A\&A, 391, 665

Tonry, J. L., Denneau, L., Heinze, A. N., et al. 2018, PASP, 130, 064505

Uchiyama, M., \& Ichikawa, K. 2019, ApJ, 883, 6

Urago, R., Omodaka, T., Nagayama, T., et al. 2020, ApJ, 891, 50

Uttenthaler, S., McDonald, I., Bernhard, K., Cristallo, S., \& Gobrecht, D. 2019, A\&A, 622, A120

van Langevelde, H. J., van der Heiden, R., \& van Schooneveld, C. 1990, A\&A, 239, 193

Ventura, P., Karakas, A. I., Dell'Agli, F., et al. 2016, MNRAS, 457, 1456

Véron-Cetty, M. P., \& Véron, P. 2010, A\&A, 518, A10

Volk, K., \& Cohen, M. 1989, AJ, 98, 931

Volk, K., Kwok, S., \& Langill, P. P. 1992, ApJ, 391, 285

Werner, M. W., Roellig, T. L., Low, F. J., et al. 2004, ApJS, 154, 1

Whitelock, P., Menzies, J., Feast, M., et al. 1994, MNRAS, 267, 711

Whitelock, P. A., Feast, M. W., van Loon, J. T., \& Zijlstra, A. A. 2003, MNRAS, 342, 86

Whitelock, P. A., Feast, M. W., Marang, F., \& Groenewegen, M. A. T. 2006, MNRAS, 369, 751

Whitelock, P. A., Feast, M. W., \& Van Leeuwen, F. 2008, MNRAS, 386, 313

Wood, P. R. 1998, A\&A, 338, 592

Wood, P. R., Whiteoak, J. B., Hughes, S. M. G., et al. 1992, ApJ, 397, 552

Woźniak, P. R., Williams, S. J., Vestrand, W. T., \& Gupta, V. 2004, AJ, 128, 2965

Wright, E. L., Eisenhardt, P. R. M., Mainzer, A. K., et al. 2010, AJ, 140, 1868

Yamamura, I., Makiuti, S., Ikeda, N., et al. 2010, VizieR Online Data Catalog: II/298

Yanagisawa, K., Shimizu, Y., Okita, K., et al. 2019, PASJ, 71, 118 
M. A. T. Groenewegen: A WISE view on extreme AGB stars

\section{Appendix A: Basic data and results from the period analysis}

Tables A.1, A.2, A.3, A.4, A.5, A.6 contain the basic data and the results of the period analysis.

Table A.1. Sample of bona fide stellar sources with a period analysis based on WISE data, general information (first entries).

\begin{tabular}{|c|c|c|c|c|c|c|c|c|c|c|c|c|c|c|c|}
\hline $\begin{array}{r}\mathrm{Ra} \\
(\mathrm{deg}) \\
\end{array}$ & $\begin{array}{r}\text { Dec } \\
(\mathrm{deg})\end{array}$ & Identifiers & $\begin{array}{l}\text { Dist } \\
\left({ }^{\prime \prime}\right)\end{array}$ & ObjType & $\begin{array}{l}2 \mathrm{M} \\
\left({ }^{\prime \prime}\right)\end{array}$ & $\begin{array}{l}\text { GLP } \\
\left({ }^{\prime \prime}\right) \\
\end{array}$ & $\begin{array}{l}\text { Aka } \\
\left({ }^{\prime \prime}\right)\end{array}$ & $\begin{array}{l}\text { MSX } \\
\left({ }^{\prime \prime}\right)\end{array}$ & $\begin{array}{l}\text { MGL } \\
\left({ }^{\prime \prime}\right)\end{array}$ & $\begin{array}{l}\text { HGL } \\
\left({ }^{\prime \prime}\right)\end{array}$ & $\begin{array}{l}V_{\mathrm{b}} \\
\left(\mathrm{km} \mathrm{s}^{-1}\right)\end{array}$ & $\begin{array}{l}V_{\mathrm{r}} \\
\left(\mathrm{km} \mathrm{s}^{-1}\right)\end{array}$ & LRS & Spec. Type & Comment \\
\hline 19.463942 & 67.231448 & IRAS01144+6658 RAFGL190 & 0.22 & $\mathrm{C}$ & & & 1.1 & 1.7 & & & nd & nd & $\mathrm{U}$ & & \\
\hline 124.826307 & -21.737399 & IRAS08171-2134 RAFGL5250 & 0.30 & $\mathrm{C}$ & 0.3 & & 0.1 & & & & - & - & $\mathrm{U}$ & & \\
\hline 237.773837 & -56.890007 & IRAS15471-5644 & 0.20 & Can.C & 0.2 & & 1.2 & 0.9 & & & nd & nd & $\mathrm{U}$ & & \\
\hline 287.486914 & 9.447611 & IRAS19075+0921 RAFGL2333 & 0.03 & $\mathrm{C}$ & 0.0 & & 1.2 & 0.8 & & 1.6 & - & - & $\mathrm{U}$ & & \\
\hline 323.345002 & 56.743063 & IRAS21318+5631 RAFGL5625S & 0.51 & $\mathrm{C}$ & & & 1.1 & 0.5 & & & nd & nd & $\mathrm{U}$ & $\mathrm{C}$ & \\
\hline 349.802533 & 17.192628 & IRAS23166+1655 RAFGL3068 & 0.33 & $\mathrm{C}$ & 0.3 & & 0.7 & & & & - & - & & $\mathrm{C}$ & \\
\hline 89.161446 & -67.892776 & IRAS05568-6753 & 0.23 & $\mathrm{C}$ & & & 1.9 & 3.4 & & & - & - & & & \\
\hline 82.407959 & -72.831322 & ERO0529379 IRAS05305-7251 & 0.17 & $\mathrm{C}$ & & & 0.7 & 1.4 & & & - & - & & & $q(6)$ \\
\hline 87.608788 & -69.934212 & ERO0550261 IRAS05509-6956 & 0.16 & $\mathrm{C}$ & & & 0.8 & 0.7 & & & - & - & & & $\mathrm{q}(6)$ \\
\hline 79.701599 & -69.559563 & ERO0518484 IRAS05191-6936 & 0.30 & $\mathrm{C}$ & & & 1.5 & 3.1 & & & - & - & & & \\
\hline 87.249886 & -70.556229 & IRAS05495-7034 & 0.77 & AGB & & & 0.6 & & & & - & - & & & \\
\hline 75.631233 & -68.093285 & ERO0502315 SSTISAGE1CJ050231.47-680535.9 & 0.04 & AGB & & & 1.1 & 3.3 & & & - & - & & & \\
\hline 76.023376 & -68.394501 & ERO0504056 IRAS05042-6827 & 0.16 & $\mathrm{C}$ & & & 1.1 & & & & - & - & & & \\
\hline 81.419411 & -70.140877 & ERO0525406 IRAS05260-7010 & 0.15 & $\mathrm{C}$ & & & 0.4 & 2.3 & & & - & - & & & \\
\hline 78.257469 & -69.564110 & IRAS05133-6937 & 0.28 & $\mathrm{C}$ & & & 0.8 & & & & - & - & & & \\
\hline 79.548790 & -70.507469 & ERO0518117 IRAS05187-7033 & 0.12 & $\mathrm{C}$ & & & 0.7 & & & & - & - & & & \\
\hline 282.174774 & -2.841357 & IRAS18460-0254 OH30.1-0.7 RAFGL5535 & 0.06 & $\mathrm{OH} / \mathrm{IR}$ & & & 1.0 & 1.0 & & 1.2 & 78.5 & 118.9 & & & \\
\hline 282.859344 & -1.064581 & IRAS18488-0107 OH32.0-0.5 & 0.36 & $\mathrm{OH} / \mathrm{IR}$ & & & 0.6 & 0.8 & & 0.7 & 55.2 & 95.8 & A & & \\
\hline 283.092775 & -0.236713 & IRAS18498-0017 OH32.8-0.3 RAFGL5540 & 0.37 & $\mathrm{OH} / \mathrm{IR}$ & 0.4 & & 0.8 & 1.0 & & 1.5 & 45.6 & 76.0 & $\mathrm{H}$ & & \\
\hline 287.284637 & 8.276116 & GPSR042.309-0.133 & 0.78 & Radio & & & 1.7 & 1.2 & & 0.3 & 42.3 & 75.3 & & & \\
\hline 23.463343 & 62.448162 & IRAS01304+6211 OH127.8-0.0 RAFGL230 & 0.19 & $\mathrm{OH} / \mathrm{IR}$ & 0.2 & & 0.2 & 0.2 & & & -65.2 & -43.0 & A & & \\
\hline 53.377491 & 60.335957 & IRAS03293+6010 OH141.7+3.5 RAFGL5097 & 0.61 & Mira & 0.1 & & 0.8 & & & & -69.1 & -44.9 & A & & \\
\hline 79.197746 & 45.567860 & IRAS05131+4530 RAFGL712 & 0.22 & $\mathrm{OH} / \mathrm{IR}$ & 0.2 & & & & & & nd & nd & $\mathrm{F}$ & & \\
\hline 111.101738 & -20.198791 & IRAS07222-2005 & 0.45 & Star & 0.4 & & 0.6 & 1.5 & & & 76.9 & 93.2 & $\mathrm{E}$ & M9: & \\
\hline 266.099701 & -31.927624 & IRAS17411-3154 RAFGL5379 & 4.13 & $\mathrm{OH} / \mathrm{IR}$ & & & & 0.7 & & 0.6 & -39.2 & -2.7 & & & \\
\hline 280.309784 & -6.250149 & IRAS18385-0617 OH26.2-0.6 & 0.22 & $\mathrm{OH} / \mathrm{IR}$ & 0.2 & & 2.0 & 1.3 & & 0.9 & 49.8 & 93.0 & & & \\
\hline 277.128905 & -9.970644 & IRAS18257-1000 OH21.5+0.5 & 0.00 & $\mathrm{OH} / \mathrm{IR}$ & & & 1.1 & 0.7 & & 1.8 & 97.6 & 134.0 & & & \\
\hline 51.285198 & 65.535362 & IRAS03206+6521 OH138.0+7.2 RAFGL5093 & 0.35 & $\mathrm{OH} / \mathrm{IR}$ & 0.4 & & 0.6 & & & & -46.4 & -27.6 & A & & \\
\hline 0.441592 & 62.748863 & IRAS23592+6228 & 2.27 & Star & 0.1 & & 0.9 & 1.4 & & & - & - & $\mathrm{F}$ & & \\
\hline 3.853554 & 54.906147 & IRAS00127+5437 & 0.21 & Star & 0.1 & & 0.2 & & & & - & - & $\mathrm{E}$ & & \\
\hline 4.963700 & 65.991821 & IRAS $00170+6542$ & 0.20 & Can.PAGB & 0.1 & & 1.2 & 1.5 & & & -65.0 & -37.6 & E & & \\
\hline
\end{tabular}

Notes. Column 16. References for extragalactic catalogues ('Q' marks probable QSOs, 'q' marks candidate QSOs). (1) Flesch (2015), (2) Bailer-Jones et al. (2019), (3) Guo et al. (2018), (4) Assef et al. (2018), (5) Gaia Collaboration (2021b), and (6) Solarz et al. (2017). None of the sources is listed in the QSO catalogues of Véron-Cetty \& Véron (2010), Gattano et al. (2018), and Lyke et al. (2020). The full table is available at the CDS. 


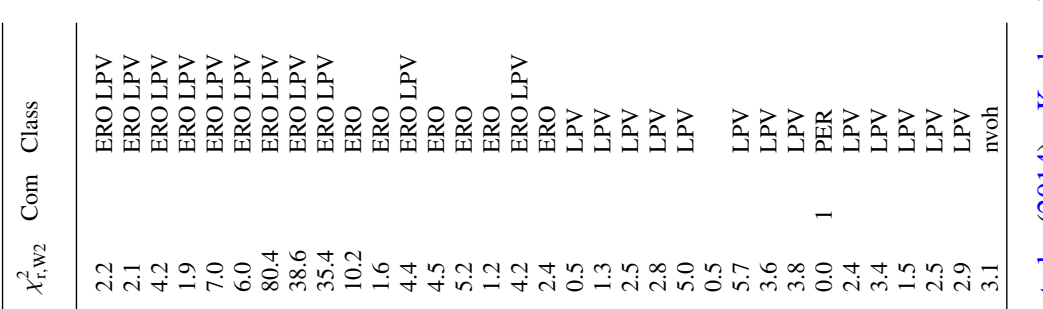

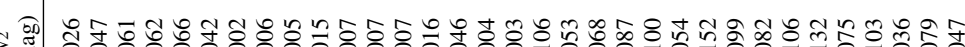
00000

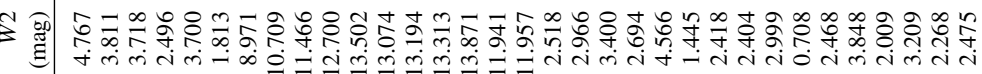

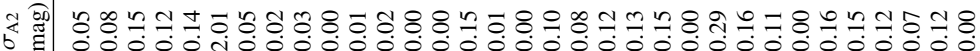

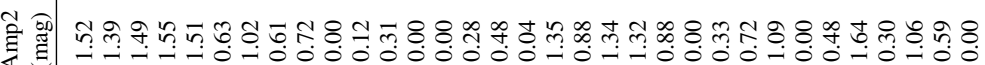

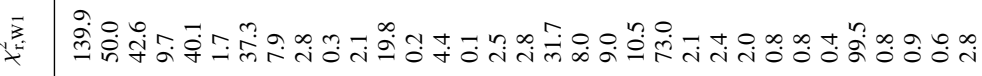
店热

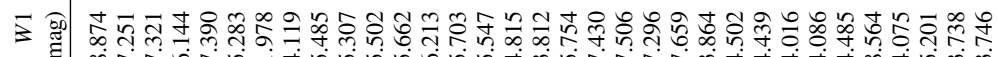
उ

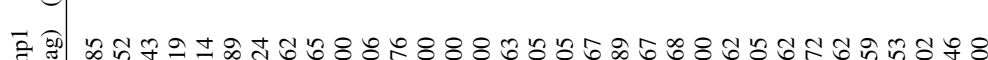

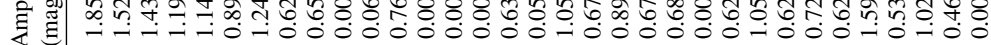

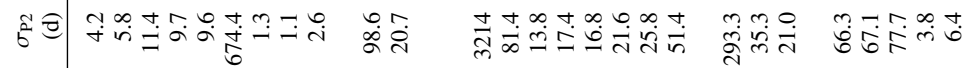

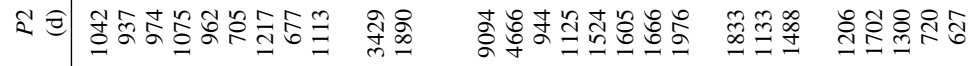

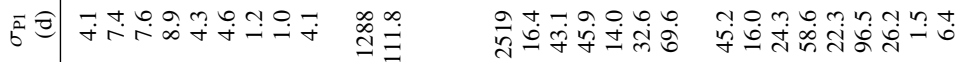

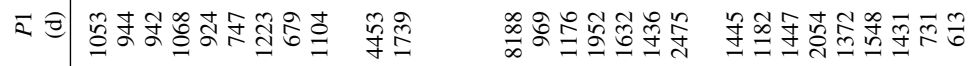

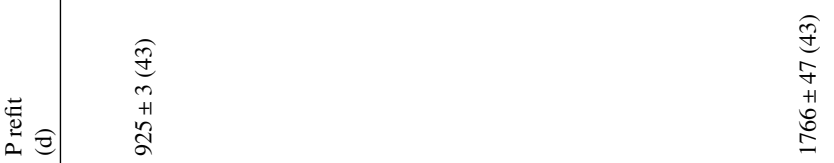

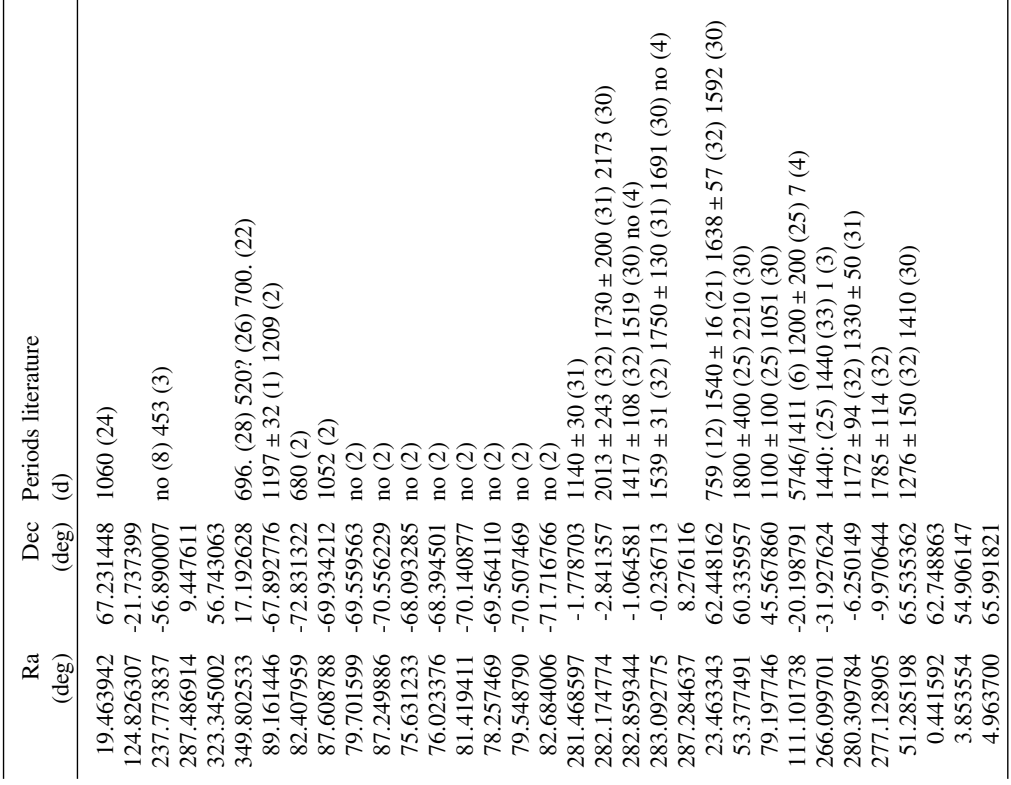

A145, page 16 of 31

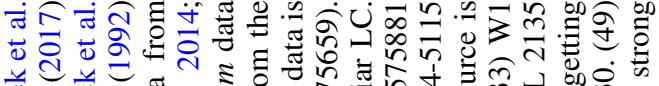

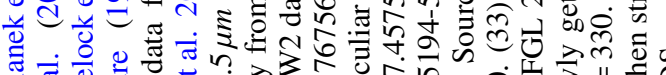

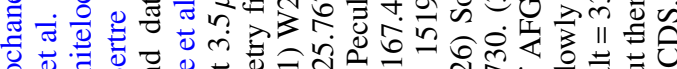

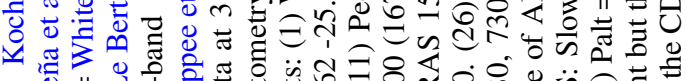

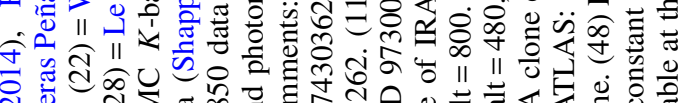

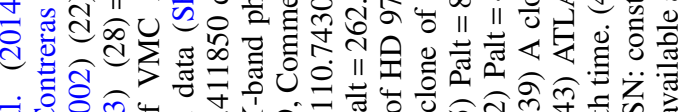

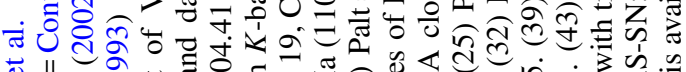

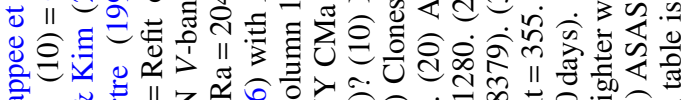

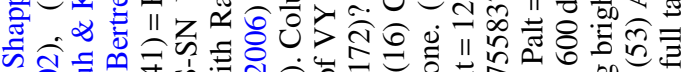

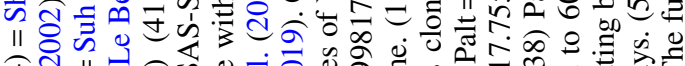

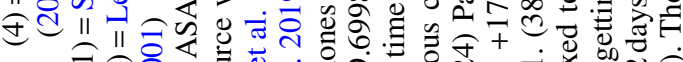

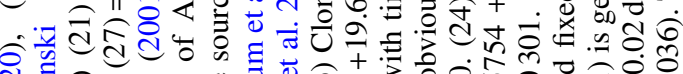

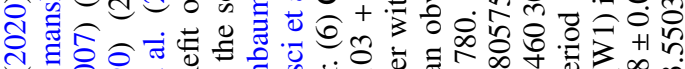

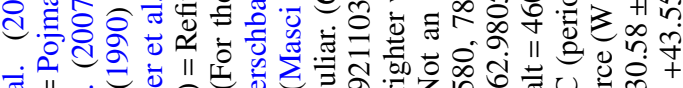
向

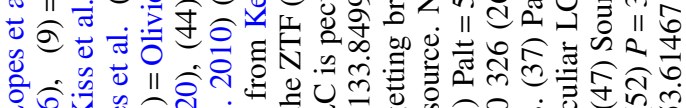

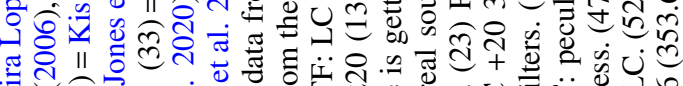

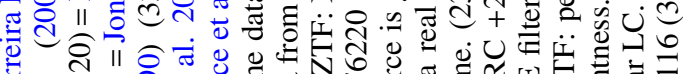

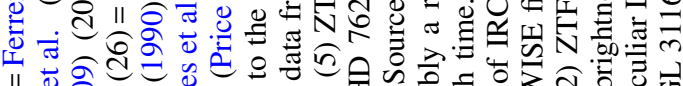

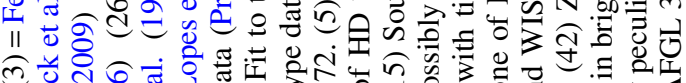

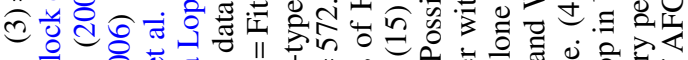
क人.

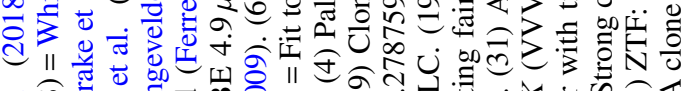

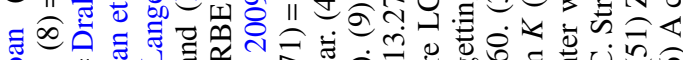
。

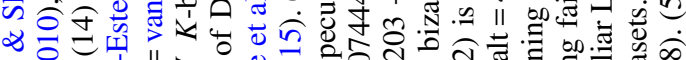

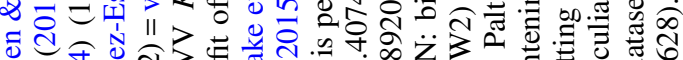

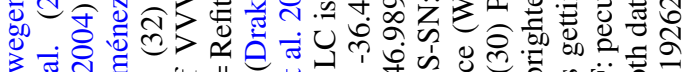

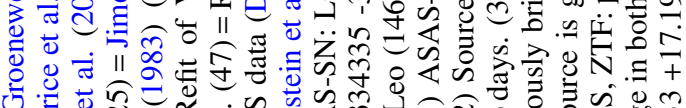

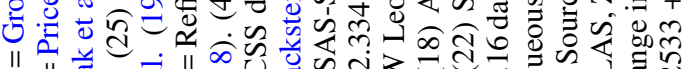

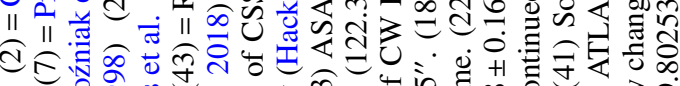

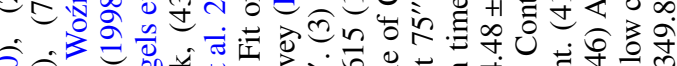

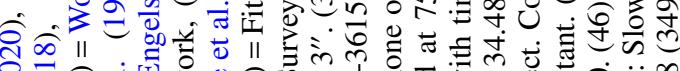

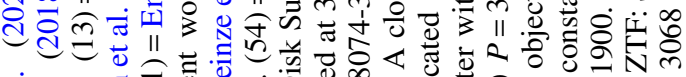

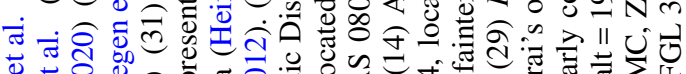

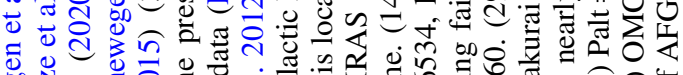

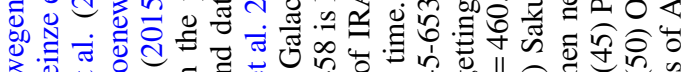

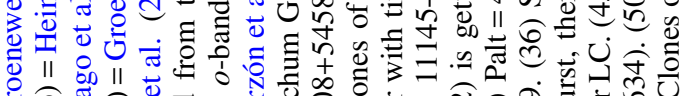

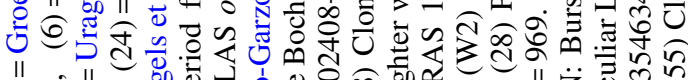

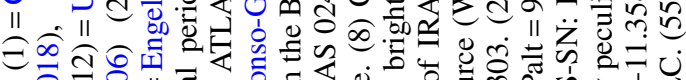

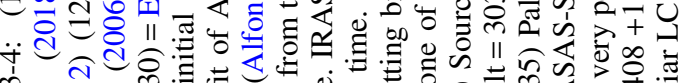

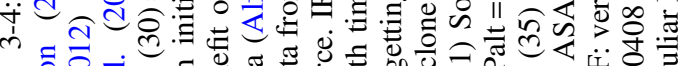

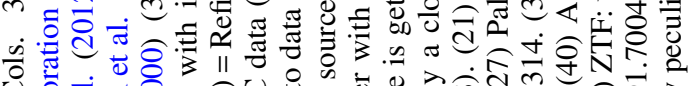

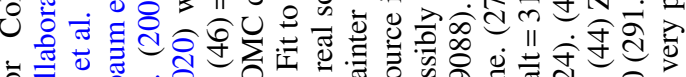

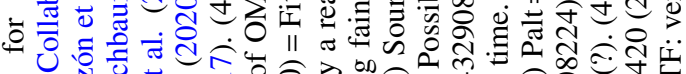

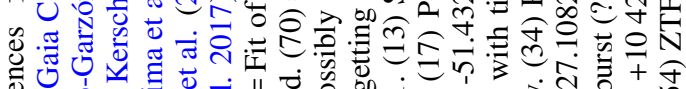

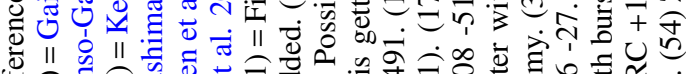

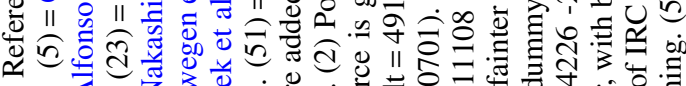

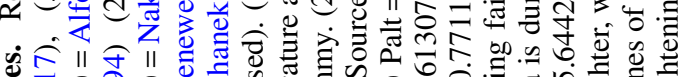

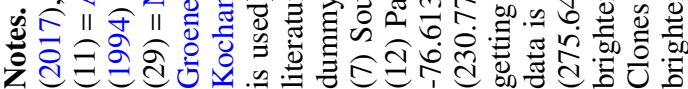


M. A. T. Groenewegen: A WISE view on extreme AGB stars

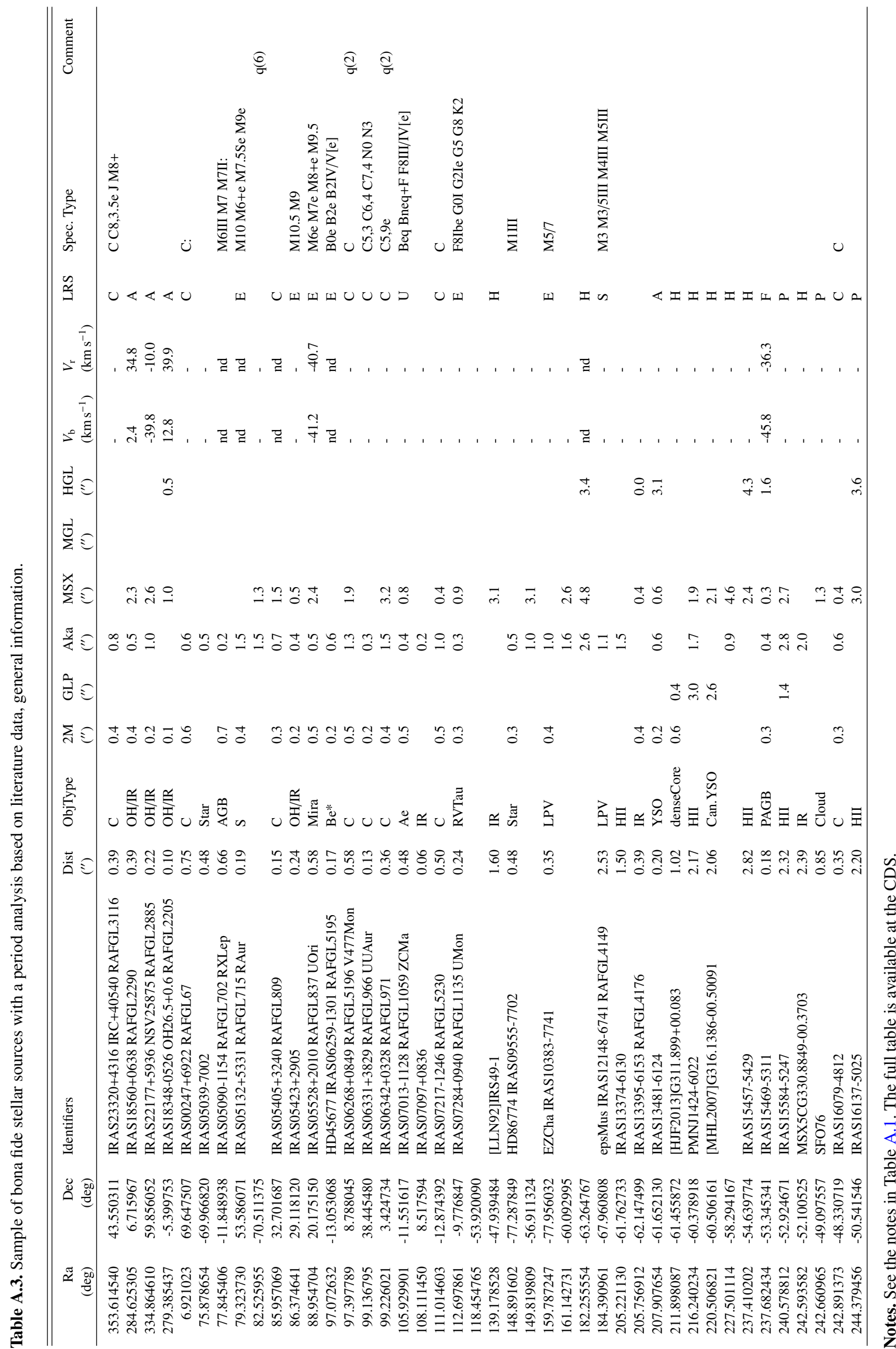




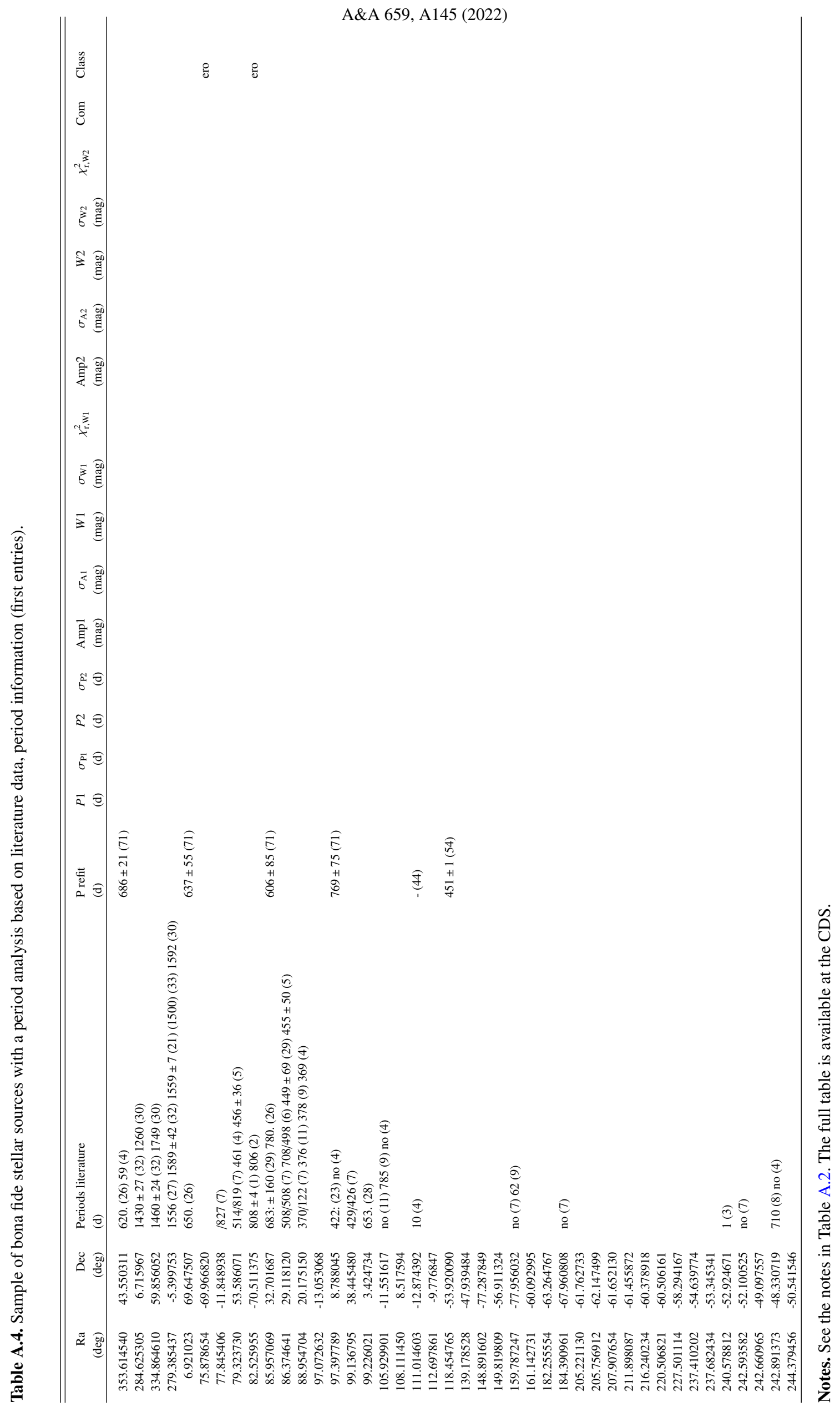




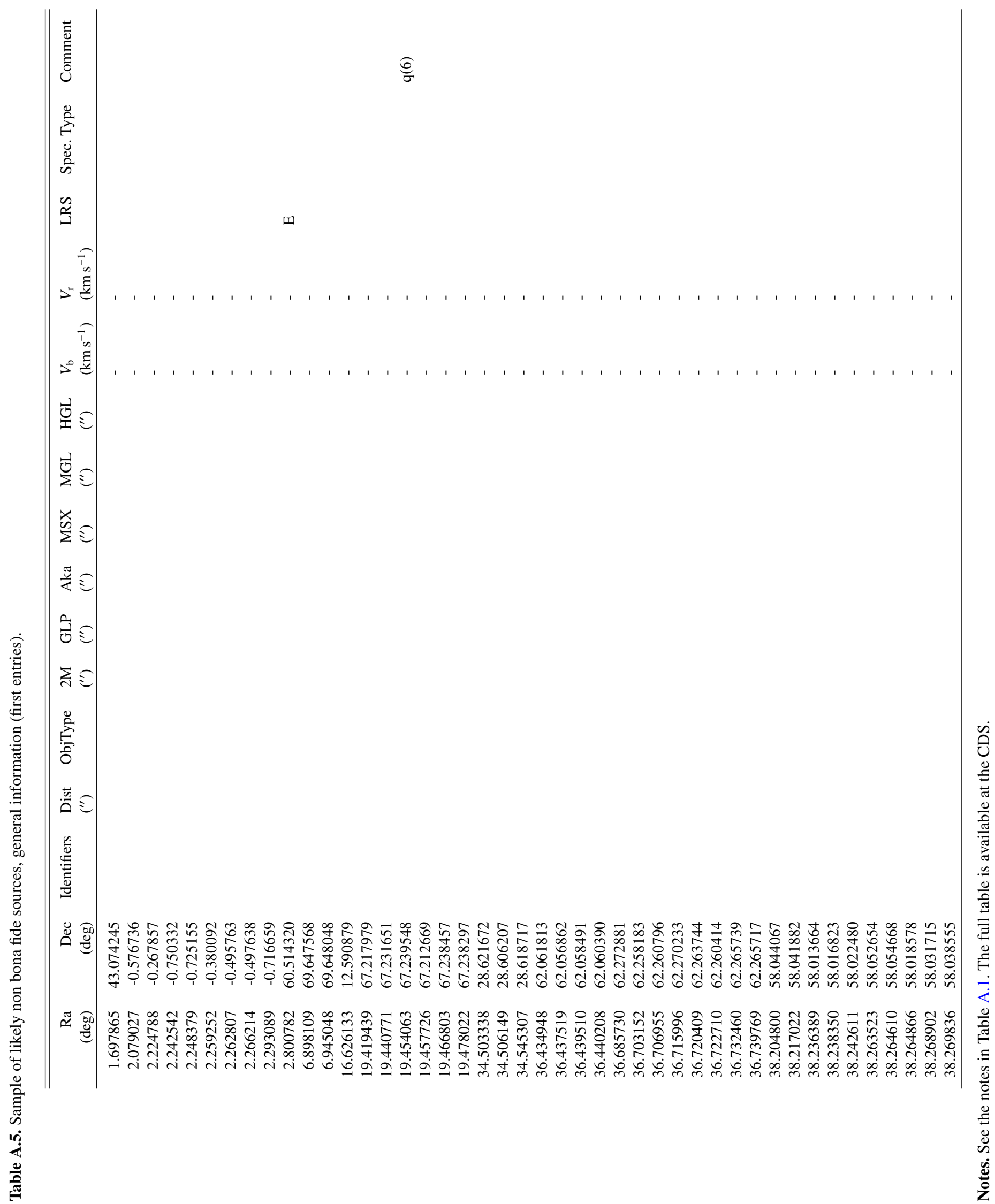




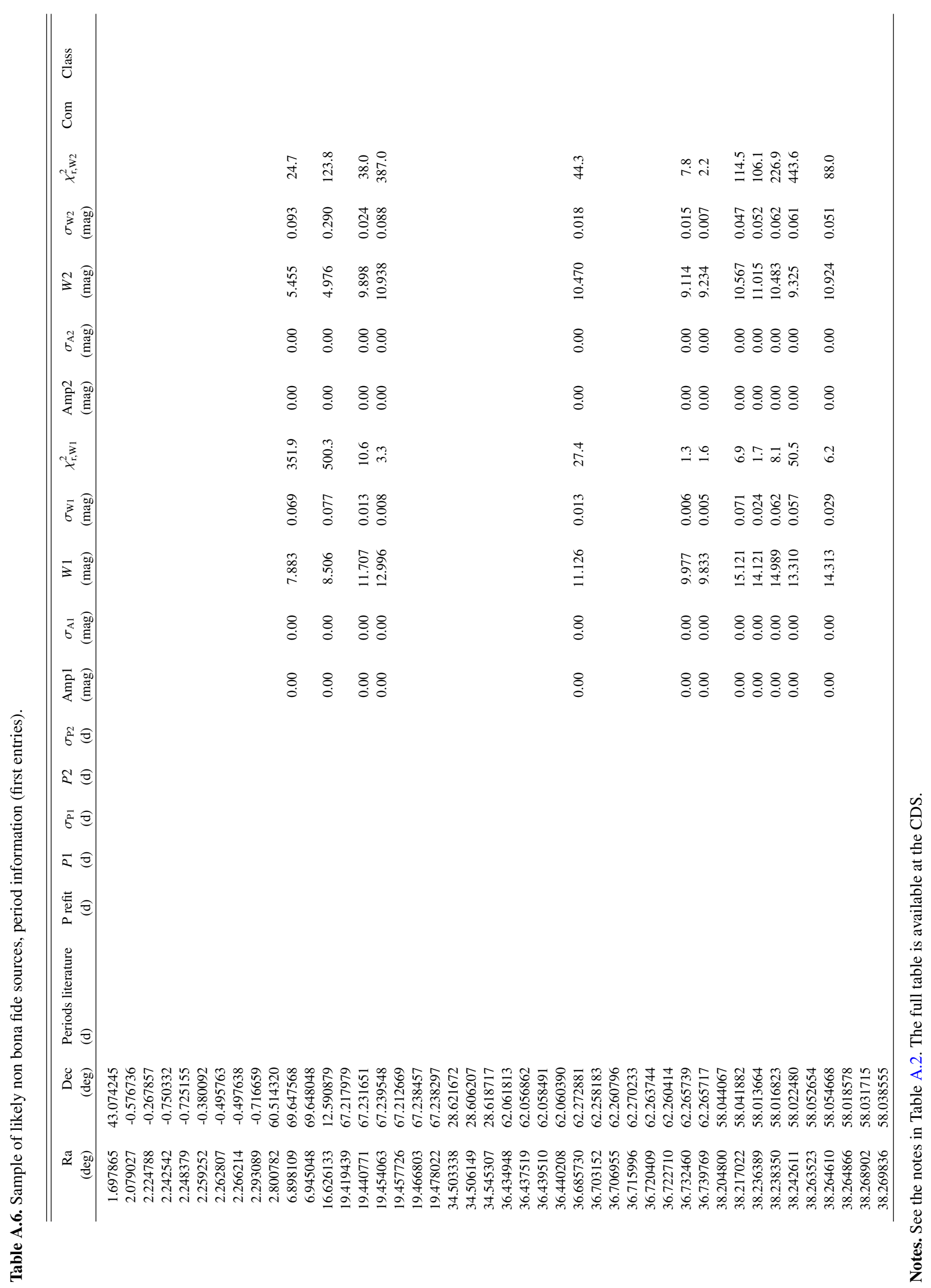




\section{Appendix B: Figures of LCs}

Figures B.1-B.11 contain the observed data and fitted LCs, with one figure per separate dataset. Figure B.12 shows the LCs of some interesting sources (see Sect. 6.4).
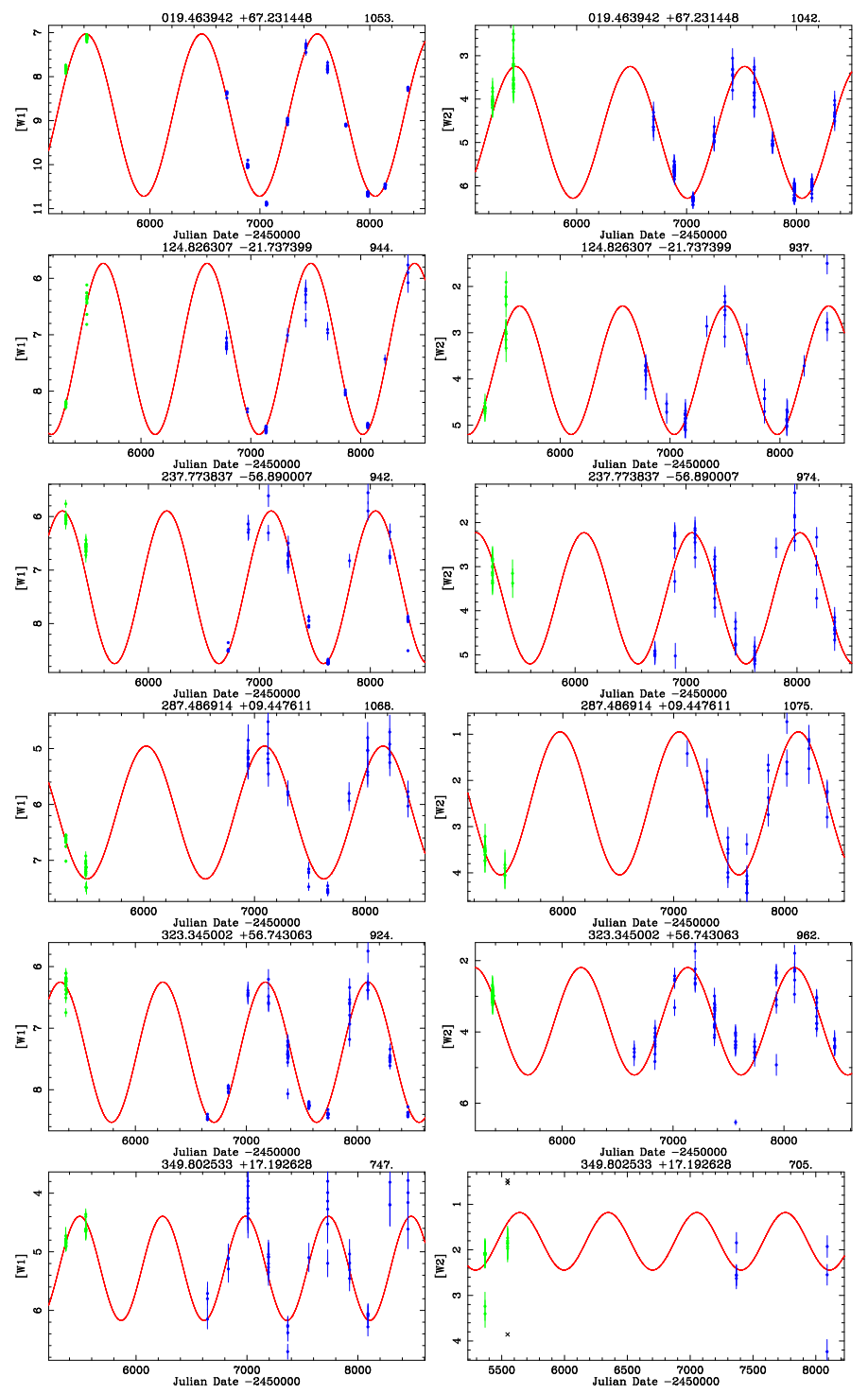

Fig. B.1. Examples of fits to WISE data (W1 on the left, W2 on the right). The identifier is listed on the top of each panel, with the period to the right. Green points refer to WISE, and blue points to NEOWISE data. Crosses indicate points excluded from the fitting. The complete set of LCs is available at https://doi .org/10.5281/zenodo. 5825878 .
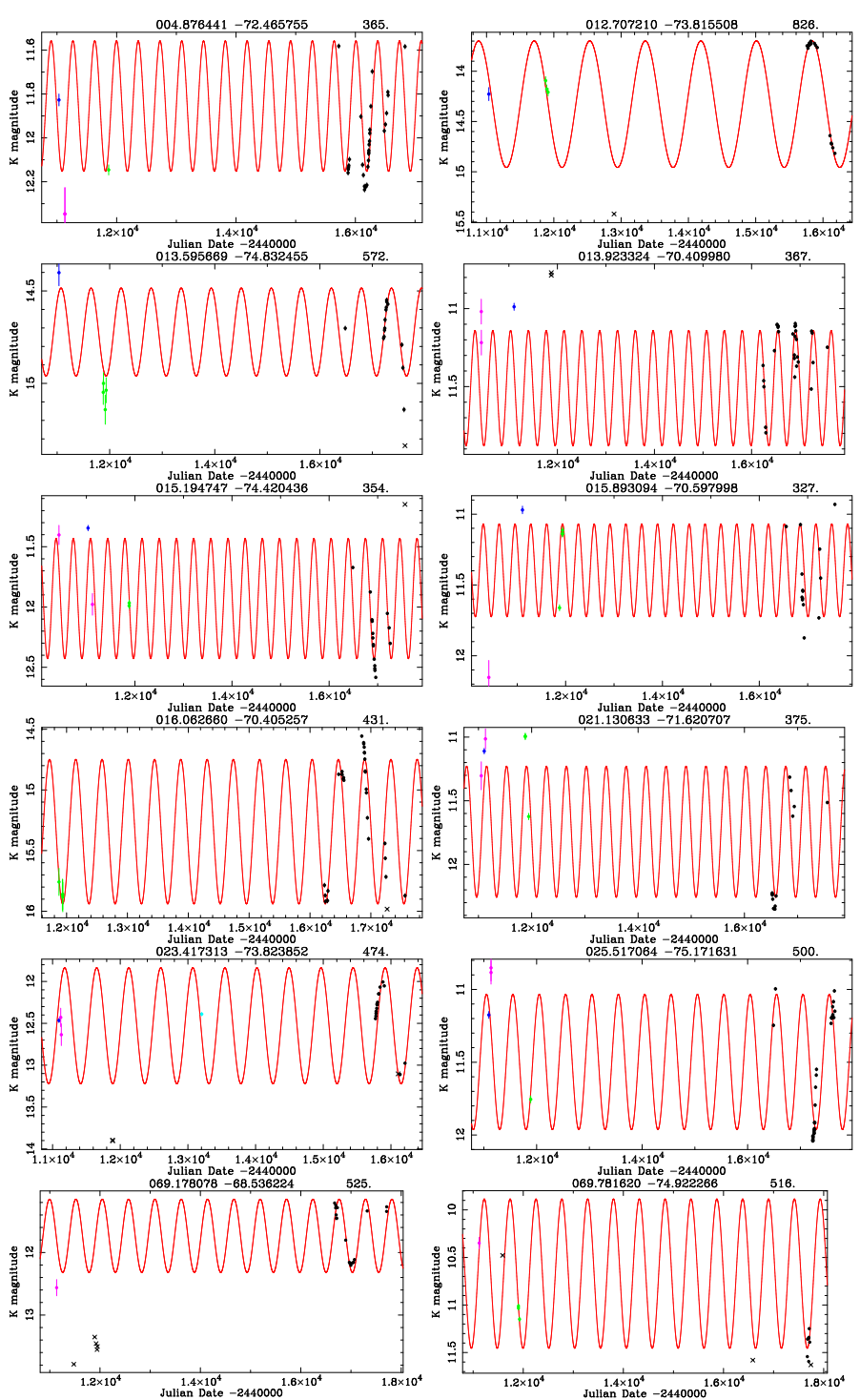

Fig. B.2. Examples of fits to VMC data $K$-band data. The identifier is listed on the top of each panel, with the period to the right. Data points (with error bars) in black show the VMC, green points represent 2MASS, dark blue points are for 2MASS-6X, light blue points are for the IRSF, and magenta corresponds to DENIS (see Groenewegen et al. 2020). 
A\&A 659, A145 (2022)
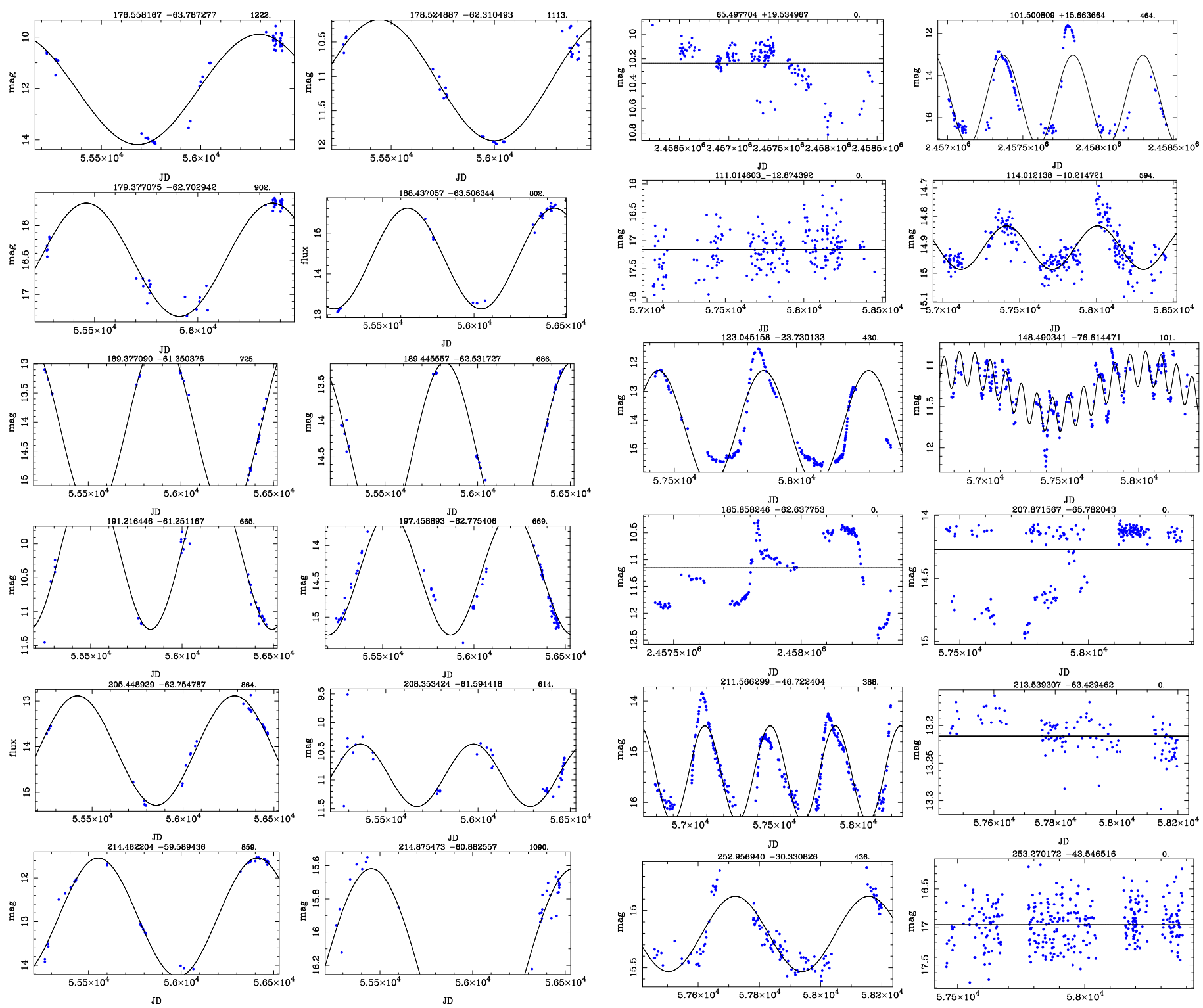

Fig. B.3. Examples of fits to VVV $K$-band data. The identifier is listed on the top of each panel, with the period to the right.
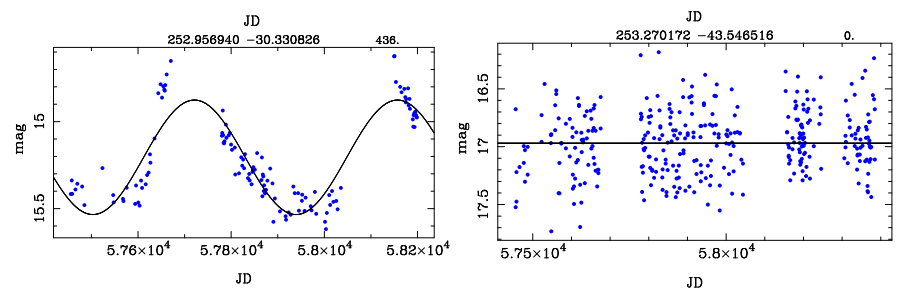

Fig. B.4. Examples of fits to ASAS-SN $V$-band data. The identifier is listed on the top of each panel, with the period to the right. 
M. A. T. Groenewegen: A WISE view on extreme AGB stars
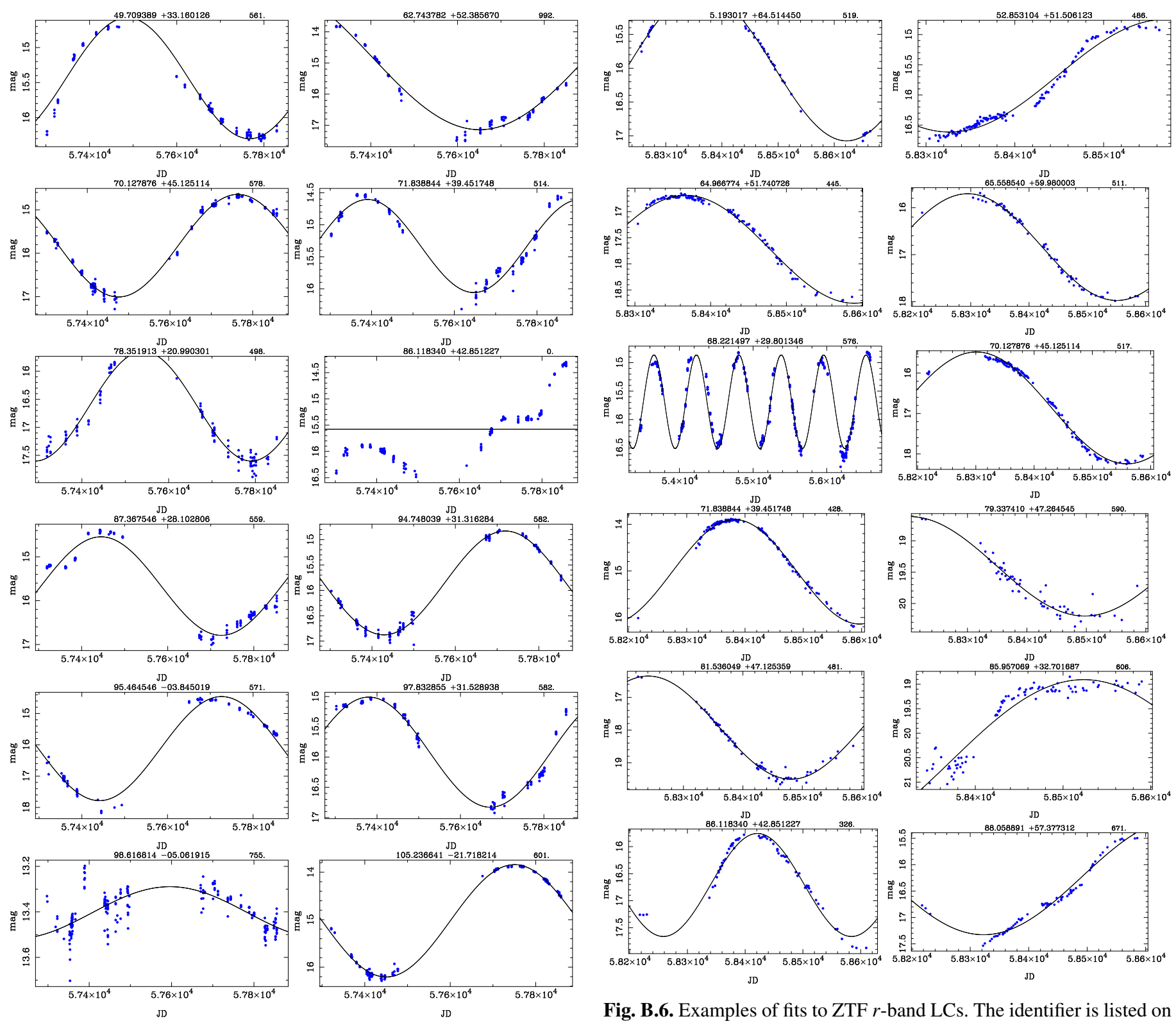

Fig. B.5. Examples of fits to ATLAS $o$-band data. The identifier is listed on the top of each panel, with the period to the right.
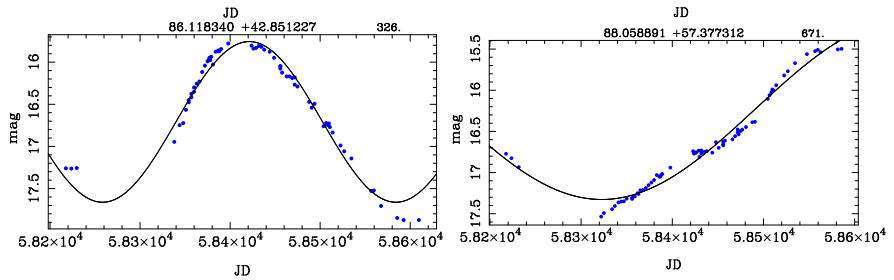

Fig. B.6. Examples of fits to ZTF $r$-band LCs. The identifier is listed on the top of each panel, with the period to the right. 

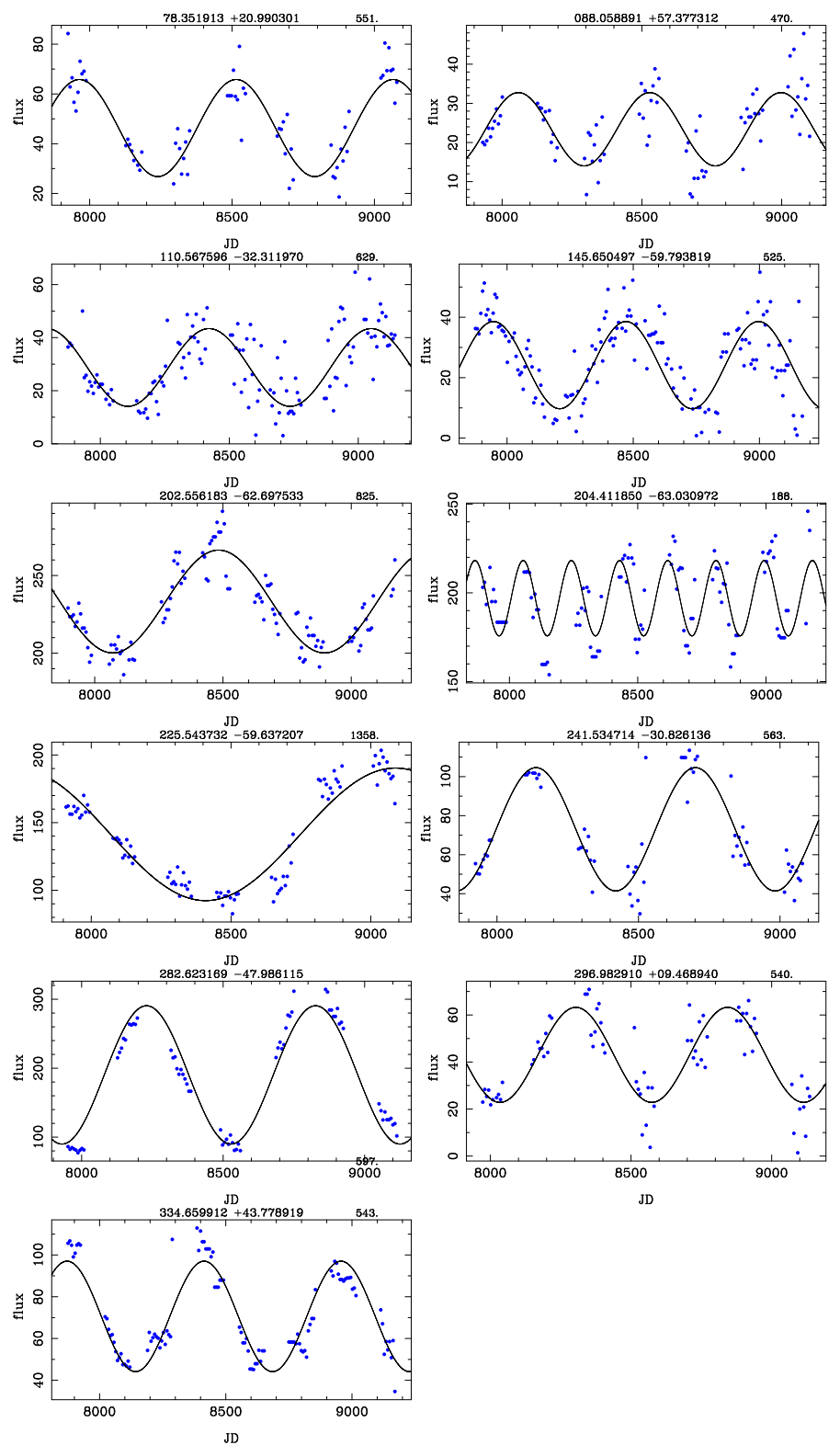

Fig. B.7. Fits to DIRBE $4.9 \mu \mathrm{m}$ flux (in Jy). The identifier is listed on top of each panel, with the period to the right.

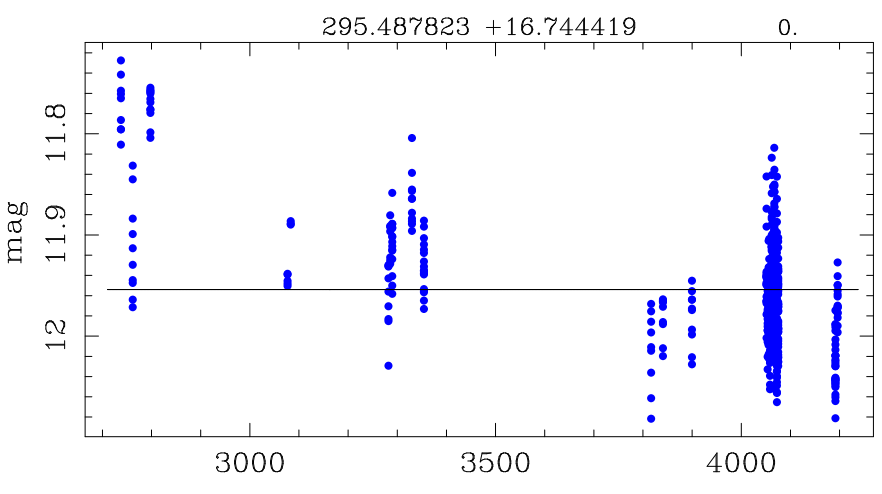

JD

Fig. B.8. Fit to one OMC source in the $V$-band. The identifier is listed on top of each panel, with the period to the right.
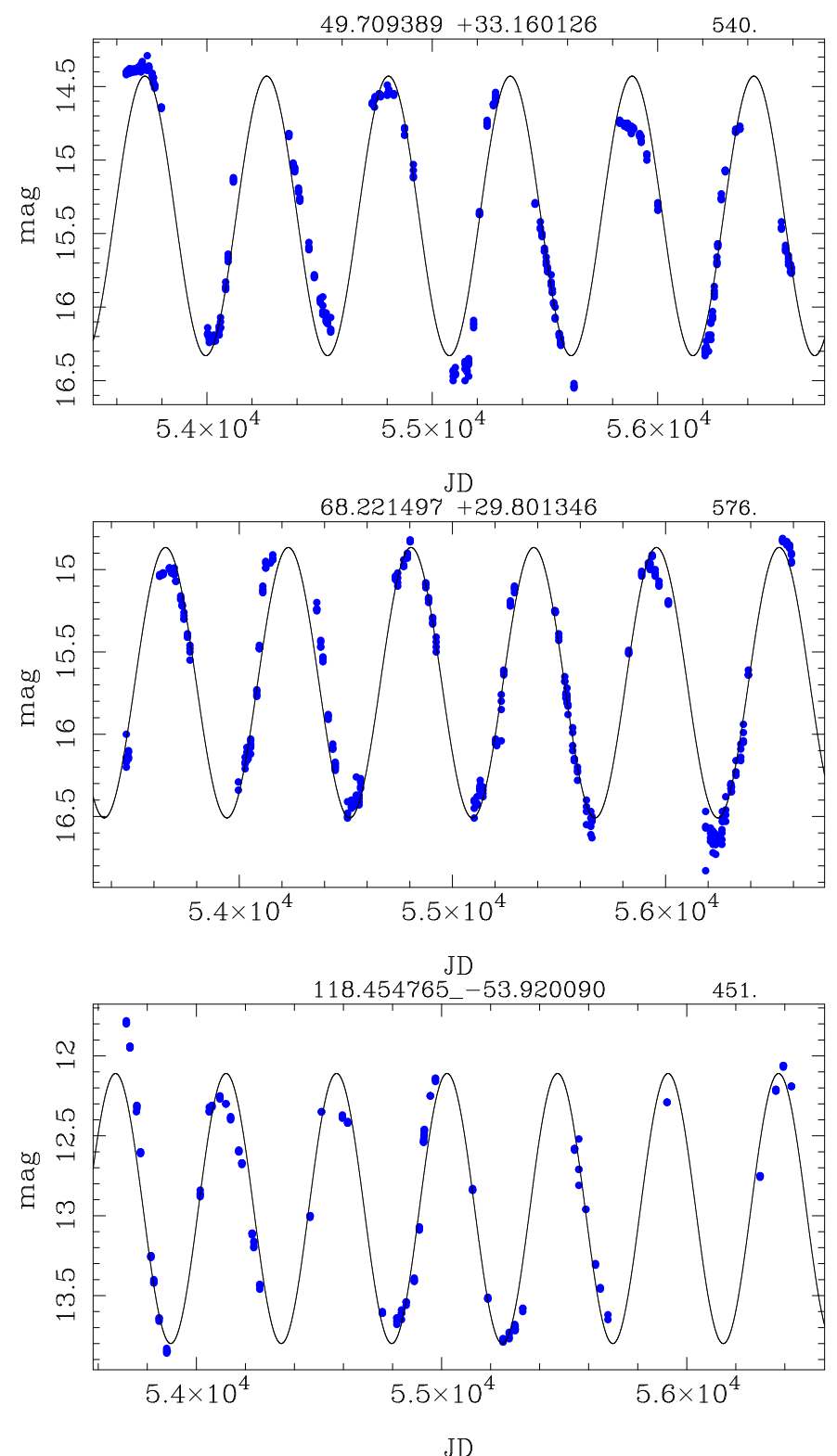

Fig. B.9. Fits to three CSS sources in the $V$-band. The identifier is listed on top of each panel, with the period to the right. 
M. A. T. Groenewegen: A WISE view on extreme AGB stars
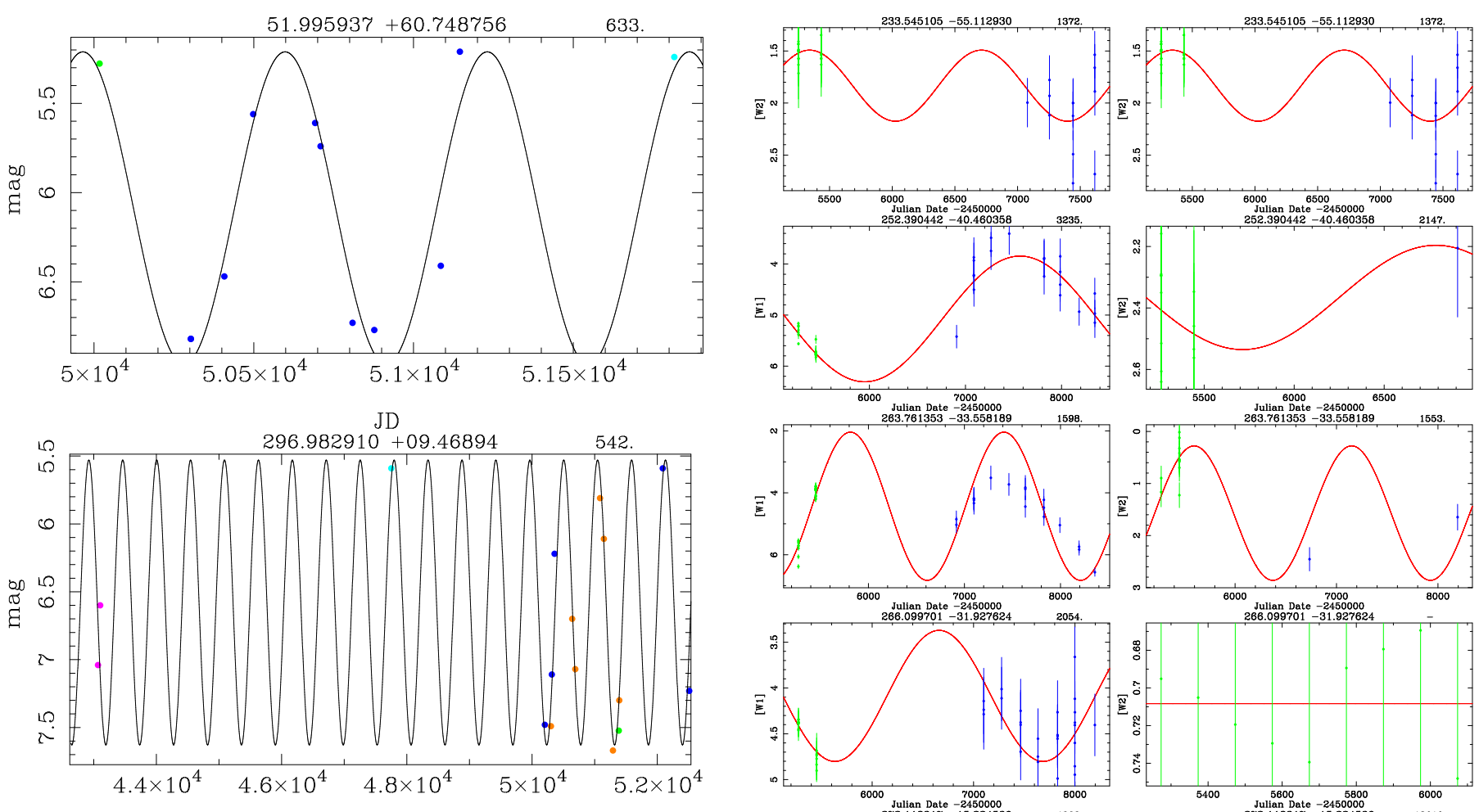

JD

Fig. B.10. Fits to $K$-band data from Kerschbaum et al. (2006). The identifier is listed on top of each panel, with the period to the right.
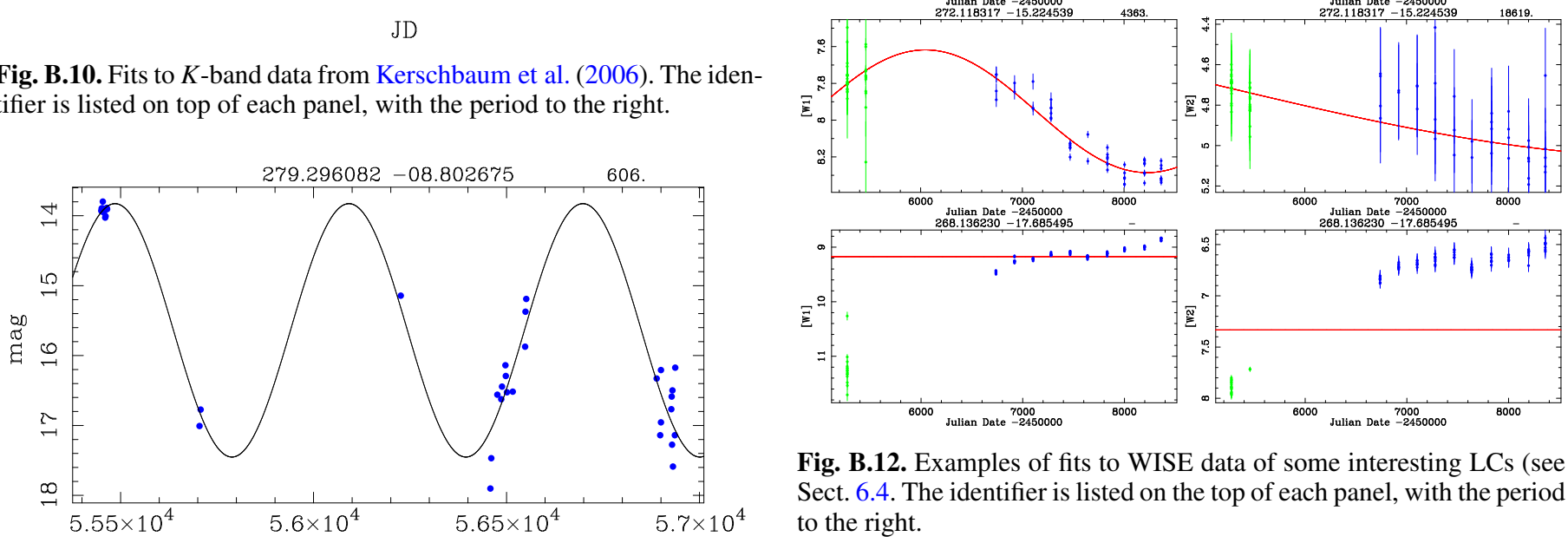

Fig. B.12. Examples of fits to WISE data of some interesting LCs (see Sect. 6.4. The identifier is listed on the top of each panel, with the period to the right.

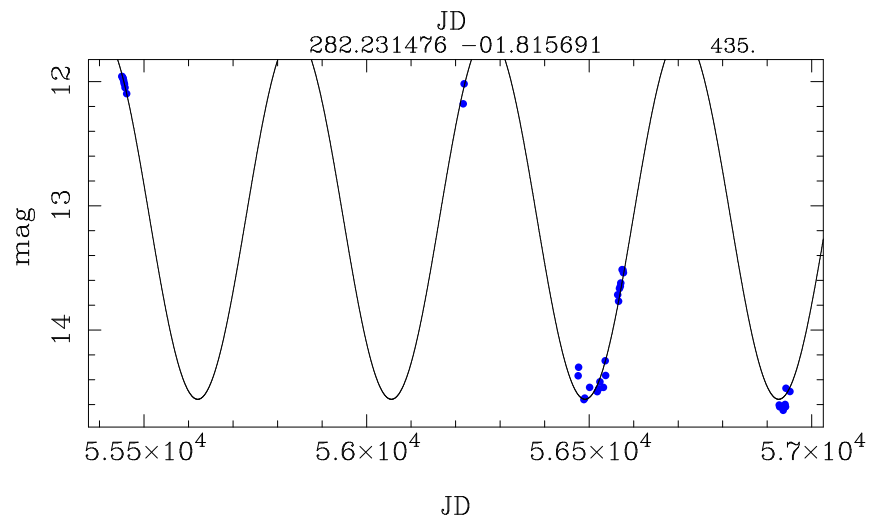

Fig. B.11. Fits to two GDS sources in the $I$-band. The identifier is listed on top of each panel, with the period to the right. 


\section{Appendix C: Constructing and fitting spectral energy distributions}

This appendix describes the construction and fitting of the spectral energy distributions (SEDs) of a subset of stars in order to find new C-rich EROs and derive their MLRs. As described in the main text, the intial subsample consisted of stars with $W 2-W 3>3.0$ based on the colours of the known C-rich EROs in Table 1. Sixty-seven stars with a blue and red detection on the $\mathrm{OH}$ maser database of Engels \& Bunzel (2015) were removed as these are confirmed O-rich sources.

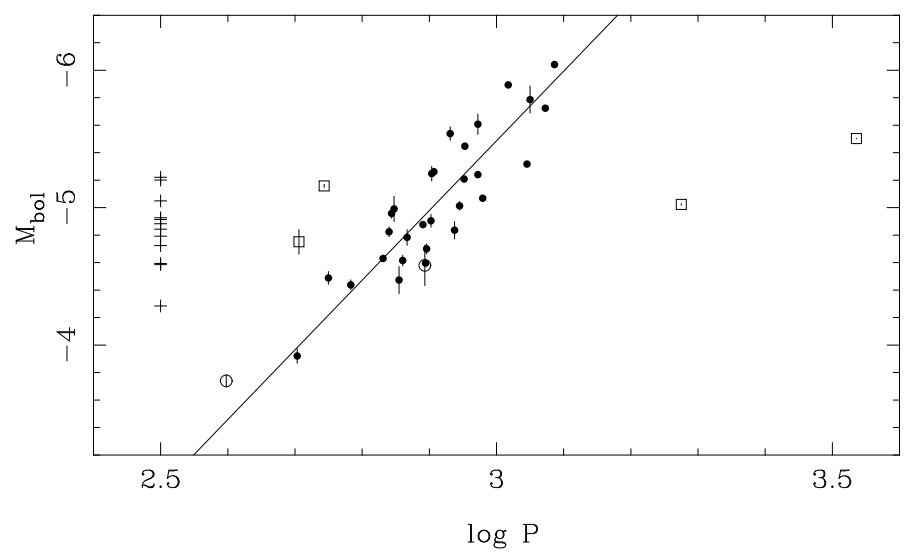

Fig. C.1. Bolometric $P L$ relation for red C-rich objects in the Magellanic Clouds. Stars without a period are plotted as plus signs at $\log P=2.5$. Stars that are excluded from the fit are plotted as open symbols. Circles (two objects) represent stars in the SMC, and the squares are objects in the LMC.

For the remaining sample of 316 objects, the following databases were queried to collect photometric data: AllWISE (Cutri 2014), Akari FIS (Yamamura et al. 2010), Akari IRC (Ishihara et al. 2010), Akari NIR PSC (Kato et al. 2012), IRAC and MIPS observations from the SAGE program ${ }^{17}$ (Meixner et al. 2006) and Gruendl \& Chu (2009), GLIMPSE (Spitzer Science Center 2009), MIPSGAL (Gutermuth \& Heyer 2015), MSX (Egan et al. 2003), IRAS Point and Faint Source Catalogue (Joint IRAS Science Working Group 1986; Moshir 1990), Herschel PACS (Herschel Point Source Catalogue Working Group 2017), Herschel PACS and SPIRE observations from the Heritage (Seale et al. 2014) and the Hi-GAL programme (Molinari et al. 2016; Elia et al. 2017), VVV (VISTA Variable in the Via Lactea Survey, datarelease 2; Minniti et al. 2017), VMC (VISTA Magellanic Survey, datarelease 4; Cioni et al. 2011), the Large Magellanic Cloud Near-Infrared Synoptic Survey (Macri et al. 2015), the IRSF Magellanic Clouds Point Source Catalogue (Kato et al. 2007), the JCMT Plane Survey (Eden et al. 2017), and ATLASGAL (Csengeri et al. 2014). After inspecting the SEDs and an initial round of model fitting (see below) the literature was searched for additional photometric data for selected sources. Details on the SEDs of individual sources are available upon reasonable request to the author.

In addition, MIR spectra were collected from the IRAS LRS (Volk \& Cohen 1989) ${ }^{18}$, the Infrared Space Observatory shortwavelength spectrograph (SWS) from Sloan et al. $(2003)^{19}$, and

\footnotetext{
17 Vizier catalogue II/305/archive for IRAC data and http://irsa. ipac. caltech.edu/applications/Gator/for MIPS data.

18 http://isc83.astro.unc.edu/iraslrs/getlrs_test.html

19 https://users.physics. unc.edu/ gcsloan/library/2003/ swsatlas.html
}
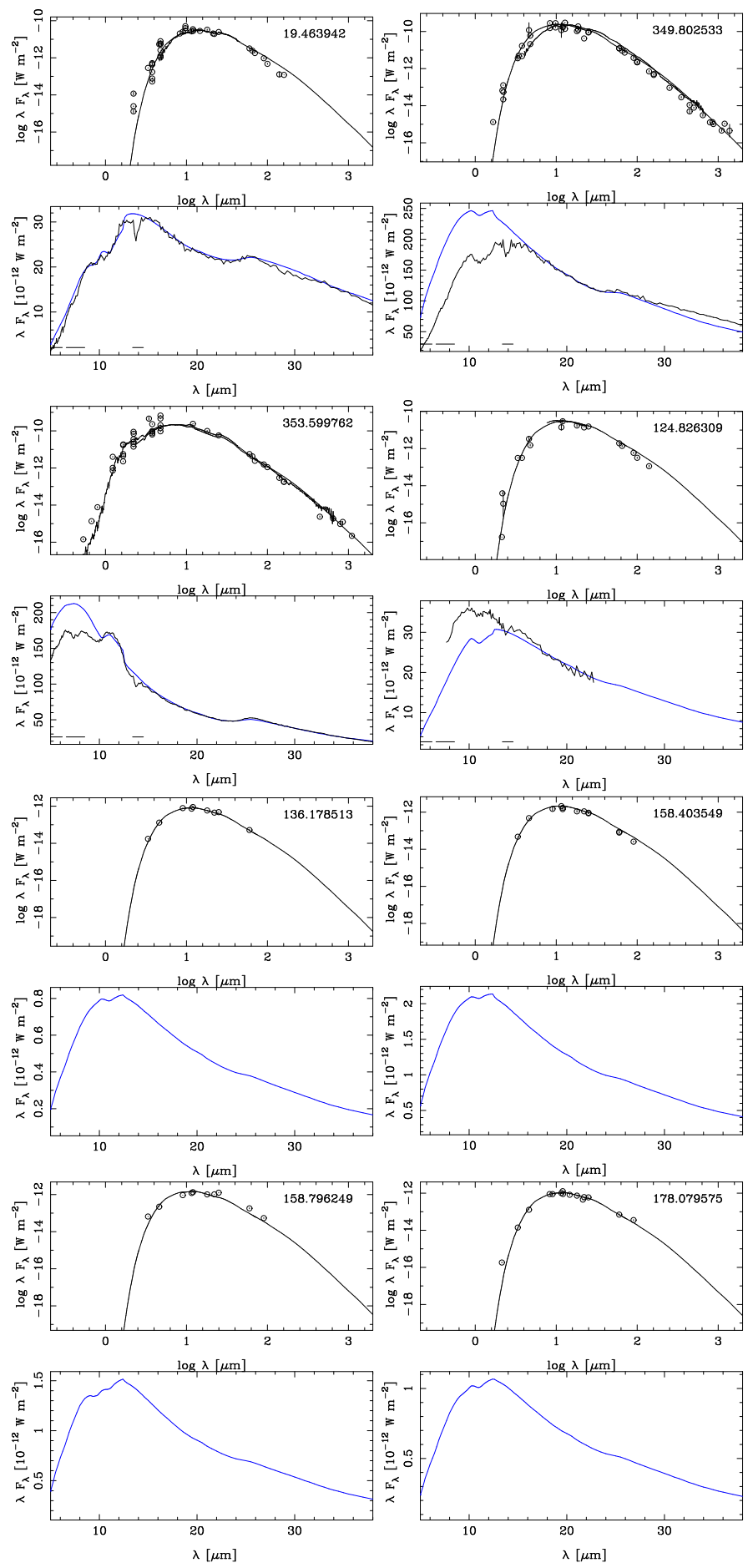

Fig. C.2. Examples of fits to the SEDs and MIR spectra of some Cstars. The complete set of SED fits for the O- and C-stars is available at https://doi .org/10.5281/zenodo. 5825878. To highlight the difference in the dust features, the observations were scaled to the models based on the average flux in the 16-20 $\mu \mathrm{m}$ region for the C-stars and the $13-15 \mu \mathrm{m}$ region for the O-stars in the MIR panels.

the SST IRS. In the latter case the spectra were retrieved through the CASSIS $^{20}$ service (Lebouteiller et al. 2011).

The SEDs and MIR spectra were modelled with MoD (Groenewegen 2012). The central stars of C-stars are represented by model atmospheres from Aringer et al. (2009), and those

${ }^{20}$ http://cassis.astro. cornell.edu 
Table C.1. Fit results of the O-star sample (first entries).

\begin{tabular}{|c|c|c|c|c|c|c|c|c|c|c|c|c|}
\hline $\begin{array}{r}\text { RA } \\
\text { (deg) }\end{array}$ & $\begin{array}{r}\text { Dec } \\
\text { (deg) }\end{array}$ & $\begin{array}{r}\text { Period } \\
\text { (days) }\end{array}$ & $\begin{array}{r}d \\
(\mathrm{kpc})\end{array}$ & $\begin{array}{r}A_{\mathrm{V}} \\
(\mathrm{mag})\end{array}$ & $\begin{array}{l}T_{\text {eff }} \\
(\mathrm{K}) \\
\end{array}$ & $\begin{array}{r}L \\
\left(L_{\odot}\right)\end{array}$ & $\tau_{0.5}$ & $\begin{array}{r}T_{\mathrm{c}} \\
(\mathrm{K}) \\
\end{array}$ & & $p$ & $\mathrm{f}$ & $\begin{array}{r}\dot{M} \\
\left(M_{\odot} \mathrm{yr}^{-1}\right)\end{array}$ \\
\hline 32. & .767200 & - & & & 720 & $26+341$ & \pm 2.1 & $2 \pm 16$ & & & & E-04 \\
\hline & & 664 & & & & & & & & & 0 & \\
\hline 84.930687 & -69.647270 & & 50.00 & & 10000 & $24 \mathrm{E} 3 \pm 132 \mathrm{E} 3$ & & & & & & \\
\hline & & - & & & & & & & 1 & & & $5 E-04$ \\
\hline & -41.122845 & 1977 & 26.94 & & & & & $1200 \pm 0$ & 0 & & 0 & E-03 \\
\hline 130.866959 & -46.111153 & - & 2.00 & & & & & & 1 & & 0 & E-05 \\
\hline & 235 & - & & & & & & & 1 & & 1 & \\
\hline & & - & & & & & & & 1 & & 1 & E-04 \\
\hline & & 936 & 4.25 & & & & & $1000 \pm 0$ & 0 & & 0 & E-03 \\
\hline & & - & 2.00 & & & & & & 1 & & 0 & \\
\hline & & - & $2 .($ & & & & & & 1 & & 1 & \\
\hline & & - & & & & & & & 0 & & 0 & \\
\hline & & - & & & & & & & 0 & & 0 & \\
\hline & & - & & & & & & & 1 & & 0 & -04 \\
\hline & & & & & & & & & 0 & & 0 & \\
\hline & & & & & & & & & 1 & & 0 & \\
\hline & & & & & & & & & 1 & & 0 & \\
\hline & & & & & & & & & 0 & & 0 & \\
\hline & 4200 & - & 2.0 & 2.5 & & & & & 1 & & 0 & \\
\hline & & - & 2.0 & & & & & & 0 & & 0 & \\
\hline & & - & & & & & & & 1 & & 0 & \\
\hline & & - & & & & & & & 0 & & 0 & \\
\hline & & 797 & 11.8 & & & & & & 1 & & 0 & \\
\hline & & & & & & & & & 0 & & 0 & \\
\hline & & & & & & & & & 1 & & & \\
\hline & & 842 & & & & & & & 0 & & 0 & \\
\hline & & & & & & & & & 1 & & 0 & -03 \\
\hline & -60.6 & - & & & & & & & 1 & & 0 & \\
\hline & & - & & & & & & & 1 & & 1 & \\
\hline & & - & 2.00 & 1.29 & & & & & 1 & & 0 & -04 \\
\hline & & - & & & & & & & 1 & & 1 & $E-04$ \\
\hline & & - & & & & & & & 1 & & 0 & $\mathrm{E}-02$ \\
\hline & & & & & & & & & 0 & & 0 & \\
\hline & & & & & & & & & 1 & & & \\
\hline & -48.687710 & 1122 & & & & & & $1000 \pm 0$ & 0 & & 0 & $8 \mathrm{E}-04$ \\
\hline & -39.095798 & - & 2. & & & & & $433 \pm 40$ & 1 & & 0 & E-04 \\
\hline & -44.92 & - & 2.0 & & & & & $1211 \pm 68$ & 1 & & 0 & E-05 \\
\hline & & - & 2.00 & & 12500 & & & & & & 0 & \\
\hline 248.374420 & -48.059303 & - & 2.00 & 1.60 & & & & $279 \pm 9$ & 1 & & 1 & $0.265 \mathrm{E}-04$ \\
\hline & -48.056137 & - & 2.00 & & 12500 & & & $237 \pm 6$ & & & 0 & $0.188 \mathrm{E}-04$ \\
\hline 249.180099 & -47.524422 & - & 2.00 & 1.38 & 7600 & $68829 \pm 7556$ & $124 \pm 6.6$ & $209 \pm 9$ & & $2.4 \pm 0.1$ & 1 & $0.451 \mathrm{E}-02$ \\
\hline
\end{tabular}

Notes. The meaning of the columns is as in Table 2. The entries are listed in order of RA. The full table is available at the CDS.

for O-stars with MARCS model atmospheres (Gustafsson et al. 2008). For stars hotter than AGB stars PHOENIX model atmospheres $^{21}$ (Hauschildt et al. 1999) were used.

For C-stars, the dust composition is a mixture of amorphous carbon (AmC), silicon carbide ( $\mathrm{SiC})$, and magnesium sulfide $(\mathrm{MgS})$. For O-stars, the dust composition is a mixture of amorphous silicates and metallic iron. When MIR spectra are available, the distinction between $\mathrm{C}$-rich and O-rich chemistry is in most cases clear and the ratio of $\mathrm{SiC} / \mathrm{AmC}$ and $\mathrm{MgS} / \mathrm{AmC}$ is estimated by eye. If no MIR spectra are available, typical ratios $(\mathrm{SiC} / \mathrm{AMC}=0.05, \mathrm{MgS} / \mathrm{AMC}=0.10)$ are adopted in the fitting.

\footnotetext{
${ }^{21}$ http://phoenix.ens-lyon.fr/Grids/BT-NextGen/ SPECTRA/
}

For the O-rich sources, a ratio of metallic iron to amorphous silicates of 0.15 is adopted, which seems to be a reasonable fit in most cases. The actual dust composition may be more complicated, but it is not our aim to study this in detail here. The distinction between $\mathrm{C}$ - and O-rich chemistry is then made by fitting the photometry alone, and adopting the best fit.

For a given set of photometry and spectra as input data, MoD determines the best-fitting luminosity (for a given distance), dust optical depth, dust temperature at the inner radius, and slope of the density profile. Any of these parameters can also be fixed.

Canonical distances to the LMC of $50 \mathrm{kpc}$ and $61 \mathrm{kpc}$ to the SMC were adopted, which are well within the error bars of the current best estimates (de Grijs et al. 2014; de Grijs \& Bono 2015). A priori, the distances to the objects in our Galaxy are unknown. However, we make use of the fact that LPVs are 
expected to follow a $P L$ relation, and that many ERO candidates in the sample are located in the LMC (and a few in the SMC). Figure C. 1 shows the derived $P L$ relation based on 31 objects and with an rms of $0.31 \mathrm{mag}$. The functional form is given in the main text, Eq. (2).

This $P L$ relation was then applied to the ERO candidates in the Galaxy which have a period, to obtain a luminosity, for both $\mathrm{C}$ - and O-rich sources. For C-rich ERO candidates in the Galaxy without a period a luminosity of $7100 L_{\odot}$ was adopted, which is the median luminosity of C-rich sources in the MCs. O-rich ERO candidates in the Galaxy without a period were placed at an arbitrary distance of $2 \mathrm{kpc}$. For a few sources with exceptionally long (and or uncertain periods) the $P L$ relation is not applied ${ }^{22}$ as it would lead to unrealistically large luminosities incompatible with an AGB status, or, for a few sources at low galactic latitudes, it would lead to large distances with correspondingly large reddenings that are incompatible with the SED. These sources were treated as if no period were available, that is $L=7100 L_{\odot}$ was assumed if it is C-rich, and a distance of $2.0 \mathrm{kpc}$ if it was O-rich. In these cases, the objects may indeed not be LPVs, or the periodicity is not related to pulsation, or the period analysis has led to a spurious result.

Interstellar reddening also needs to be taken into account in the fitting. For the sources in the MCs the recent average values from Skowron et al. (2021) were adopted, that is $A_{\mathrm{V}}=0.22$ and $0.10 \mathrm{mag}$ for LMC and SMC, respectively, adopting $A_{\mathrm{V}}=$ $3.1 E(B-V)$ and $E(B-V)=E(V-I) / 1.4$. For the Galactic sources two recent $3 \mathrm{D}$ reddening models were used to estimate the reddening in the direction of the stars in the sample. The first is described in Lallement et al. (2018) ${ }^{23}$ (hereafter STILISM) and is based on Gaia, 2MASS and APOGEE-DR14 data. For a given galactic longitude, latitude and distance, the tool returns the value of $E(B-V)$ and an error, as well as the distance to which these values refer. If this distance is smaller than the input distance the returned value for the reddening is a lower limit. In these cases, a simple estimate of the reddening at the distance of the source was made. A second reddening value was queried at a distance 0.75 times the maximum distance available in the grid in that direction. Based on this, the first derivative (with an error bar) was determined and the reddening at the distance of the target estimated. Since the total reddening flattens with distance, this estimate is an (severe) overestimate if the distance to the object is (much) larger than the last available grid point in the grid. Therefore, the extrapolated reddening is limited to twice that at the last available grid point (see below).

The second reddening model is that described in Green et al. $(2019)^{24}$ and is based on Gaia DR2 data, 2MASS, and PanSTARRS 1 data. Reddening values are provided out to 'several' $\mathrm{kpc}$ for stars north of declination $-30^{\circ}$. For 36 sources in overlap and where the distance is available in the STILISM grid, the ratio of the reddening value between STILISM and the Bayestar19 models is 0.94 with quite some spread (a median absolute deviation of 0.18 ). Given this scatter, no attempt was made to scale the two reddening maps. For over a hundred sources where the reddening is available in the 'Bayestar19' map and is extrapolated based on STILISM, it was decided to limit the extrapolated reddening to twice that at the last available grid point. The reddening model of Green et al. (2019) is the preferred one as it extends to larger distances, and it is available for 147 of the Galactic sources. The values from STILISM were adopted for the remaining 120 Galactic sources (with the linear extrapolation of the reddening and the limit to it as just described).

The results of fitting the SEDs and MIR spectra are shown is several Tables and Figures. Figure C. 2 shows some examples of fits for the C-stars in cases when there is, or not, an MIR spectrum available. The complete set of SED fits for the O- and C-stars is available at https://doi.org/10.5281/zenodo. 5825878. Tables 2 and C. 1 show the result of the fitting. The results for the $\mathrm{C}$-stars are discussed in detail in Sect. 6.5 in connection with the mass return of C-rich EROs in the solar neighbourhood. For another distance, $L \sim d^{2}$ and $\dot{M} \sim d$ to first order (ignoring the dependence of the reddening on distance). A cautionary note is made that the MLRs quoted in the last column assume spherical symmetry. The SEDs of many of the O-rich sources cannot be fitted very well and are incompatible with the spherical model rendering the MLRs estimates highly uncertain.

\footnotetext{
22 These sources are identified with $\mathrm{ra}=85.435890,294.898102$, $274.757111,256.972198,130.866959$, and 270.724915 in this paper.

${ }^{23}$ https: //stilism.obspm. fr/ (version 4.1).
}

\footnotetext{
${ }^{24}$ https://argonaut. skymaps. info The 'Bayestar19' dataset.
} 
M. A. T. Groenewegen: A WISE view on extreme AGB stars

\section{Appendix D: SEDs of P-AGB configurations}

Figure D.1 is the same as figure 6, but in the case the central star has an effective temperature of $3800 \mathrm{~K}$.
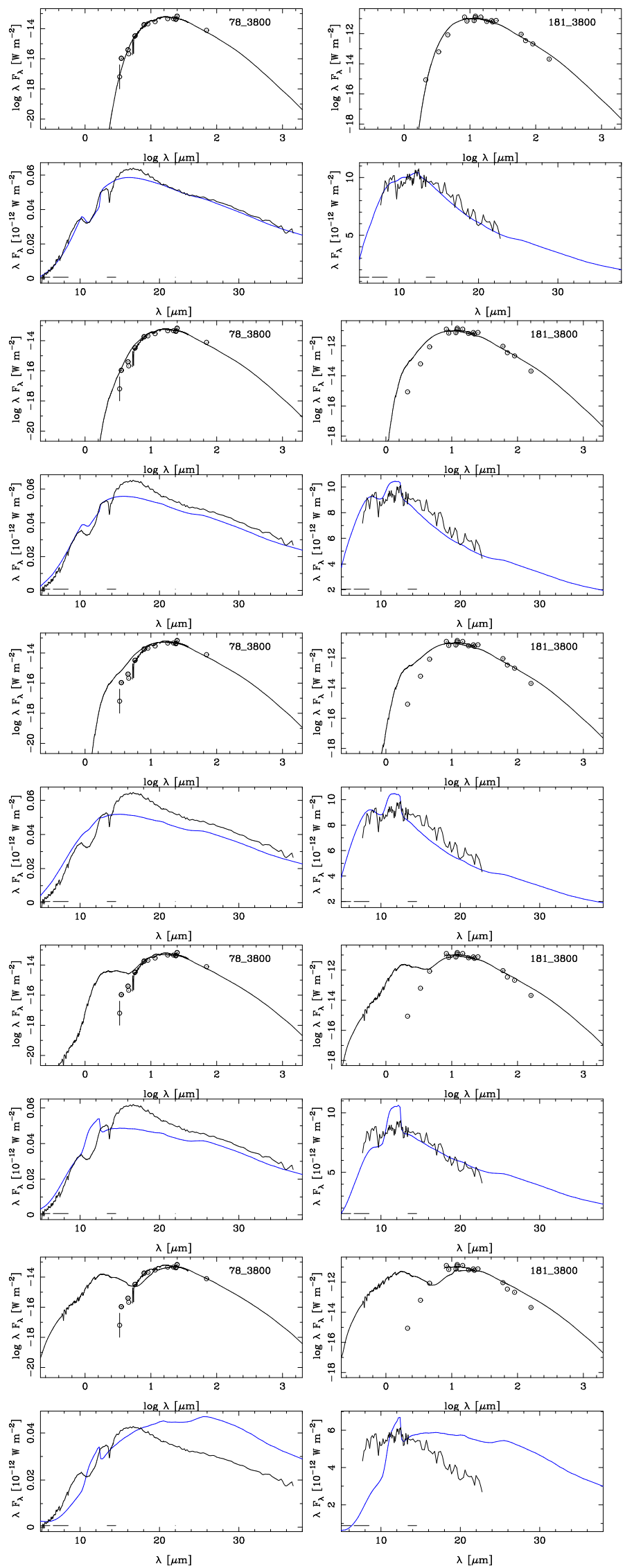

Fig. D.1. Same as for figure 6, but when the effective temperature of the central star is $3800 \mathrm{~K}$. 


\section{Appendix E: Comparison to ZTF}

The referee pointed out the paper of Chen et al. (2020), who classified about 780000 periodic variables into 11 classes using ZTF datarelease 2 data. The sample of 1992 objects was correlated with this database using a search radius of $1^{\prime \prime}$. Sixty-three matches were found, with 50 stars being classified as Miras and 12 as semi-regulars by Chen et al. (2020). Table E.1 compiles the periods found from WISE and our analysis of the ZTF data, as well as the periods found by Chen et al. (2020). We note that periods found in the literature or from refitting other datasets in the present paper are not repeated in the Table.
The periods found by Chen et al. (2020) compare rather poorly to the periods derived in the present paper from WISE data or ZTF data. Only for 20 do the periods agree within $10 \%$. For many, the periods derived in the present paper are longer, sometimes by a factor of 2 . The reason is that the ZTF data only cover a time span of 470 days, and so, citing Chen et al. (2020), 'the periods in our catalogue are most accurate for $P<100$ days. This limitation causes problems for the periods of SRs and Miras'. The advantage of the manual fitting and visual inspection, as was done in the present paper for the external data, is that periods longer than the time span of the dataset can be assigned with confidence.

Table E.1. Comparison with Chen et al. (2020).

\begin{tabular}{|c|c|c|c|c|c|c|c|}
\hline $\begin{array}{r}\mathrm{Ra} \\
(\mathrm{deg})\end{array}$ & $\begin{array}{r}\text { Dec } \\
(\mathrm{deg})\end{array}$ & $\begin{array}{l}P_{1} \\
(\mathrm{~d})\end{array}$ & $\begin{array}{l}P_{2} \\
\text { (d) }\end{array}$ & $\begin{array}{l}P_{\mathrm{ZTF}} \\
(\mathrm{d})\end{array}$ & ZTF Id & $\begin{array}{r}\text { Period } \\
\text { (d) } \\
\end{array}$ & ZTF class \\
\hline 5.193017 & 64.514450 & 549 & 270 & $519 \pm 30$ & ZTFJ002046.32+643051.9 & 284 & SR \\
\hline 6.921023 & 69.647507 & & & $637 \pm 55$ & ZTFJ002741.12+693851.5 & 425 & Mira \\
\hline 23.869953 & 49.378334 & & & $491 \pm 11$ & ZTFJ013528.77+492242.0 & 440 & Mira \\
\hline 62.743782 & 52.385670 & 920 & 928 & & ZTFJ041058.47+522308.2 & 282 & SR \\
\hline 64.966774 & 51.740726 & 463 & 477 & $445 \pm 34$ & ZTFJ041952.04+514426.4 & 400 & Mira \\
\hline 65.558540 & 59.980003 & 573 & & $511 \pm 19$ & ZTFJ042214.10+595848.0 & 440 & Mira \\
\hline 66.168434 & 48.123432 & & & & ZTFJ042440.46+480724.0 & 794 & Mira \\
\hline 70.127876 & 45.125114 & 538 & 565 & $517 \pm 5$ & ZTFJ044030.68+450730.3 & 603 & Mira \\
\hline 71.838844 & 39.451748 & 512 & 482 & $428 \pm 71$ & ZTFJ044721.35+392706.2 & 540 & Mira \\
\hline 78.351913 & 20.990301 & & & & ZTFJ051324.44+205924.9 & 454 & Mira \\
\hline 81.536049 & 47.125359 & 585 & 466 & $480 \pm 7$ & ZTFJ052608.61+470731.2 & 295 & Mira \\
\hline 85.957069 & 32.701687 & & & $606 \pm 85$ & ZTFJ054349.66+324206.5 & 487 & Mira \\
\hline 86.118340 & 42.851227 & & & $326 \pm 4$ & ZTFJ054428.38+425104.2 & 443 & Mira \\
\hline 90.742348 & 46.461830 & 453 & 430 & $406 \pm 21$ & ZTFJ060258.13+462742.4 & 374 & Mira \\
\hline 97.397789 & 8.788045 & & & $769 \pm 75$ & ZTFJ062935.45+084716.5 & 483 & Mira \\
\hline 97.832855 & 31.528938 & 537 & 559 & $666 \pm 18$ & ZTFJ063119.87+313144.0 & 521 & Mira \\
\hline 98.616814 & -5.061915 & & & - & ZTFJ063428.06-050343.0 & 171 & SR \\
\hline 101.275536 & -8.471849 & 638 & 671 & $558 \pm 13$ & ZTFJ064506.11-082818.8 & 363 & Mira \\
\hline 101.500809 & 15.663664 & & & $552 \pm 10$ & ZTFJ064600.17+153948.9 & 385 & Mira \\
\hline 103.519295 & -4.326680 & & & $377 \pm 8$ & ZTFJ065404.65-041936.3 & 187 & SR \\
\hline 104.773621 & 3.632370 & 564 & 550 & $424 \pm 9$ & ZTFJ065905.66+033756.2 & 181 & SR \\
\hline 107.645561 & -1.190360 & 627 & 675 & $539 \pm 11$ & ZTFJ071034.93-011125.5 & 406 & Mira \\
\hline 107.780273 & -0.386771 & 623 & 577 & & ZTFJ071107.28-002312.6 & 318 & SR \\
\hline 110.524391 & -3.546792 & 399 & 362 & $380 \pm 6$ & ZTFJ072205.85-033248.4 & 367 & SR \\
\hline 114.012138 & -10.214721 & & & & ZTFJ073602.90-101253.1 & 480 & Mira \\
\hline 274.574738 & -10.595833 & 602 & 553 & $402 \pm 9$ & ZTFJ181817.92-103545.0 & 457 & Mira \\
\hline 280.056274 & -5.703139 & & & $557 \pm 10$ & ZTFJ184013.49-054211.4 & 540 & Mira \\
\hline 282.231476 & -1.815691 & 429 & 432 & $397 \pm 2$ & ZTFJ184855.50-014856.9 & 394 & Mira \\
\hline 282.813995 & 1.652694 & 524 & 480 & & ZTFJ185115.41+013909.6 & 451 & Mira \\
\hline 283.761780 & 15.786965 & 450 & 447 & $458 \pm 6$ & ZTFJ185502.79+154713.2 & 445 & Mira \\
\hline 285.819824 & 9.203329 & 496 & 468 & & ZTFJ190316.78+091211.9 & 216 & SR \\
\hline 287.820007 & 17.864273 & 445 & 422 & $422 \pm 3$ & ZTFJ191116.77+175150.7 & 440 & Mira \\
\hline 288.179382 & 5.886957 & & & - & ZTFJ191243.03+055312.9 & 169 & SR \\
\hline 288.304840 & 12.003804 & 446 & 430 & $417 \pm 12$ & ZTFJ191313.18+120013.7 & 375 & Mira \\
\hline 289.141144 & 18.381018 & 437 & 417 & $407 \pm 5$ & ZTFJ191633.90+182251.8 & 409 & Mira \\
\hline 291.094452 & 32.319031 & 560 & 540 & $520 \pm 211$ & ZTFJ192422.61+321908.4 & 578 & Mira \\
\hline 291.536896 & 23.480146 & 586 & 570 & $681 \pm 25$ & ZTFJ192608.79+232848.3 & 215 & SR \\
\hline 291.658112 & 18.266577 & 520 & 590 & $490 \pm 15$ & ZTFJ192637.94+181559.0 & 500 & Mira \\
\hline 291.916656 & 23.792528 & 612 & 589 & $455 \pm 9$ & ZTFJ192739.97+234732.3 & 517 & Mira \\
\hline
\end{tabular}


Table E.1. continued.

\begin{tabular}{|c|c|c|c|c|c|c|c|}
\hline $\begin{array}{r}\mathrm{Ra} \\
(\mathrm{deg})\end{array}$ & $\begin{array}{r}\text { Dec } \\
(\operatorname{deg})\end{array}$ & $\begin{array}{l}P_{1} \\
(\mathrm{~d})\end{array}$ & $\begin{array}{l}P_{2} \\
(d) \\
\end{array}$ & $\begin{array}{l}P_{\mathrm{ZTF}} \\
(\mathrm{d})\end{array}$ & ZTF Id & $\begin{array}{r}\text { Period } \\
\text { (d) } \\
\end{array}$ & ZTF class \\
\hline 292.580017 & 17.180479 & 694 & 651 & & ZTFJ193019.22+171049.8 & 373 & SR \\
\hline 293.548218 & 19.974556 & 441 & 427 & $421 \pm 16$ & ZTFJ193411.56+195828.1 & 427 & Mira \\
\hline 294.350159 & 20.616007 & 521 & 494 & $358 \pm 12$ & ZTFJ193723.99+203657.7 & 436 & Mira \\
\hline 295.230194 & 15.337878 & 550 & 533 & $493 \pm 19$ & ZTFJ194055.25+152015.9 & 408 & Mira \\
\hline 295.535187 & 47.382542 & 460 & 453 & $394 \pm 6$ & ZTFJ194208.42+472257.0 & 304 & Mira \\
\hline 296.269775 & 6.959763 & 455 & 408 & $399 \pm 6$ & ZTFJ194504.72+065735.4 & 385 & Mira \\
\hline 297.452881 & 35.820549 & 453 & 445 & $491 \pm 7$ & ZTFJ194948.67+354913.8 & 447 & Mira \\
\hline 297.872650 & 27.149763 & 906 & 873 & $793 \pm 122$ & ZTFJ195129.43+270858.8 & 350 & Mira \\
\hline 298.620667 & 24.372654 & & & $597 \pm 37$ & ZTFJ195428.94+242221.5 & 527 & Mira \\
\hline 299.963959 & 32.536037 & & & $408 \pm 4$ & ZTFJ195951.33+323209.8 & 408 & Mira \\
\hline 302.033997 & 31.716911 & & & $468 \pm 7$ & ZTFJ200808.16+314300.9 & 422 & Mira \\
\hline 305.475128 & 36.560566 & & & - & ZTFJ202154.04+363338.0 & 556 & Mira \\
\hline 309.914825 & 50.204674 & 462 & 502 & $442 \pm 2$ & ZTFJ203939.51+501216.4 & 457 & Mira \\
\hline 315.385437 & 38.756172 & 517 & 607 & $462 \pm 4$ & ZTFJ210132.52+384522.3 & 523 & Mira \\
\hline 315.992218 & 50.244583 & 2411 & 1523 & $321 \pm 3$ & ZTFJ210358.11+501440.2 & 318 & SR \\
\hline 317.444366 & 18.413706 & 368 & 371 & $445 \pm 6$ & ZTFJ210946.62+182449.2 & 425 & Mira \\
\hline 317.659851 & 45.979378 & 542 & 548 & $442 \pm 2$ & ZTFJ211038.36+455845.9 & 424 & Mira \\
\hline 319.133759 & 36.358849 & 476 & 541 & $624 \pm 20$ & ZTFJ211632.10+362132.0 & 460 & Mira \\
\hline 325.987213 & 58.596138 & 506 & 503 & $596 \pm 48$ & ZTFJ214356.93+583546.1 & 480 & Mira \\
\hline 334.659912 & 43.778919 & & & - & ZTFJ221838.38+434644.5 & 580 & Mira \\
\hline 339.322784 & 59.454674 & 590 & 587 & $519 \pm 28$ & ZTFJ223717.44+592717.0 & 613 & Mira \\
\hline 352.573853 & 53.883614 & 506 & 553 & $573 \pm 46$ & ZTFJ233017.75+535301.1 & 520 & Mira \\
\hline 353.614540 & 43.550311 & & & $686 \pm 21$ & ZTFJ233427.48+433300.8 & 510 & Mira \\
\hline 357.904877 & 63.010296 & 611 & 626 & $558 \pm 20$ & ZTFJ235137.16+630036.9 & 292 & SR \\
\hline
\end{tabular}

Notes. Column 1 and 2: Right ascension and declination, Column 3 and 4: Period derived in the W1 and W2 filters in the present paper, Column 5: Period derived from ZTF data in the present paper (a - means no period could be determined), Column 6: ZTF identifier, Column 7: Period from Chen et al. (2020) rounded to the nearest integer, Column 8: classification in Chen et al. (2020). 